\title{
SUPPORTING INFORMATION \\ Mechanistic Insight into pH-Dependent Luminol \\ Chemiluminescence in Aqueous Solution
}

\author{
Ling Yue*, and Yi-Tong Liu
}

\begin{abstract}
Key Laboratory for Non-Equilibrium Synthesis and Modulation of Condensed Matter, Ministry of Education, School of Chemistry, Xi'an Jiaotong University, Xi'an, Shaanxi 710049, P. R. China.
\end{abstract}




\section{CONTENTS}

Scheme S1. The acidic dissociation of luminol.

Table S1. The theoretical estimated $\mathrm{p} K a, 1$ and $\mathrm{p} K a, 2$ of luminol with complete $\Delta G$ solv in SMD model (i.e. SMD1 model).

Table S2. The theoretical estimated $\mathrm{pKa}, 1$ and $\mathrm{pKa}, 2$ of luminol only with the $\Delta G$ pol. solu. - solv.

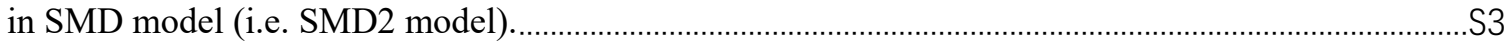

Scheme S2. The acidic dissociation of the peroxyketal (LHOOH) ........................................................S5

Table S3. The theoretical estimated $\mathrm{pKa}$ of $\mathbf{L H O O H}$ with SMD1 model. ...........................................S5

Table S4. The theoretical estimated $\mathrm{p} K a$ of LHOOH with SMD2 model. ...........................................S5

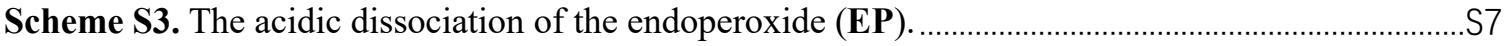

Table S5. The theoretical estimated $\mathrm{p} K a, 1$ and $\mathrm{p} K a$, 2of EP with SMD1 model..............................S7

Table S6. The theoretical estimated $\mathrm{pKa}, 1$ and $\mathrm{p} K a$, 2of EP with SMD2 model..............................S7

Scheme S4. The acidic dissociation of the endoperoxide (PI $\left.\mathbf{I}^{-}\right)$............................................................S9

Table S7. The theoretical estimated $\mathrm{pKa}$ of $\mathbf{P I}^{-}$with SMD1 model. ................................................S9

Table S8. The theoretical estimated $\mathrm{pKa}$ of $\mathbf{P I}^{-}$with SMD2 model. .................................................S9

Figure S1. The geometric structure of the optimized stationary points for the 1-C isomers...............S11

Figure S2. The geometric structure of the optimized stationary points for the 4-C isomers...............S13

Figure S3. The $S_{0}$ PECs with the SMD1 and SMD2 corrections at PBE1PBE/6-31+G*/Bondi level along the reaction path $\mathbf{L H O O H} \rightarrow \mathrm{TS} 1-4 \rightarrow$ "PI" $\rightarrow$ o-phthalic anhydride (A) and reaction path $\mathbf{E P} \rightarrow \mathbf{C P} \rightarrow \mathbf{A P}(\mathrm{B})$.

Figure S4. The $\mathrm{S}_{0}$ and $\mathrm{S}_{1}$ PECs along the $\mathrm{S}_{0}$ reaction path $\mathbf{P I}^{-} \rightarrow \mathrm{TS} 1-2 \rightarrow$ "CP$" \rightarrow \mathbf{A P}$ at TDPBE1PBE/6-31+G*//PBE1PBE/6-31+G* level in gas phase.

Figure S5. The $S_{0}$ and $S_{1}$ PECs along the $S_{0}$ reaction path $\mathbf{E P} \rightarrow T S 2-3 \rightarrow$ "CP-" $\rightarrow$ AP- at TDPBE1PBE/6-31+G*//PBE1PBE/6-31+G* level in gas phase.

Figure S6. The $S_{0}$ and $S_{1}$ PECs along the $S_{0}$ reaction path $\mathbf{E P} \rightarrow T S 2-5 \rightarrow \mathbf{C P} \rightarrow \mathbf{A P}$ at TD-PBE1PBE/6$31+\mathrm{G}^{*} / / \mathrm{PBE} 1 \mathrm{PBE} / 6-31+\mathrm{G}^{*}$ level in gas phase.

The optimized Cartesian coordinates $(\AA)$ in gas phase used in pKa estimation. at PBE1PBE/631+G*, M062X/6-31+G*, CAM-B3LYP/6-31+G* and PBE0DH/6-31+G* levels, respectively. S16 Absolute free energies of $S_{0}$ state in gas phase and solvation energies of the optimized stationary points at PBE1PBE/6-31+G*, M062X/6-31+G*, CAM-B3LYP/6-31+G* and PBE0DH/6-31+G* levels, respectively (unit: Hartree). S52

The optimized Cartesian coordinates $(\AA)$ of the stationary points and the imaginary frequencies of the transition states for the decomposition of $\mathrm{L}-\mathrm{OOH}$ in gas phase at PBE1PBE/6-31+G* level.

Absolute free energies of $S_{0}$ state in gas phase and solvation energies of the optimized stationary points at PBE1PBE/6-31+G* levels. (unit: Hartree) S70 


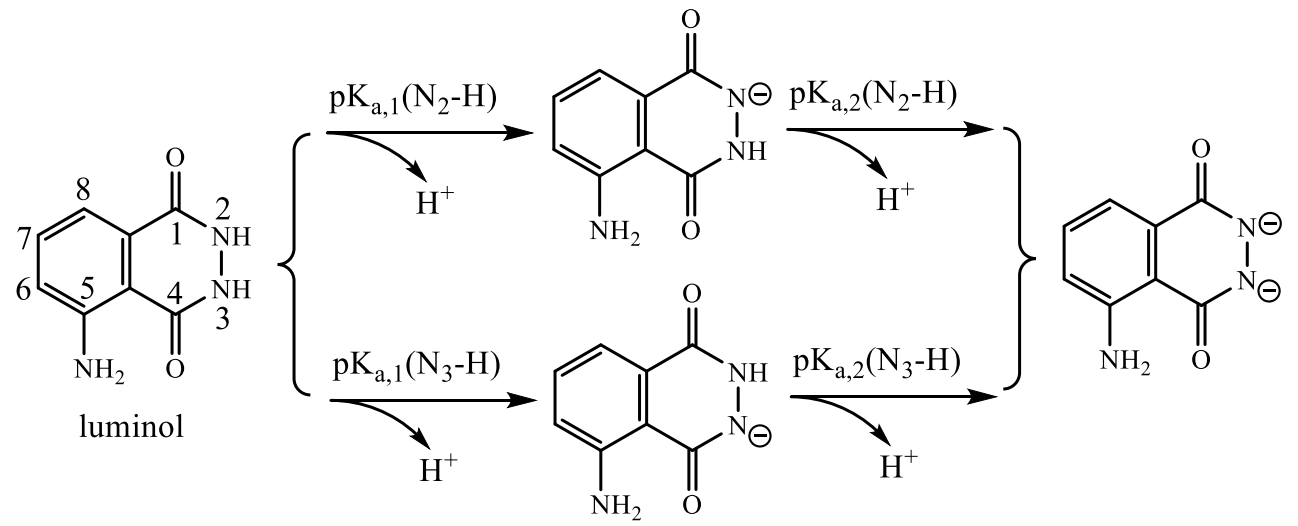

Scheme S1. The acidic dissociation of luminol.

Table S1. The theoretical estimated $\mathrm{p} K_{a, 1}$ and $\mathrm{p} K_{a, 2}$ of luminol with complete $\Delta G_{\text {solv }}$ in SMD model (i.e. SMD1 model). (The experimental values of $\mathrm{p} K_{a, 1}$ and $\mathrm{p} K_{a, 2}$ of luminol are 6.74 and $14.2 \sim 15.1,{ }^{1,2}$ respectively)

\begin{tabular}{clcccccc}
\hline Method & & UAHF & UAKS & UFF & UA0 & Bondi & Pauling \\
\hline \multirow{5}{*}{ PBE1PBE } & $\mathrm{p} K_{a, 1}\left(\mathrm{~N}_{2}-\mathrm{H}\right)$ & 18.6 & 16.8 & 9.6 & 11.1 & 8.7 & 8.7 \\
& $\mathrm{p} K_{a, 2}\left(\mathrm{~N}_{2}-\mathrm{H}\right)$ & 22.0 & 22.2 & 27.1 & 28.2 & 22.8 & 22.0 \\
& $\mathrm{p} K_{a, 1}\left(\mathrm{~N}_{3}-\mathrm{H}\right)$ & 18.5 & 16.8 & 9.2 & 10.8 & 8.7 & 8.8 \\
& $\mathrm{p} K_{a, 2}\left(\mathrm{~N}_{3}-\mathrm{H}\right)$ & 22.1 & 22.1 & 27.4 & 28.5 & 22.9 & 21.9 \\
\hline \multirow{5}{*}{ M062X } & $\mathrm{p} K_{a, 1}\left(\mathrm{~N}_{2}-\mathrm{H}\right)$ & 16.8 & 15.0 & 8.0 & 9.5 & 6.9 & 6.8 \\
& $\mathrm{p} K_{a, 2}\left(\mathrm{~N}_{2}-\mathrm{H}\right)$ & 20.2 & 20.3 & 25.5 & 26.5 & 21.0 & 20.2 \\
& $\mathrm{p} K_{a, 1}\left(\mathrm{~N}_{3}-\mathrm{H}\right)$ & 16.7 & 15.1 & 7.8 & 9.3 & 7.0 & 7.0 \\
& $\mathrm{p} K_{a, 2}\left(\mathrm{~N}_{3}-\mathrm{H}\right)$ & 20.3 & 20.1 & 25.6 & 26.7 & 21.0 & 19.9 \\
\hline \multirow{5}{*}{ CAM-B3LYP } & $\mathrm{p} K_{a, 1}\left(\mathrm{~N}_{2}-\mathrm{H}\right)$ & 17.9 & 16.1 & 8.8 & 10.3 & 8.0 & 8.0 \\
& $\mathrm{p} K_{a, 2}\left(\mathrm{~N}_{2}-\mathrm{H}\right)$ & 21.0 & 21.2 & 26.1 & 27.1 & 21.9 & 21.2 \\
& $\mathrm{p} K_{a, 1}\left(\mathrm{~N}_{3}-\mathrm{H}\right)$ & 18.0 & 16.3 & 8.7 & 10.2 & 8.1 & 8.3 \\
& $\mathrm{p} K_{a, 2}\left(\mathrm{~N}_{3}-\mathrm{H}\right)$ & 20.9 & 21.0 & 26.2 & 27.2 & 21.8 & 21.0 \\
\hline \multirow{5}{*}{ PBE0DH } & $\mathrm{p} K_{a, 1}\left(\mathrm{~N}_{2}-\mathrm{H}\right)$ & 18.6 & 16.8 & 9.6 & 11.1 & 8.8 & 8.7 \\
& $\mathrm{p} K_{a, 2}\left(\mathrm{~N}_{2}-\mathrm{H}\right)$ & 22.3 & 22.5 & 27.4 & 28.5 & 23.2 & 22.3 \\
& $\mathrm{p} K_{a, 1}\left(\mathrm{~N}_{3}-\mathrm{H}\right)$ & 18.6 & 16.9 & 9.4 & 10.9 & 8.8 & 8.8 \\
& $\mathrm{p} K_{a, 2}\left(\mathrm{~N}_{3}-\mathrm{H}\right)$ & 22.3 & 22.4 & 27.7 & 28.7 & 23.2 & 22.2 \\
\hline
\end{tabular}

Table S2. The theoretical estimated $\mathrm{p} K_{a, 1}$ and $\mathrm{p} K_{a, 2}$ of luminol only with the $\Delta G_{\text {pol.solu.solv. }}$ in SMD model (i.e. SMD2 model). (The experimental values of $\mathrm{p} K_{a, 1}$ and $\mathrm{p} K_{a, 2}$ of luminol are 6.74 and $14.2 \sim 15.1,{ }^{1,2}$ respectively)

\begin{tabular}{clcccccc}
\hline Method & & UAHF & UAKS & UFF & UA0 & Bondi & Pauling \\
\hline \multirow{5}{*}{ PBE1PBE } & $\mathrm{p} K_{a, 1}\left(\mathrm{~N}_{2}-\mathrm{H}\right)$ & 18.0 & 15.6 & 7.7 & 9.3 & 6.2 & 5.8 \\
& $\mathrm{p} K_{a, 2}\left(\mathrm{~N}_{2}-\mathrm{H}\right)$ & 13.4 & 12.9 & 22.5 & 23.3 & 15.9 & 14.4 \\
& $\mathrm{p} K_{a, 1}\left(\mathrm{~N}_{3}-\mathrm{H}\right)$ & 18.6 & 16.3 & 7.9 & 9.5 & 6.8 & 6.7 \\
& $\mathrm{p} K_{a, 2}\left(\mathrm{~N}_{3}-\mathrm{H}\right)$ & 12.9 & 12.2 & 22.3 & 23.1 & 15.2 & 13.5 \\
\hline & & & $\mathrm{S} 3$ & & & &
\end{tabular}




\begin{tabular}{clcccccc}
\hline \multirow{4}{*}{ M062X } & $\mathrm{p} K_{a, 1}\left(\mathrm{~N}_{2}-\mathrm{H}\right)$ & 16.6 & 14.4 & 6.4 & 8.0 & 4.9 & 4.8 \\
& $\mathrm{p} K_{a, 2}\left(\mathrm{~N}_{2}-\mathrm{H}\right)$ & 11.5 & 10.6 & 20.7 & 21.5 & 13.7 & 11.9 \\
& $\mathrm{p} K_{a, 1}\left(\mathrm{~N}_{3}-\mathrm{H}\right)$ & 16.1 & 13.6 & 6.0 & 7.6 & 4.2 & 3.8 \\
& $\mathrm{p} K_{a, 2}\left(\mathrm{~N}_{3}-\mathrm{H}\right)$ & 12.0 & 11.5 & 21.0 & 21.9 & 14.3 & 12.9 \\
\hline \multirow{5}{*}{ CAM-B3LYP } & $\mathrm{p} K_{a, 1}\left(\mathrm{~N}_{2}-\mathrm{H}\right)$ & 18.0 & 15.7 & 7.2 & 8.8 & 6.1 & 6.2 \\
& $\mathrm{p} K_{a, 2}\left(\mathrm{~N}_{2}-\mathrm{H}\right)$ & 12.1 & 11.5 & 21.2 & 22.0 & 14.4 & 12.9 \\
& $\mathrm{p} K_{a, 1}\left(\mathrm{~N}_{3}-\mathrm{H}\right)$ & 17.3 & 14.9 & 6.9 & 8.4 & 5.4 & 5.2 \\
& $\mathrm{p} K_{a, 2}\left(\mathrm{~N}_{3}-\mathrm{H}\right)$ & 12.8 & 12.3 & 21.5 & 22.4 & 15.1 & 13.9 \\
\hline \multirow{3}{*}{ PBE0DH } & $\mathrm{p} K_{a, 1}\left(\mathrm{~N}_{2}-\mathrm{H}\right)$ & 17.9 & 15.7 & 7.7 & 9.3 & 6.4 & 6.3 \\
& $\mathrm{p} K_{a, 2}\left(\mathrm{~N}_{2}-\mathrm{H}\right)$ & 12.9 & 12.1 & 22.4 & 23.2 & 15.3 & 13.5 \\
& $\mathrm{p} K_{a, 1}\left(\mathrm{~N}_{3}-\mathrm{H}\right)$ & 17.4 & 15.0 & 7.5 & 9.0 & 5.8 & 5.4 \\
& $\mathrm{p} K_{a, 2}\left(\mathrm{~N}_{3}-\mathrm{H}\right)$ & 13.4 & 12.8 & 22.6 & 23.4 & 15.9 & 14.4 \\
\hline
\end{tabular}




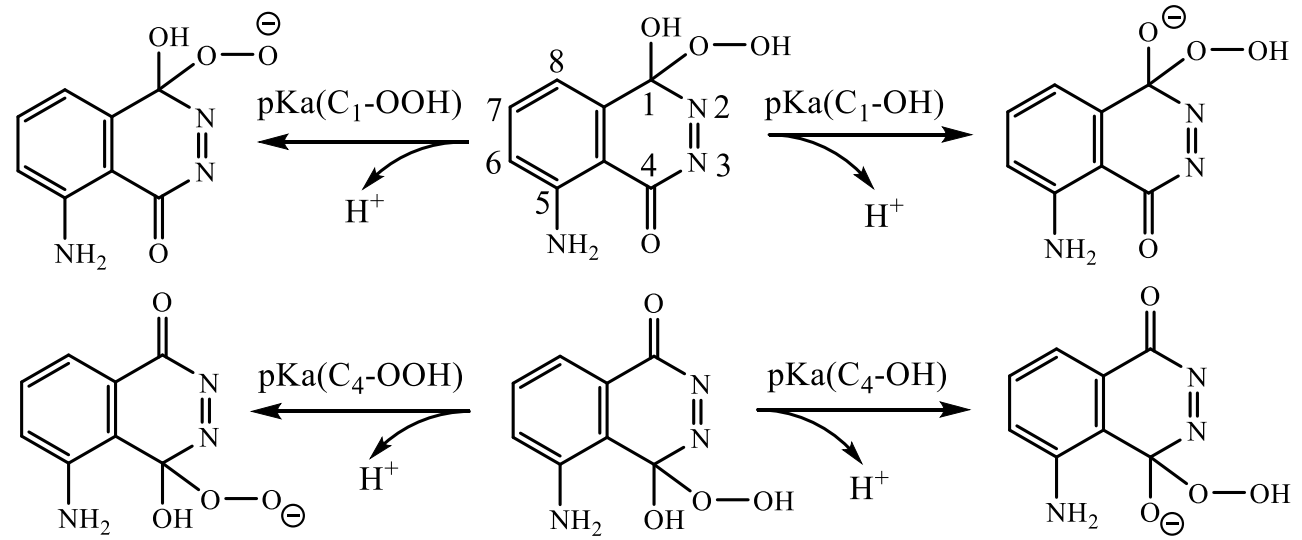

Scheme S2. The acidic dissociation of the peroxyketal (LHOOH).

Table S3. The theoretical estimated $\mathrm{p} K_{a}$ of $\mathbf{L H O O H}$ with SMD1 model.

\begin{tabular}{cccccccc}
\hline Method & & UAHF & UAKS & UFF & UA0 & Bondi & Pauling \\
\hline \multirow{5}{*}{ PBE1PBE } & $\mathrm{p} K_{a}\left(\mathrm{C}_{1}-\mathrm{OH}\right)$ & 11.7 & 11.4 & 6.6 & 9.3 & 6.1 & 5.9 \\
& $\mathrm{p} K_{a}(\mathrm{C} 1-\mathrm{OOH})$ & 22.4 & 21.6 & 18.5 & 20.2 & 17.1 & 16.8 \\
& $\mathrm{p} K_{a}\left(\mathrm{C}_{4}-\mathrm{OH}\right)$ & 10.5 & 10.5 & 4.6 & 7.4 & 4.5 & 4.5 \\
& $\mathrm{p} K_{a}\left(\mathrm{C}_{4}-\mathrm{OOH}\right)$ & 20.3 & 18.6 & 17.1 & 18.8 & 15.3 & 14.7 \\
\hline \multirow{5}{*}{ M062X } & $\mathrm{p} K_{a}\left(\mathrm{C}_{1}-\mathrm{OH}\right)$ & 12.8 & 12.6 & 7.9 & 10.6 & 7.3 & 7.0 \\
& $\mathrm{p} K_{a}(\mathrm{C} 1-\mathrm{OOH})$ & 18.9 & 17.2 & 17.3 & 19.0 & 14.4 & 13.0 \\
& $\mathrm{p} K_{a}\left(\mathrm{C}_{4}-\mathrm{OH}\right)$ & 11.0 & 11.0 & 5.4 & 8.2 & 5.4 & 5.4 \\
& $\mathrm{p} K_{a}\left(\mathrm{C}_{4}-\mathrm{OOH}\right)$ & 17.4 & 16.8 & 14.3 & 16.2 & 13.1 & 12.9 \\
\hline \multirow{5}{*}{ CAM-B3LYP } & $\mathrm{p} K_{a}\left(\mathrm{C}_{1}-\mathrm{OH}\right)$ & 10.9 & 10.7 & 5.7 & 8.4 & 5.2 & 5.1 \\
& $\mathrm{p} K_{a}(\mathrm{C} 1-\mathrm{OOH})$ & 18.6 & 16.8 & 15.9 & 17.5 & 13.6 & 12.8 \\
& $\mathrm{p} K_{a}\left(\mathrm{C}_{4}-\mathrm{OH}\right)$ & 9.9 & 9.9 & 3.8 & 6.6 & 4.0 & 4.1 \\
& $\mathrm{p} K_{a}\left(\mathrm{C}_{4}-\mathrm{OOH}\right)$ & 16.7 & 14.9 & 14.2 & 16.0 & 12.2 & 11.5 \\
\hline & $\mathrm{p} K_{a}\left(\mathrm{C}_{1}-\mathrm{OH}\right)$ & 12.4 & 12.1 & 7.5 & 10.2 & 6.9 & 6.6 \\
& $\mathrm{p} K_{a}(\mathrm{C} 1-\mathrm{OOH})$ & 22.7 & 21.6 & 19.3 & 21.0 & 17.4 & 16.7 \\
& $\mathrm{p} K_{a}\left(\mathrm{C}_{4}-\mathrm{OH}\right)$ & 11.1 & 11.1 & 5.3 & 8.1 & 5.3 & 5.3 \\
& $\mathrm{p} K_{a}\left(\mathrm{C}_{4}-\mathrm{OOH}\right)$ & 20.3 & 18.5 & 17.6 & 19.3 & 15.5 & 14.6 \\
\hline
\end{tabular}

Table S4. The theoretical estimated $\mathrm{p} K_{a}$ of LHOOH with SMD2 model.

\begin{tabular}{cccccccc}
\hline Functional & & UAHF & UAKS & UFF & UA0 & Bondi & Pauling \\
\hline \multirow{5}{*}{ PBE1PBE } & $\mathrm{p} K_{a}\left(\mathrm{C}_{1}-\mathrm{OH}\right)$ & 9.7 & 9.2 & 4.7 & 7.6 & 3.2 & 2.5 \\
& $\mathrm{p} K_{a}(\mathrm{C} 1-\mathrm{OOH})$ & 18.4 & 16.9 & 15.7 & 17.4 & 12.6 & 11.5 \\
& $\mathrm{p} K_{a}\left(\mathrm{C}_{4}-\mathrm{OH}\right)$ & 10.2 & 10.2 & 3.8 & 6.9 & 3.2 & 2.8 \\
& $\mathrm{p} K_{a}\left(\mathrm{C}_{4}-\mathrm{OOH}\right)$ & 16.9 & 14.6 & 14.7 & 16.6 & 11.3 & 9.7 \\
\hline \multirow{3}{*}{ M062X } & $\mathrm{p} K_{a}\left(\mathrm{C}_{1}-\mathrm{OH}\right)$ & 10.7 & 10.3 & 5.8 & 8.8 & 4.3 & 3.5 \\
& $\mathrm{p} K_{a}(\mathrm{C} 1-\mathrm{OOH})$ & 14.9 & 12.5 & 13.8 & 15.6 & 9.0 & 6.6 \\
& $\mathrm{p} K_{a}\left(\mathrm{C}_{4}-\mathrm{OH}\right)$ & 10.4 & 10.4 & 4.3 & 7.4 & 3.7 & 3.5
\end{tabular}




\begin{tabular}{cccccccc} 
& $\mathrm{p} K_{a}\left(\mathrm{C}_{4}-\mathrm{OOH}\right)$ & 16.1 & 15.1 & 12.8 & 14.9 & 10.5 & 9.7 \\
\hline & $\mathrm{p} K_{a}\left(\mathrm{C}_{1}-\mathrm{OH}\right)$ & 9.1 & 8.7 & 3.8 & 6.8 & 2.5 & 2.0 \\
CAM-B3LYP & $\mathrm{p} K_{a}(\mathrm{C} 1-\mathrm{OOH})$ & 14.3 & 11.9 & 12.4 & 14.2 & 8.3 & 6.5 \\
& $\mathrm{p} K_{a}\left(\mathrm{C}_{4}-\mathrm{OH}\right)$ & 9.6 & 9.6 & 2.8 & 6.0 & 2.5 & 2.4 \\
& $\mathrm{p} K_{a}\left(\mathrm{C}_{4}-\mathrm{OOH}\right)$ & 13.7 & 11.3 & 11.7 & 13.5 & 8.0 & 6.5 \\
\hline \multirow{5}{*}{ PBE0DH } & $\mathrm{p} K_{a}\left(\mathrm{C}_{1}-\mathrm{OH}\right)$ & 10.3 & 9.9 & 5.6 & 8.5 & 3.9 & 3.1 \\
& $\mathrm{p} K_{a}(\mathrm{C} 1-\mathrm{OOH})$ & 17.6 & 15.7 & 15.8 & 17.5 & 11.8 & 10.1 \\
& $\mathrm{p} K_{a}\left(\mathrm{C}_{4}-\mathrm{OH}\right)$ & 10.6 & 10.6 & 4.4 & 7.5 & 3.8 & 3.4 \\
& $\mathrm{p} K_{a}\left(\mathrm{C}_{4}-\mathrm{OOH}\right)$ & 16.6 & 14.1 & 14.8 & 16.7 & 10.9 & 9.0 \\
\hline
\end{tabular}




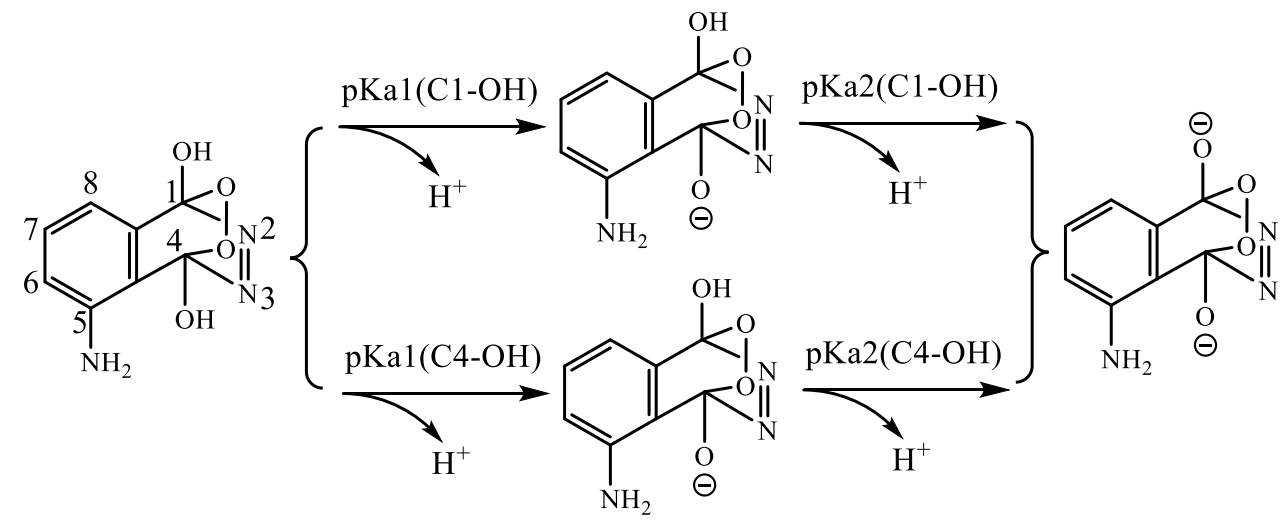

Scheme S3. The acidic dissociation of the endoperoxide (EP).

Table S5. The theoretical estimated $\mathrm{p} K_{a, 1}$ and $\mathrm{p} K_{a, 2}$ of $\mathbf{E P}$ with SMD1 model.

\begin{tabular}{clcccccc}
\hline Method & & UAHF & UAKS & UFF & UA0 & Bondi & Pauling \\
\hline \multirow{5}{*}{ PBE1PBE } & $\mathrm{p} K_{a, 1}\left(\mathrm{C}_{1}-\mathrm{OH}\right)$ & 8.9 & 8.7 & 6.8 & 8.8 & 5.7 & 5.3 \\
& $\mathrm{p} K_{a, 2}\left(\mathrm{C}_{1}-\mathrm{OH}\right)$ & 12.9 & 13.0 & 12.9 & 14.4 & 9.6 & 8.6 \\
& $\mathrm{p} K_{a, 1}\left(\mathrm{C}_{4}-\mathrm{OH}\right)$ & 8.1 & 8.2 & 5.2 & 7.3 & 4.6 & 4.4 \\
& $\mathrm{p} K_{a, 2}\left(\mathrm{C}_{4}-\mathrm{OH}\right)$ & 13.6 & 13.5 & 14.6 & 15.9 & 10.8 & 9.5 \\
\hline \multirow{5}{*}{ M062X } & $\mathrm{p} K_{a, 1}\left(\mathrm{C}_{1}-\mathrm{OH}\right)$ & 8.7 & 8.5 & 8.7 & 8.7 & 5.5 & 5.0 \\
& $\mathrm{p} K_{a, 2}\left(\mathrm{C}_{1}-\mathrm{OH}\right)$ & 12.5 & 12.6 & 12.5 & 14.1 & 9.2 & 8.0 \\
& $\mathrm{p} K_{a, 1}\left(\mathrm{C}_{4}-\mathrm{OH}\right)$ & 8.0 & 8.2 & 8.0 & 7.3 & 4.4 & 4.2 \\
& $\mathrm{p} K_{a, 2}\left(\mathrm{C}_{4}-\mathrm{OH}\right)$ & 13.2 & 12.8 & 13.2 & 15.5 & 10.3 & 8.9 \\
\hline \multirow{5}{*}{ CAM-B3LYP } & $\mathrm{p} K_{a, 1}\left(\mathrm{C}_{1}-\mathrm{OH}\right)$ & 7.7 & 7.5 & 5.4 & 7.5 & 4.5 & 4.2 \\
& $\mathrm{p} K_{a, 2}\left(\mathrm{C}_{1}-\mathrm{OH}\right)$ & 11.8 & 11.8 & 11.5 & 13.0 & 8.4 & 7.4 \\
& $\mathrm{p} K_{a, 1}\left(\mathrm{C}_{4}-\mathrm{OH}\right)$ & 7.0 & 7.1 & 3.9 & 6.1 & 3.4 & 3.3 \\
& $\mathrm{p} K_{a, 2}\left(\mathrm{C}_{4}-\mathrm{OH}\right)$ & 12.4 & 12.2 & 13.0 & 14.4 & 9.4 & 8.2 \\
\hline \multirow{5}{*}{ PBE0DH } & $\mathrm{p} K_{a, 1}\left(\mathrm{C}_{1}-\mathrm{OH}\right)$ & 9.6 & 9.5 & 7.6 & 9.6 & 6.5 & 6.1 \\
& $\mathrm{p} K_{a, 2}\left(\mathrm{C}_{1}-\mathrm{OH}\right)$ & 13.3 & 13.4 & 13.3 & 14.8 & 10.1 & 9.0 \\
& $\mathrm{p} K_{a, 1}\left(\mathrm{C}_{4}-\mathrm{OH}\right)$ & 8.9 & 8.9 & 5.9 & 8.0 & 5.4 & 5.2 \\
& $\mathrm{p} K_{a, 2}\left(\mathrm{C}_{4}-\mathrm{OH}\right)$ & 14.1 & 13.9 & 15.1 & 16.4 & 11.3 & 9.9 \\
\hline
\end{tabular}

Table S6. The theoretical estimated $\mathrm{p} K_{a, 1}$ and $\mathrm{p} K_{a, 2}$ of EP with SMD2 model.

\begin{tabular}{clcccccc}
\hline Functional & & UAHF & UAKS & UFF & UA0 & Bondi & Pauling \\
\hline \multirow{5}{*}{ PBE1PBE } & $\mathrm{p} K_{a, 1}\left(\mathrm{C}_{1}-\mathrm{OH}\right)$ & 3.3 & 2.9 & 3.3 & 5.3 & 0.6 & -0.7 \\
& $\mathrm{p} K_{a, 2}\left(\mathrm{C}_{1}-\mathrm{OH}\right)$ & 10.9 & 10.9 & 11.2 & 12.8 & 7.3 & 5.7 \\
& $\mathrm{p} K_{a, 1}\left(\mathrm{C}_{4}-\mathrm{OH}\right)$ & 4.2 & 4.2 & 2.7 & 4.8 & 0.9 & 0.0 \\
& $\mathrm{p} K_{a, 2}\left(\mathrm{C}_{4}-\mathrm{OH}\right)$ & 10.0 & 9.6 & 11.8 & 13.3 & 7.0 & 5.0 \\
\hline \multirow{5}{*}{ M062X } & $\mathrm{p} K_{a, 1}\left(\mathrm{C}_{1}-\mathrm{OH}\right)$ & 3.2 & 2.8 & 3.2 & 5.2 & 0.4 & -0.9 \\
& $\mathrm{p} K_{a, 2}\left(\mathrm{C}_{1}-\mathrm{OH}\right)$ & 10.7 & 10.7 & 10.7 & 12.6 & 6.9 & 5.4 \\
& $\mathrm{p} K_{a, 1}\left(\mathrm{C}_{4}-\mathrm{OH}\right)$ & 4.0 & 4.1 & 4.0 & 4.7 & 0.6 & -0.3 \\
& $\mathrm{p} K_{a, 2}\left(\mathrm{C}_{4}-\mathrm{OH}\right)$ & 9.8 & 9.3 & 9.8 & 13.1 & 6.7 & 4.7 \\
\hline & & & $\mathrm{S} 7$ & & & &
\end{tabular}




\begin{tabular}{cccccccc}
\hline & $\mathrm{p} K_{a, 1}\left(\mathrm{C}_{1}-\mathrm{OH}\right)$ & 2.2 & 1.8 & 2.0 & 3.9 & -0.6 & -1.7 \\
CAM-B3LYP & $\mathrm{p} K_{a, 2}\left(\mathrm{C}_{1}-\mathrm{OH}\right)$ & 9.9 & 9.9 & 9.8 & 11.4 & 6.0 & 4.7 \\
& $\mathrm{p} K_{a, 1}\left(\mathrm{C}_{4}-\mathrm{OH}\right)$ & 3.0 & 3.0 & 1.4 & 3.5 & -0.4 & -1.1 \\
& $\mathrm{p} K_{a, 2}\left(\mathrm{C}_{4}-\mathrm{OH}\right)$ & 9.0 & 8.7 & 10.4 & 11.9 & 5.8 & 4.0 \\
\hline \multirow{5}{*}{ PBE0DH } & $\mathrm{p} K_{a, 1}\left(\mathrm{C}_{1}-\mathrm{OH}\right)$ & 3.7 & 3.3 & 4.0 & 5.9 & 1.1 & -0.3 \\
& $\mathrm{p} K_{a, 2}\left(\mathrm{C}_{1}-\mathrm{OH}\right)$ & 11.1 & 11.2 & 11.6 & 13.2 & 7.6 & 6.0 \\
& $\mathrm{p} K_{a, 1}\left(\mathrm{C}_{4}-\mathrm{OH}\right)$ & 4.5 & 4.5 & 3.2 & 5.3 & 1.3 & 0.4 \\
& $\mathrm{p} K_{a, 2}\left(\mathrm{C}_{4}-\mathrm{OH}\right)$ & 10.3 & 10.0 & 12.3 & 13.7 & 7.4 & 5.4 \\
\hline
\end{tabular}




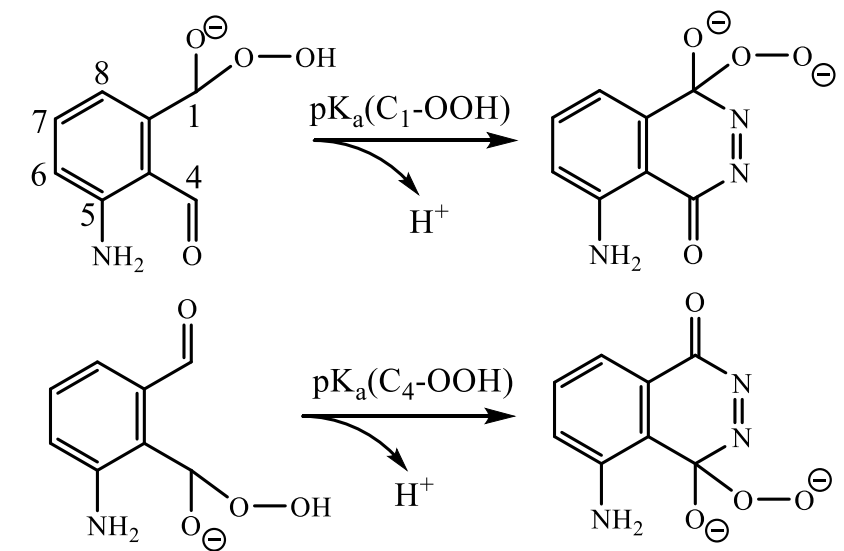

Scheme S4. The acidic dissociation of the endoperoxide (PI-).

Table S7. The theoretical estimated $\mathrm{p} K_{a}$ of PI- with SMD1 model.

\begin{tabular}{clcccccc}
\hline Method & & UAHF & UAKS & UFF & UA0 & Bondi & Pauling \\
\hline \multirow{2}{*}{ PBE1PBE } & $\mathrm{p} K_{a}\left(\mathrm{C}_{1}-\mathrm{OOH}\right)$ & 17.9 & 16.6 & 21.3 & 21.5 & 16.2 & 13.8 \\
& $\mathrm{p} K_{a}\left(\mathrm{C}_{4}-\mathrm{OOH}\right)$ & 18.9 & 17.3 & 22.7 & 22.9 & 17.6 & 15.5 \\
\multirow{2}{*}{ M062X } & $\mathrm{p} K_{a}\left(\mathrm{C}_{1}-\mathrm{OOH}\right)$ & 16.4 & 14.8 & 19.9 & 20.1 & 14.5 & 11.8 \\
& $\mathrm{p} K_{a}\left(\mathrm{C}_{4}-\mathrm{OOH}\right)$ & 14.8 & 13.0 & 19.8 & 19.6 & 13.6 & 10.9 \\
\multirow{2}{*}{ CAM-B3LYP } & $\mathrm{p} K_{a}\left(\mathrm{C}_{1}-\mathrm{OOH}\right)$ & 12.9 & 12.1 & 16.1 & 16.2 & 10.9 & 8.6 \\
& $\mathrm{p} K_{a}\left(\mathrm{C}_{4}-\mathrm{OOH}\right)$ & 12.5 & 10.9 & 17.3 & 17.1 & 11.4 & 8.9 \\
\multirow{2}{*}{ PBE0DH } & $\mathrm{p} K_{a}\left(\mathrm{C}_{1}-\mathrm{OOH}\right)$ & 17.2 & 16.0 & 20.7 & 20.7 & 15.4 & 12.8 \\
& $\mathrm{p} K_{a}\left(\mathrm{C}_{4}-\mathrm{OOH}\right)$ & 18.9 & 17.3 & 23.0 & 23.0 & 17.7 & 15.6 \\
\hline
\end{tabular}

Table S8. The theoretical estimated $\mathrm{p} K_{a}$ of PI $^{-}$with SMD2 model.

\begin{tabular}{clcccccc}
\hline Method & & UAHF & UAKS & UFF & UA0 & Bondi & Pauling \\
\hline \multirow{2}{*}{ PBE1PBE } & $\mathrm{p} K_{a}\left(\mathrm{C}_{1}-\mathrm{OOH}\right)$ & 7.7 & 5.8 & 14.3 & 14.0 & 6.4 & 1.7 \\
& $\mathrm{p} K_{a}\left(\mathrm{C}_{4}-\mathrm{OOH}\right)$ & 8.6 & 6.0 & 15.9 & 15.6 & 8.1 & 4.2 \\
\multirow{2}{*}{ M062X } & $\mathrm{p} K_{a}\left(\mathrm{C}_{1}-\mathrm{OOH}\right)$ & 7.3 & 5.3 & 13.3 & 13.1 & 5.5 & 0.9 \\
& $\mathrm{p} K_{a}\left(\mathrm{C}_{4}-\mathrm{OOH}\right)$ & 2.1 & -0.7 & 11.0 & 10.3 & 1.5 & -3.4 \\
\multirow{2}{*}{ CAM-B3LYP } & $\mathrm{p} K_{a}\left(\mathrm{C}_{1}-\mathrm{OOH}\right)$ & 2.9 & 1.6 & 8.8 & 8.7 & 1.3 & -2.7 \\
& $\mathrm{p} K_{a}\left(\mathrm{C}_{4}-\mathrm{OOH}\right)$ & -0.3 & -2.9 & 8.4 & 7.8 & -0.9 & -5.6 \\
\multirow{2}{*}{ PBE0DH } & $\mathrm{p} K_{a}\left(\mathrm{C}_{1}-\mathrm{OOH}\right)$ & 6.2 & 4.5 & 12.9 & 12.6 & 4.8 & 0.1 \\
& $\mathrm{p} K_{a}\left(\mathrm{C}_{4}-\mathrm{OOH}\right)$ & 7.1 & 4.3 & 15.1 & 14.5 & 6.5 & 2.2 \\
\hline
\end{tabular}



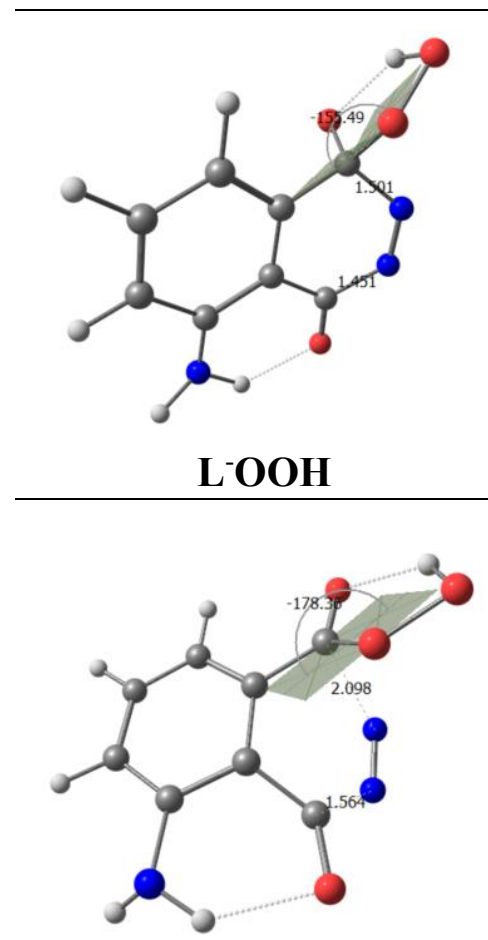

TS1-1

PI $^{2-}$

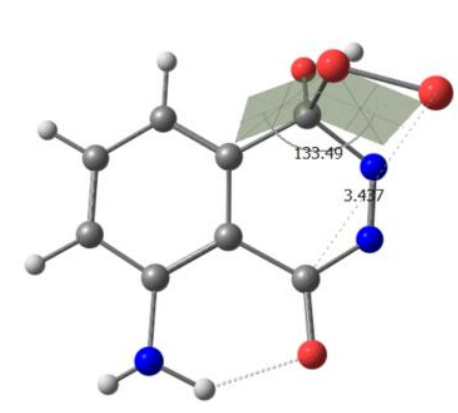

TS2-1

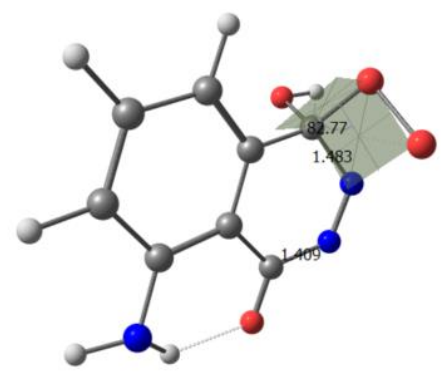

LOO $^{-}$

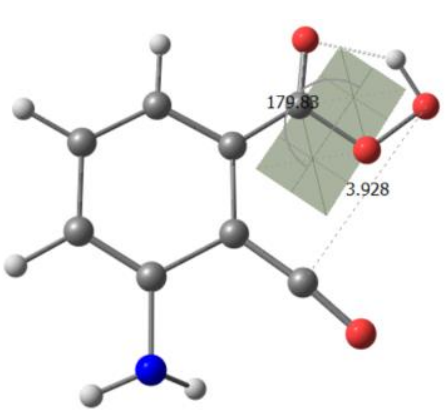

PI $^{-}$

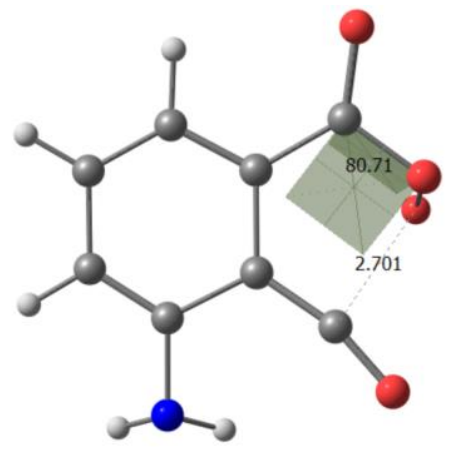

TS1-3

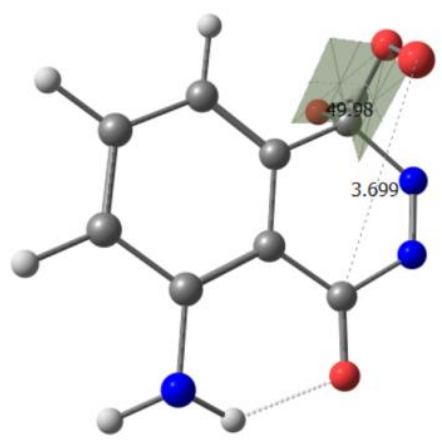

TS2-2

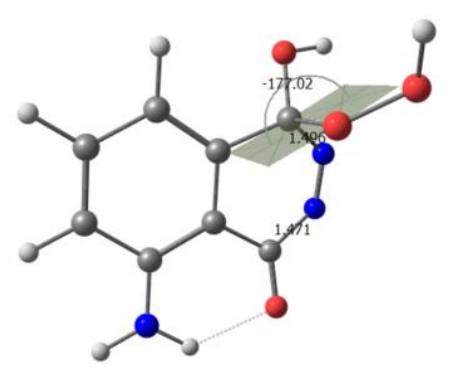

LHOOH

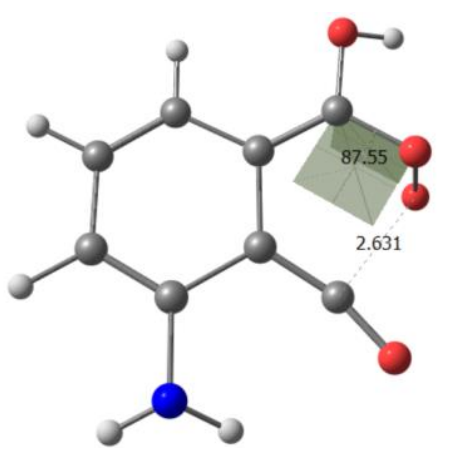

TS1-2

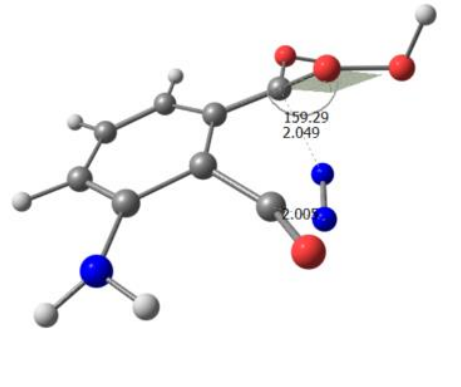

TS1-4

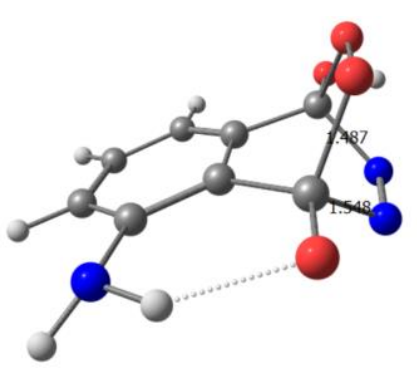

EP $^{-}$ 


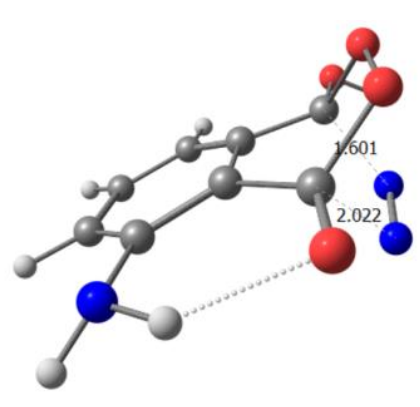

TS2-3

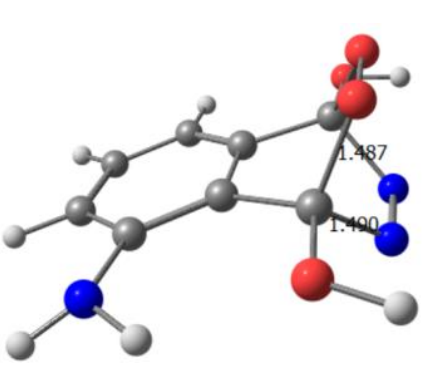

EP

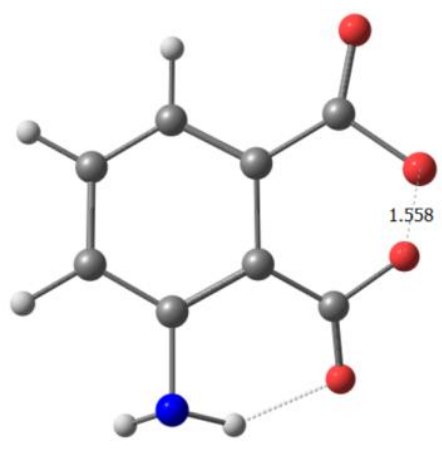

$\mathrm{TS}_{\mathrm{O}-\mathrm{O}}$

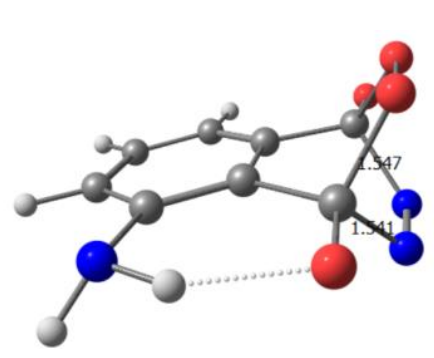

$\mathbf{E P}^{2-}$

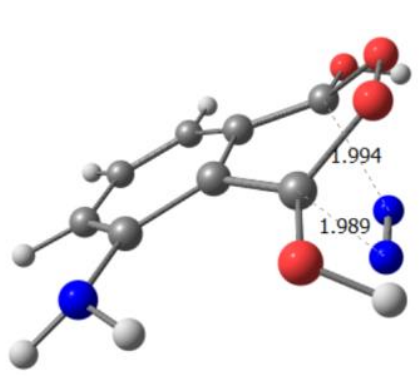

TS2-5

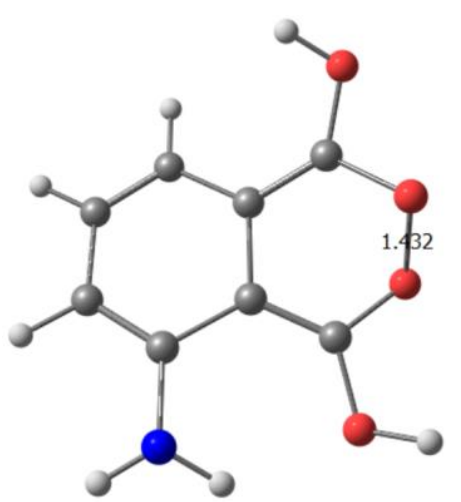

CP

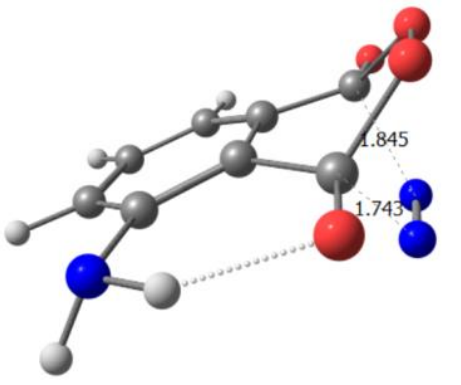

TS2-4

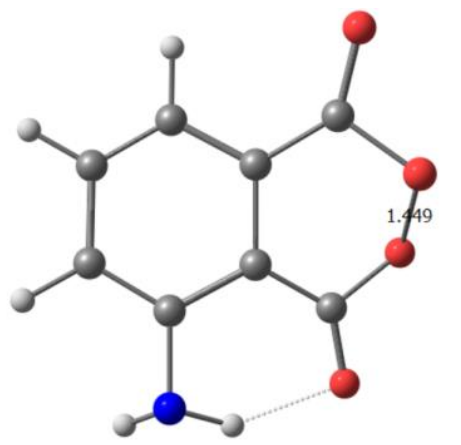

$\mathbf{C P}^{2-}$

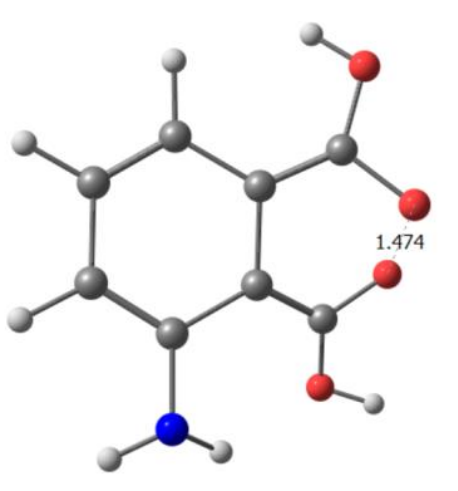

$\mathrm{TS}_{\mathrm{O}-\mathrm{O}}^{\prime}$

Figure S1. The geometric structure of the optimized stationary points for the 1-C isomers where the key geometric parameters including $\mathrm{C}-\mathrm{C}-\mathrm{O}-\mathrm{O}$ dihedral angels, $\mathrm{C}-\mathrm{O}$ bond lengths or $\mathrm{C}-\mathrm{N}$ bond lengths are labeled. 

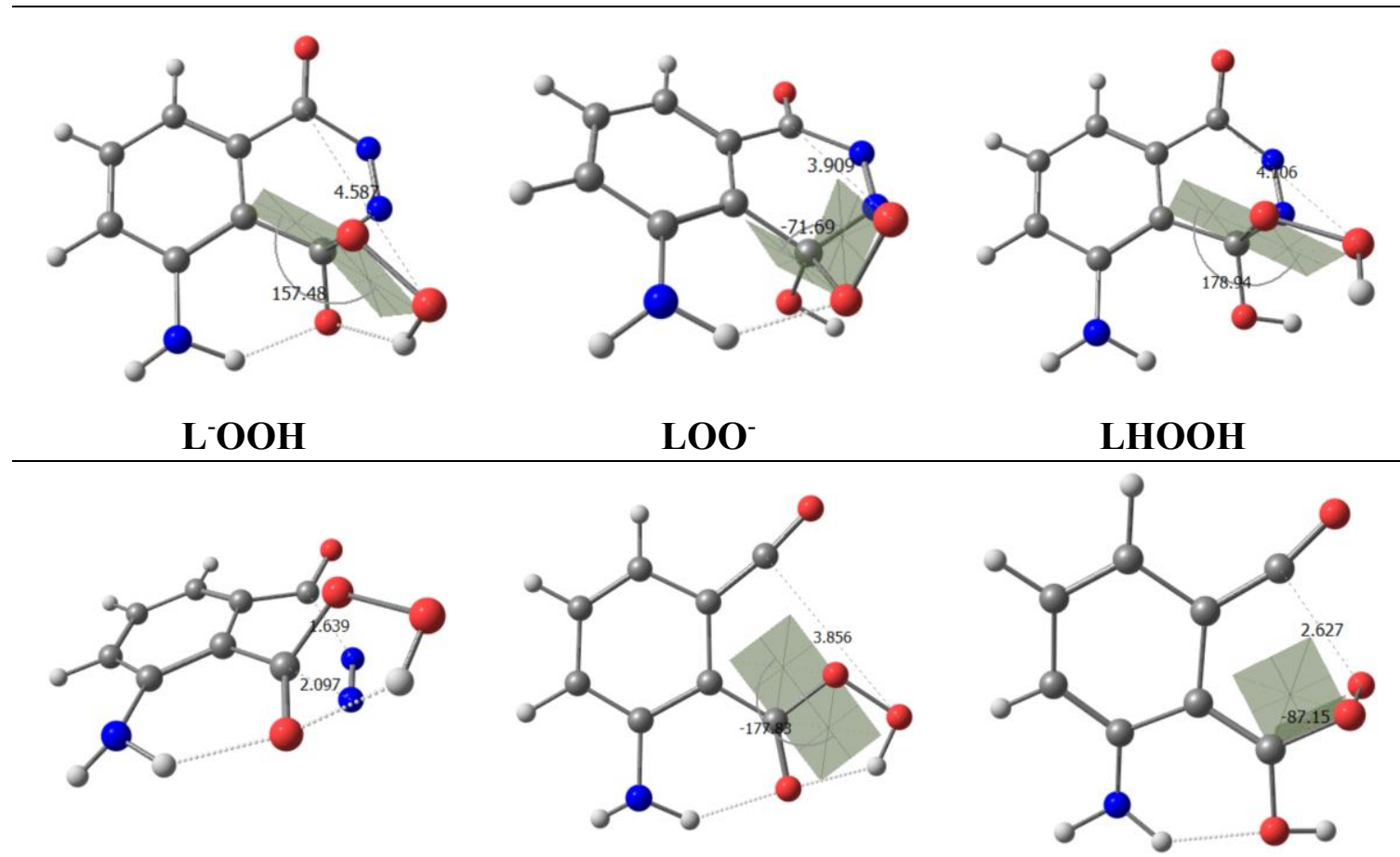

TS1-1

PI $^{-}$

TS1-2
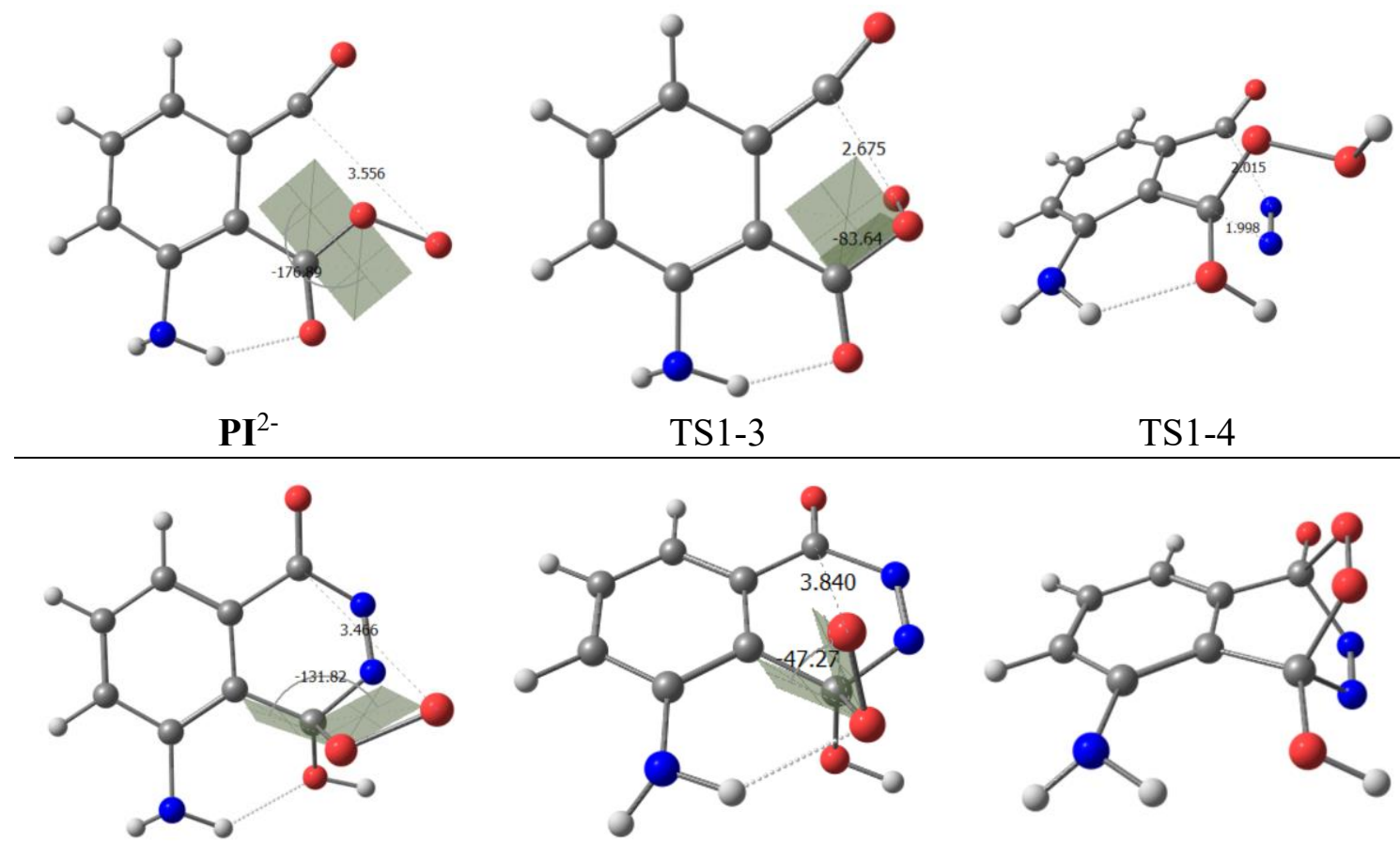

TS2-1
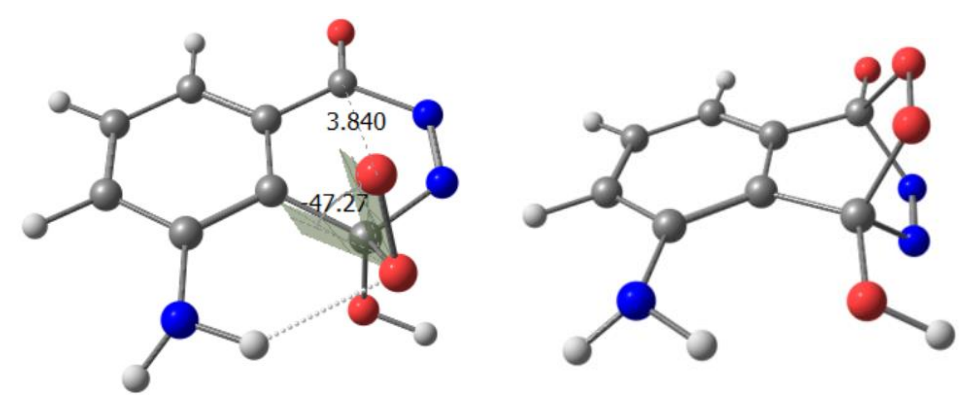

TS2-2

EP $^{-}$ 


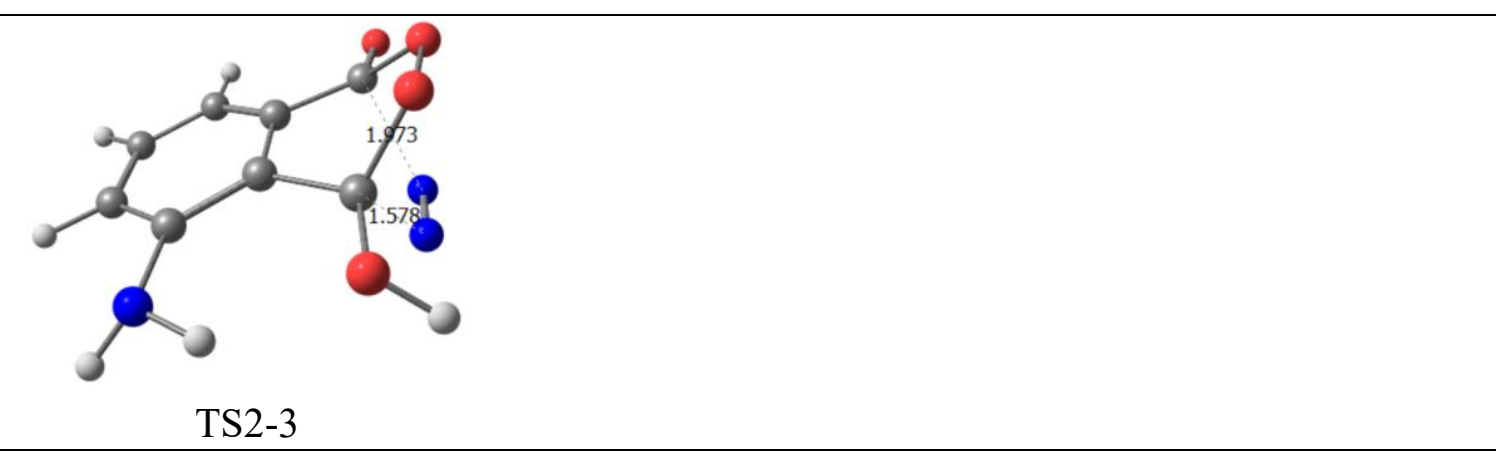

Figure S2. The geometric structure of the optimized stationary points for the 4-C isomers where the key geometric parameters including $\mathrm{C}-\mathrm{C}-\mathrm{O}-\mathrm{O}$ dihedral angels, $\mathrm{C}-\mathrm{O}$ bond lengths or $\mathrm{C}-\mathrm{N}$ bond lengths are labeled.
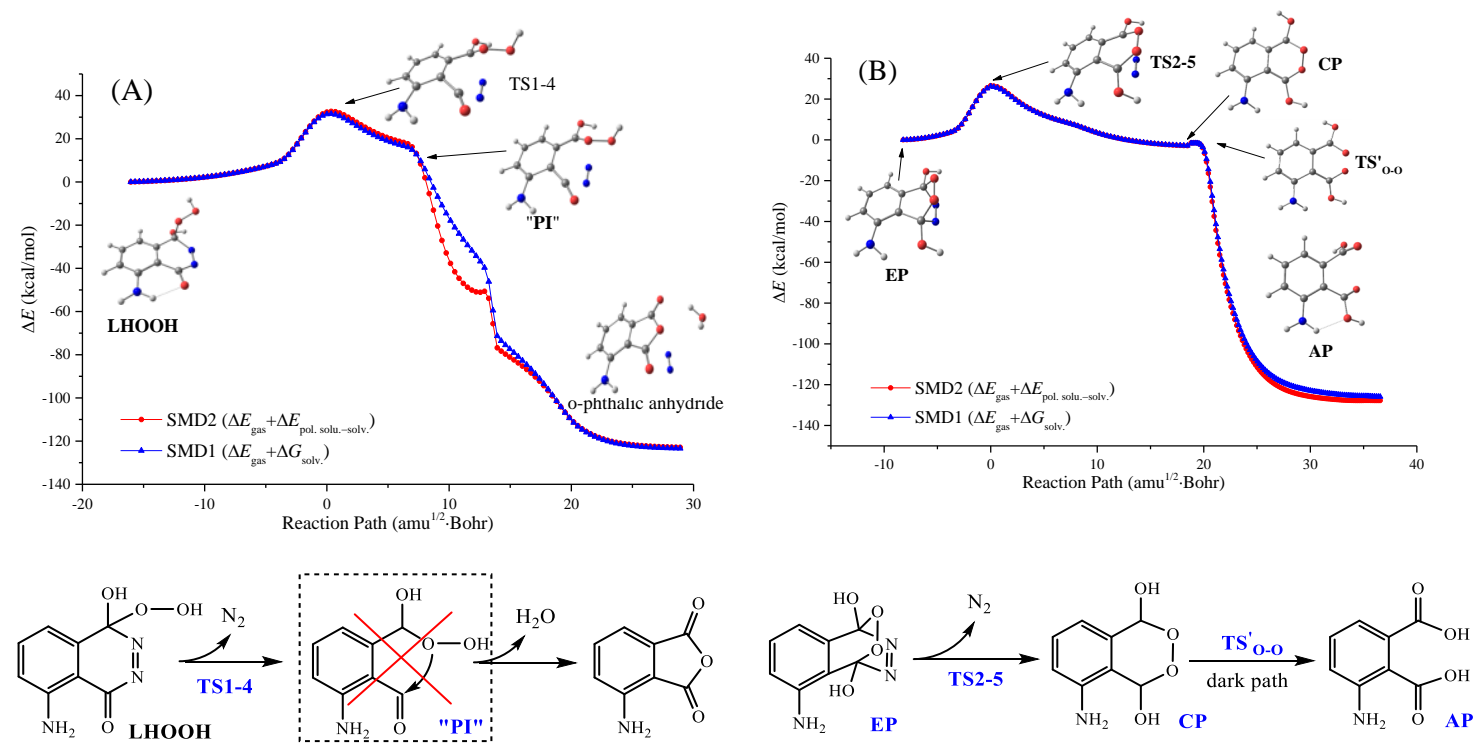

Figure S3. The $\mathrm{S}_{0}$ PECs with the SMD1 and SMD2 corrections at PBE1PBE/6-31+G*/Bondi level along the reaction path $\mathbf{L H O O H} \rightarrow \mathrm{TS} 1-4 \rightarrow$ "PI" $\rightarrow$ o-phthalic anhydride (A) and reaction path $\mathbf{E P} \rightarrow \mathbf{C P} \rightarrow \mathbf{A P}\left(\right.$ B). For energetic consistency, the $\mathrm{S}_{0}$ PECs for the $\mathbf{E P} \rightarrow \mathbf{C P} \rightarrow \mathbf{A P}$ path are corrected by adding the to free energy $(G)$ of $\mathrm{N}_{2}$. 


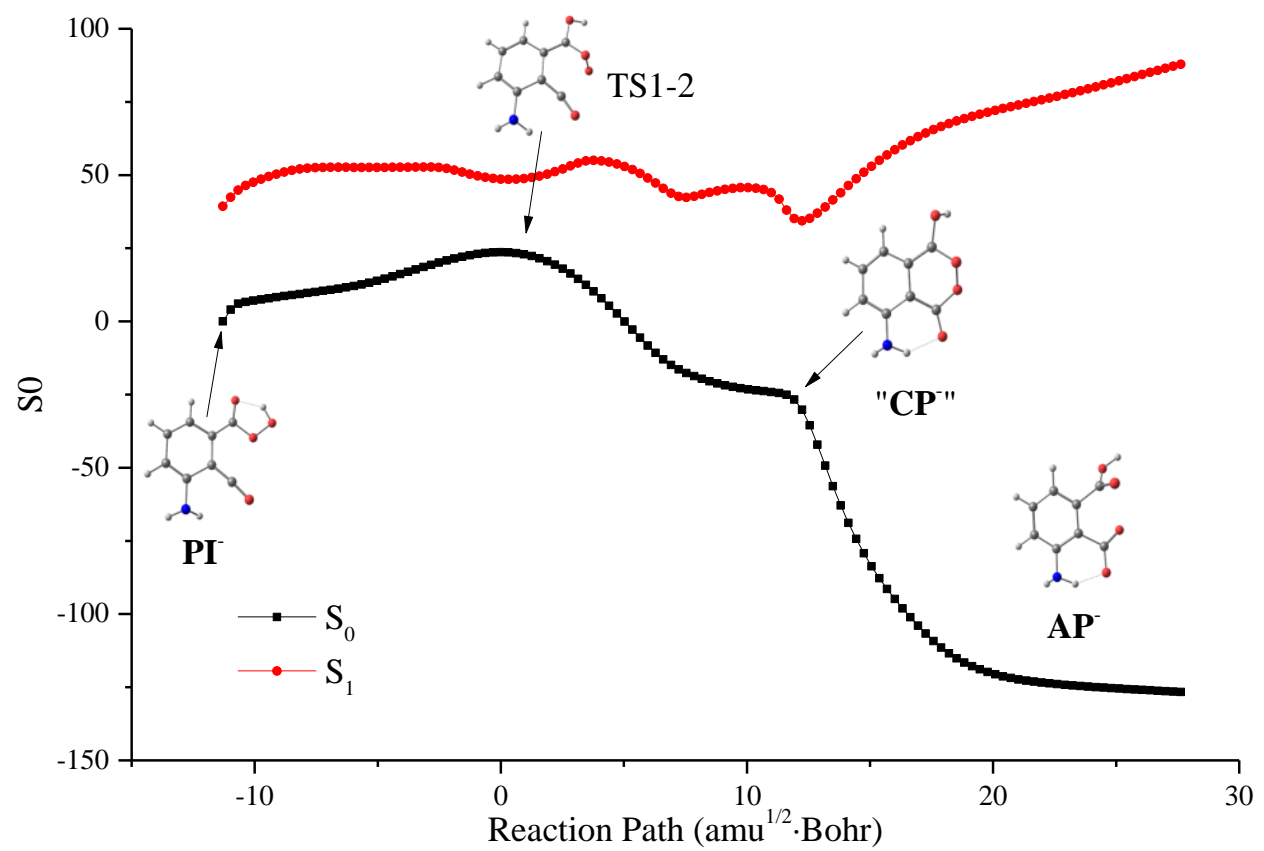

Figure S4. The $\mathrm{S}_{0}$ and $\mathrm{S}_{1}$ PECs along the $\mathrm{S}_{0}$ reaction path $\mathbf{P I} \rightarrow \mathrm{TS} 1-2 \rightarrow$ "CP$"$ " $\rightarrow \mathbf{A P}$ - at TDPBE1PBE/6-31+G*//PBE1PBE/6-31+G* level in gas phase.

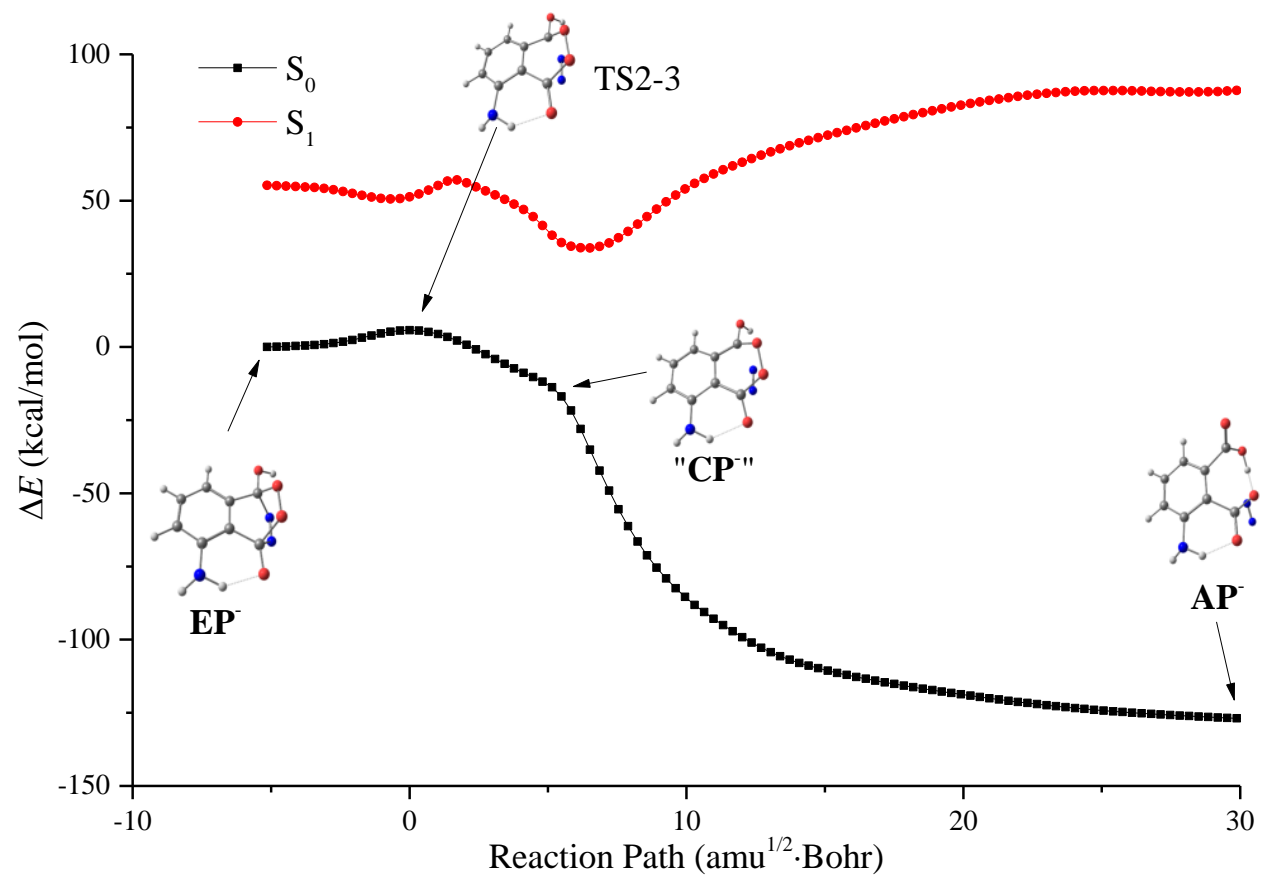

Figure S5. The $S_{0}$ and $S_{1}$ PECs along the $S_{0}$ reaction path $\mathbf{E P} \rightarrow$ TS2-3 $\rightarrow$ "CP'" $\rightarrow$ AP' at TDPBE1PBE/6-31+G*//PBE1PBE/6-31+G* level in gas phase. 


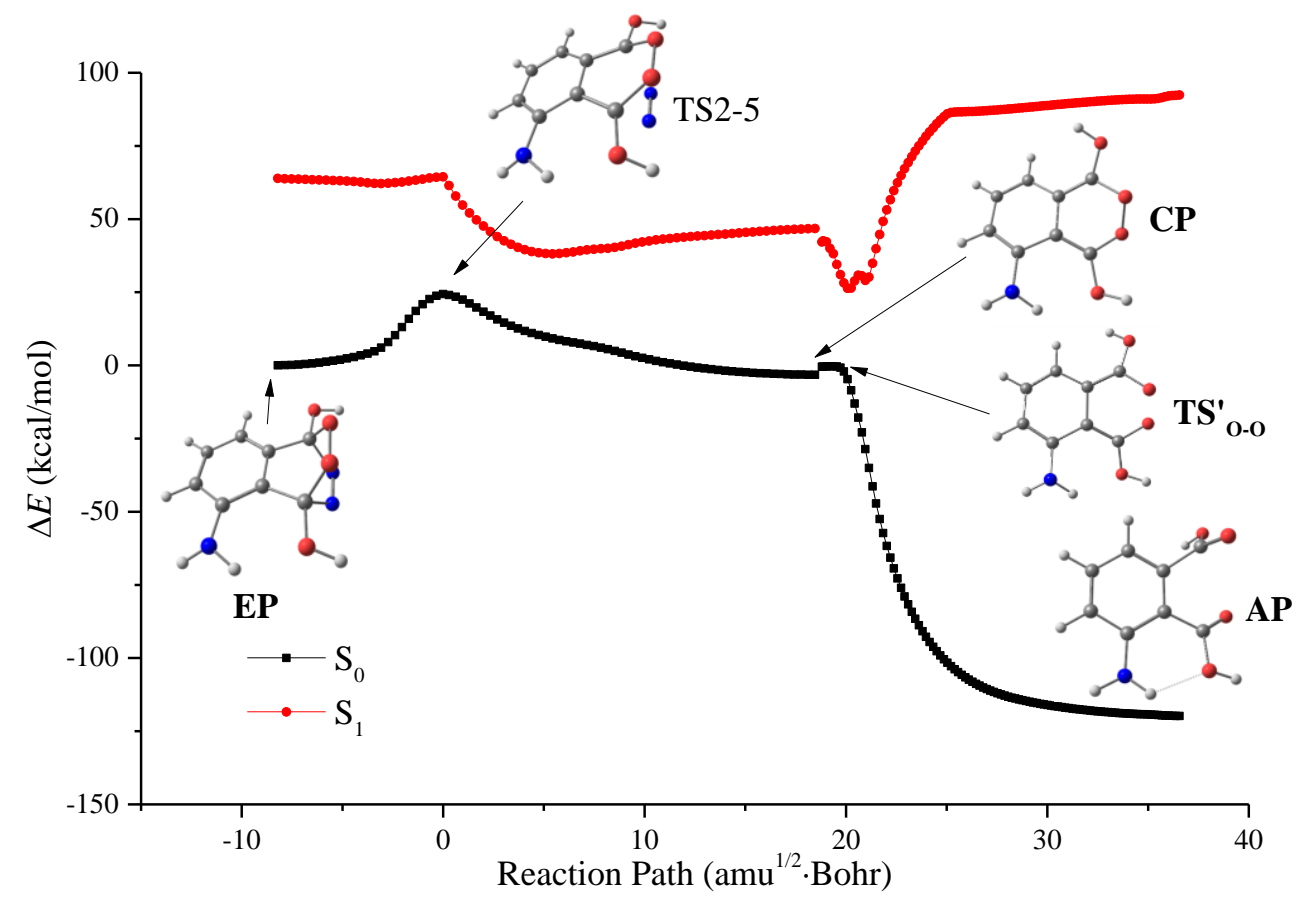

Figure S6. The $S_{0}$ and $S_{1}$ PECs along the $S_{0}$ reaction path $\mathbf{E P} \rightarrow T S 2-5 \rightarrow \mathbf{C P} \rightarrow \mathbf{A P}$ at TD-PBE1PBE/6$31+\mathrm{G}^{*} / / \mathrm{PBE} 1 \mathrm{PBE} / 6-31+\mathrm{G}^{*}$ level in gas phase. 
The optimized Cartesian coordinates $(\AA)$ in gas phase used in $p K_{a}$ estimation. at PBE1PBE/6-31+G*, M062X/6-31+G*, CAM-B3LYP/6-31+G* and PBE0DH/631+G* levels, respectively.

\begin{tabular}{|c|c|c|c|c|c|c|c|}
\hline \multicolumn{4}{|c|}{ Luminol, $\mathbf{L H}_{2}$ (PBE1PBE/6-31+G*) } & \multicolumn{4}{|c|}{ Luminol Dianion, $\mathbf{L}^{2-}\left(\mathrm{PBE} 1 \mathrm{PBE} / 6-31+\mathrm{G}^{*}\right)$} \\
\hline $\mathrm{C}$ & 13.577 & 14.901 & 13.186 & $\mathrm{C}$ & 13.620 & 14.933 & 13.195 \\
\hline $\mathrm{C}$ & 14.290 & 13.970 & 12.380 & $\mathrm{C}$ & 14.431 & 14.006 & 12.468 \\
\hline $\mathrm{C}$ & 14.821 & 14.389 & 11.141 & $\mathrm{C}$ & 14.990 & 14.408 & 11.231 \\
\hline $\mathrm{C}$ & 14.677 & 15.693 & 10.690 & $\mathrm{C}$ & 14.728 & 15.696 & 10.717 \\
\hline $\mathrm{C}$ & 13.976 & 16.601 & 11.486 & $\mathrm{C}$ & 13.916 & 16.571 & 11.415 \\
\hline $\mathrm{C}$ & 13.439 & 16.218 & 12.703 & $\mathrm{C}$ & 13.365 & 16.195 & 12.657 \\
\hline $\mathrm{H}$ & 15.111 & 15.973 & 9.737 & $\mathrm{H}$ & 15.182 & 15.949 & 9.762 \\
\hline $\mathrm{H}$ & 13.846 & 17.626 & 11.149 & $\mathrm{H}$ & 13.703 & 17.566 & 11.015 \\
\hline $\mathrm{H}$ & 12.900 & 16.942 & 13.311 & $\mathrm{H}$ & 12.755 & 16.899 & 13.228 \\
\hline $\mathrm{C}$ & 14.425 & 12.576 & 12.799 & $\mathrm{C}$ & 14.662 & 12.631 & 12.915 \\
\hline $\mathrm{C}$ & 15.591 & 13.447 & 10.297 & $\mathrm{C}$ & 15.827 & 13.466 & 10.477 \\
\hline $\mathrm{O}$ & 13.892 & 12.076 & 13.797 & $\mathrm{O}$ & 14.116 & 12.190 & 14.015 \\
\hline $\mathrm{N}$ & 15.212 & 11.772 & 12.011 & $\mathrm{~N}$ & 15.425 & 11.836 & 12.163 \\
\hline $\mathrm{N}$ & 15.610 & 12.143 & 10.728 & $\mathrm{~N}$ & 16.002 & 12.234 & 10.992 \\
\hline $\mathrm{N}$ & 13.071 & 14.553 & 14.396 & $\mathrm{~N}$ & 13.137 & 14.567 & 14.455 \\
\hline $\mathrm{H}$ & 12.440 & 15.181 & 14.867 & $\mathrm{H}$ & 12.176 & 14.852 & 14.607 \\
\hline $\mathrm{H}$ & 13.071 & 13.568 & 14.641 & $\mathrm{H}$ & 13.314 & 13.535 & 14.559 \\
\hline $\mathrm{O}$ & 16.182 & 13.761 & 9.274 & $\mathrm{O}$ & 16.360 & 13.827 & 9.370 \\
\hline $\mathrm{H}$ & 14.988 & 10.787 & 12.093 & & & & \\
\hline $\mathrm{H}$ & 16.393 & 11.603 & 10.377 & & & & \\
\hline
\end{tabular}




\begin{tabular}{|c|c|c|c|c|c|c|c|}
\hline \multicolumn{8}{|c|}{ Luminol monoanion, LH' (PBE1PBE/6-31+G*) } \\
\hline \multicolumn{4}{|c|}{$1-\mathrm{C}$} & \multicolumn{4}{|c|}{$4-\mathrm{C}$} \\
\hline $\mathrm{C}$ & 13.576 & 14.921 & 13.190 & $\mathrm{C}$ & 13.620 & 14.909 & 13.184 \\
\hline $\mathrm{C}$ & 14.335 & 13.990 & 12.427 & $\mathrm{C}$ & 14.362 & 13.975 & 12.399 \\
\hline $\mathrm{C}$ & 14.922 & 14.382 & 11.208 & $\mathrm{C}$ & 14.888 & 14.412 & 11.168 \\
\hline $\mathrm{C}$ & 14.742 & 15.686 & 10.732 & $\mathrm{C}$ & 14.684 & 15.713 & 10.689 \\
\hline $\mathrm{C}$ & 13.979 & 16.583 & 11.464 & $\mathrm{C}$ & 13.933 & 16.595 & 11.450 \\
\hline $\mathrm{C}$ & 13.403 & 16.212 & 12.681 & $\mathrm{C}$ & 13.410 & 16.201 & 12.682 \\
\hline $\mathrm{H}$ & 15.212 & 15.944 & 9.787 & $\mathrm{H}$ & 15.118 & 15.985 & 9.732 \\
\hline $\mathrm{H}$ & 13.827 & 17.597 & 11.095 & $\mathrm{H}$ & 13.752 & 17.609 & 11.094 \\
\hline $\mathrm{H}$ & 12.826 & 16.934 & 13.259 & $\mathrm{H}$ & 12.840 & 16.908 & 13.285 \\
\hline $\mathrm{C}$ & 14.491 & 12.603 & 12.853 & $\mathrm{C}$ & 14.540 & 12.560 & 12.831 \\
\hline $\mathrm{C}$ & 15.735 & 13.425 & 10.393 & $\mathrm{C}$ & 15.670 & 13.473 & 10.355 \\
\hline $\mathrm{O}$ & 13.980 & 12.089 & 13.889 & $\mathrm{O}$ & 14.002 & 12.143 & 13.907 \\
\hline $\mathrm{N}$ & 15.238 & 11.861 & 12.016 & $\mathrm{~N}$ & 15.253 & 11.732 & 12.069 \\
\hline $\mathrm{N}$ & 15.872 & 12.167 & 10.836 & $\mathrm{~N}$ & 15.774 & 12.248 & 10.911 \\
\hline $\mathrm{N}$ & 13.071 & 14.564 & 14.427 & $\mathrm{~N}$ & 13.165 & 14.552 & 14.433 \\
\hline $\mathrm{H}$ & 12.217 & 15.029 & 14.700 & $\mathrm{H}$ & 12.328 & 15.020 & 14.751 \\
\hline $\mathrm{H}$ & 13.105 & 13.553 & 14.584 & $\mathrm{H}$ & 13.236 & 13.529 & 14.572 \\
\hline $\mathrm{O}$ & 16.262 & 13.822 & 9.326 & $\mathrm{O}$ & 16.203 & 13.749 & 9.260 \\
\hline $\mathrm{H}$ & 15.351 & 10.894 & 12.290 & $\mathrm{H}$ & 16.306 & 11.574 & 10.377 \\
\hline
\end{tabular}




\begin{tabular}{|c|c|c|c|c|c|c|c|}
\hline \multicolumn{4}{|c|}{ Luminol, $\mathbf{L H}_{2}\left(\mathrm{M} 062 \mathrm{X} / 6-31+\mathrm{G}^{*}\right)$} & \multicolumn{4}{|c|}{ Luminol Dianion, $\mathbf{L}^{2-}\left(\mathrm{M} 062 \mathrm{X} / 6-31+\mathrm{G}^{*}\right)$} \\
\hline $\mathrm{C}$ & 13.580 & 14.896 & 13.187 & $\mathrm{C}$ & 13.623 & 14.931 & 13.194 \\
\hline $\mathrm{C}$ & 14.289 & 13.970 & 12.380 & $\mathrm{C}$ & 14.434 & 14.005 & 12.467 \\
\hline $\mathrm{C}$ & 14.816 & 14.389 & 11.141 & $\mathrm{C}$ & 14.986 & 14.406 & 11.234 \\
\hline $\mathrm{C}$ & 14.673 & 15.691 & 10.688 & $\mathrm{C}$ & 14.727 & 15.695 & 10.716 \\
\hline $\mathrm{C}$ & 13.973 & 16.599 & 11.486 & $\mathrm{C}$ & 13.919 & 16.570 & 11.416 \\
\hline $\mathrm{C}$ & 13.441 & 16.216 & 12.704 & $\mathrm{C}$ & 13.371 & 16.193 & 12.659 \\
\hline $\mathrm{H}$ & 15.105 & 15.969 & 9.734 & $\mathrm{H}$ & 15.180 & 15.944 & 9.761 \\
\hline $\mathrm{H}$ & 13.843 & 17.624 & 11.151 & $\mathrm{H}$ & 13.707 & 17.564 & 11.018 \\
\hline $\mathrm{H}$ & 12.904 & 16.937 & 13.315 & $\mathrm{H}$ & 12.763 & 16.895 & 13.232 \\
\hline $\mathrm{C}$ & 14.427 & 12.567 & 12.797 & $\mathrm{C}$ & 14.671 & 12.621 & 12.915 \\
\hline $\mathrm{C}$ & 15.592 & 13.444 & 10.293 & $\mathrm{C}$ & 15.828 & 13.462 & 10.473 \\
\hline $\mathrm{O}$ & 13.892 & 12.070 & 13.787 & $\mathrm{O}$ & 14.125 & 12.189 & 14.018 \\
\hline $\mathrm{N}$ & 15.222 & 11.764 & 12.010 & $\mathrm{~N}$ & 15.429 & 11.833 & 12.163 \\
\hline $\mathrm{N}$ & 15.611 & 12.134 & 10.715 & $\mathrm{~N}$ & 16.006 & 12.237 & 10.982 \\
\hline $\mathrm{N}$ & 13.073 & 14.561 & 14.404 & $\mathrm{~N}$ & 13.128 & 14.575 & 14.461 \\
\hline $\mathrm{H}$ & 12.449 & 15.204 & 14.866 & $\mathrm{H}$ & 12.161 & 14.853 & 14.590 \\
\hline $\mathrm{H}$ & 13.050 & 13.582 & 14.667 & $\mathrm{H}$ & 13.301 & 13.548 & 14.576 \\
\hline $\mathrm{O}$ & 16.181 & 13.768 & 9.277 & $\mathrm{O}$ & 16.350 & 13.841 & 9.365 \\
\hline $\mathrm{H}$ & 14.977 & 10.781 & 12.082 & & & & \\
\hline $\mathrm{H}$ & 16.415 & 11.614 & 10.380 & & & & \\
\hline
\end{tabular}




\begin{tabular}{|c|c|c|c|c|c|c|c|}
\hline \multicolumn{8}{|c|}{ Luminol monoanion, $\mathbf{L H}^{-}\left(\mathrm{M} 062 \mathrm{X} / 6-31+\mathrm{G}^{*}\right)$} \\
\hline \multicolumn{4}{|c|}{$1-\mathrm{C}$} & \multicolumn{4}{|c|}{$4-\mathrm{C}$} \\
\hline $\mathrm{C}$ & 13.577 & 14.917 & 13.189 & $\mathrm{C}$ & 13.622 & 14.904 & 13.185 \\
\hline $\mathrm{C}$ & 14.336 & 13.989 & 12.427 & $\mathrm{C}$ & 14.363 & 13.972 & 12.399 \\
\hline $\mathrm{C}$ & 14.918 & 14.380 & 11.209 & $\mathrm{C}$ & 14.883 & 14.411 & 11.170 \\
\hline $\mathrm{C}$ & 14.741 & 15.684 & 10.731 & $\mathrm{C}$ & 14.681 & 15.710 & 10.688 \\
\hline $\mathrm{C}$ & 13.981 & 16.582 & 11.466 & $\mathrm{C}$ & 13.934 & 16.593 & 11.450 \\
\hline $\mathrm{C}$ & 13.407 & 16.209 & 12.684 & $\mathrm{C}$ & 13.415 & 16.197 & 12.684 \\
\hline $\mathrm{H}$ & 15.209 & 15.941 & 9.787 & $\mathrm{H}$ & 15.113 & 15.980 & 9.730 \\
\hline $\mathrm{H}$ & 13.831 & 17.596 & 11.100 & $\mathrm{H}$ & 13.753 & 17.607 & 11.097 \\
\hline $\mathrm{H}$ & 12.833 & 16.929 & 13.265 & $\mathrm{H}$ & 12.849 & 16.903 & 13.290 \\
\hline $\mathrm{C}$ & 14.499 & 12.593 & 12.852 & $\mathrm{C}$ & 14.547 & 12.547 & 12.829 \\
\hline $\mathrm{C}$ & 15.736 & 13.420 & 10.386 & $\mathrm{C}$ & 15.671 & 13.471 & 10.350 \\
\hline $\mathrm{O}$ & 13.991 & 12.084 & 13.886 & $\mathrm{O}$ & 14.008 & 12.137 & 13.905 \\
\hline $\mathrm{N}$ & 15.245 & 11.857 & 12.015 & $\mathrm{~N}$ & 15.258 & 11.725 & 12.067 \\
\hline $\mathrm{N}$ & 15.880 & 12.167 & 10.825 & $\mathrm{~N}$ & 15.782 & 12.248 & 10.900 \\
\hline $\mathrm{N}$ & 13.063 & 14.572 & 14.433 & $\mathrm{~N}$ & 13.160 & 14.562 & 14.441 \\
\hline $\mathrm{H}$ & 12.211 & 15.050 & 14.690 & $\mathrm{H}$ & 12.321 & 15.038 & 14.741 \\
\hline $\mathrm{H}$ & 13.073 & 13.565 & 14.603 & $\mathrm{H}$ & 13.211 & 13.545 & 14.597 \\
\hline $\mathrm{O}$ & 16.252 & 13.832 & 9.321 & $\mathrm{O}$ & 16.195 & 13.761 & 9.257 \\
\hline $\mathrm{H}$ & 15.363 & 10.889 & 12.286 & $\mathrm{H}$ & 16.315 & 11.575 & 10.366 \\
\hline
\end{tabular}




\begin{tabular}{|c|c|c|c|c|c|c|c|}
\hline \multicolumn{4}{|c|}{ Luminol, $\mathbf{L H}_{2}$ (CAM-B3LYP/6-31+G*) } & \multicolumn{4}{|c|}{ Luminol Dianion, $\mathbf{L}^{2-}\left(\mathrm{CAM}-\mathrm{B} 3 \mathrm{LYP} / 6-31+\mathrm{G}^{*}\right)$} \\
\hline $\mathrm{C}$ & 13.575 & 14.898 & 13.181 & $\mathrm{C}$ & 13.622 & 14.932 & 13.192 \\
\hline $\mathrm{C}$ & 14.285 & 13.970 & 12.377 & $\mathrm{C}$ & 14.431 & 14.004 & 12.464 \\
\hline $\mathrm{C}$ & 14.819 & 14.390 & 11.141 & $\mathrm{C}$ & 14.984 & 14.405 & 11.233 \\
\hline $\mathrm{C}$ & 14.677 & 15.691 & 10.693 & $\mathrm{C}$ & 14.726 & 15.693 & 10.718 \\
\hline $\mathrm{C}$ & 13.977 & 16.599 & 11.489 & $\mathrm{C}$ & 13.920 & 16.567 & 11.417 \\
\hline $\mathrm{C}$ & 13.440 & 16.216 & 12.702 & $\mathrm{C}$ & 13.373 & 16.192 & 12.658 \\
\hline $\mathrm{H}$ & 15.111 & 15.973 & 9.741 & $\mathrm{H}$ & 15.178 & 15.943 & 9.764 \\
\hline $\mathrm{H}$ & 13.851 & 17.625 & 11.153 & $\mathrm{H}$ & 13.710 & 17.562 & 11.019 \\
\hline $\mathrm{H}$ & 12.902 & 16.938 & 13.311 & $\mathrm{H}$ & 12.767 & 16.896 & 13.231 \\
\hline $\mathrm{C}$ & 14.427 & 12.573 & 12.798 & $\mathrm{C}$ & 14.671 & 12.624 & 12.913 \\
\hline $\mathrm{C}$ & 15.589 & 13.445 & 10.295 & $\mathrm{C}$ & 15.826 & 13.462 & 10.476 \\
\hline $\mathrm{O}$ & 13.897 & 12.077 & 13.795 & $\mathrm{O}$ & 14.123 & 12.193 & 14.018 \\
\hline $\mathrm{N}$ & 15.215 & 11.768 & 12.012 & $\mathrm{~N}$ & 15.430 & 11.835 & 12.167 \\
\hline $\mathrm{N}$ & 15.611 & 12.139 & 10.719 & $\mathrm{~N}$ & 16.008 & 12.240 & 10.982 \\
\hline $\mathrm{N}$ & 13.057 & 14.553 & 14.390 & $\mathrm{~N}$ & 13.126 & 14.572 & 14.455 \\
\hline $\mathrm{H}$ & 12.450 & 15.197 & 14.873 & $\mathrm{H}$ & 12.163 & 14.853 & 14.596 \\
\hline $\mathrm{H}$ & 13.062 & 13.575 & 14.655 & $\mathrm{H}$ & 13.305 & 13.546 & 14.575 \\
\hline $\mathrm{O}$ & 16.179 & 13.765 & 9.274 & $\mathrm{O}$ & 16.349 & 13.841 & 9.366 \\
\hline $\mathrm{H}$ & 14.992 & 10.783 & 12.094 & & & & \\
\hline $\mathrm{H}$ & 16.398 & 11.604 & 10.370 & & & & \\
\hline
\end{tabular}




\begin{tabular}{|c|c|c|c|c|c|c|c|}
\hline \multicolumn{8}{|c|}{ Luminol monoanion, $\mathbf{L H}^{-}\left(\mathrm{CAM}-\mathrm{B} 3 \mathrm{LYP} / 6-31+\mathrm{G}^{*}\right)$} \\
\hline \multicolumn{4}{|c|}{$1-\mathrm{C}$} & \multicolumn{4}{|c|}{$4-\mathrm{C}$} \\
\hline $\mathrm{C}$ & 13.577 & 14.919 & 13.186 & $\mathrm{C}$ & 13.621 & 14.907 & 13.182 \\
\hline $\mathrm{C}$ & 14.334 & 13.990 & 12.424 & $\mathrm{C}$ & 14.361 & 13.975 & 12.397 \\
\hline $\mathrm{C}$ & 14.917 & 14.381 & 11.208 & $\mathrm{C}$ & 14.883 & 14.411 & 11.170 \\
\hline $\mathrm{C}$ & 14.741 & 15.684 & 10.733 & $\mathrm{C}$ & 14.680 & 15.710 & 10.690 \\
\hline $\mathrm{C}$ & 13.982 & 16.581 & 11.466 & $\mathrm{C}$ & 13.935 & 16.593 & 11.451 \\
\hline $\mathrm{C}$ & 13.407 & 16.210 & 12.683 & $\mathrm{C}$ & 13.416 & 16.198 & 12.683 \\
\hline $\mathrm{H}$ & 15.209 & 15.942 & 9.790 & $\mathrm{H}$ & 15.112 & 15.982 & 9.734 \\
\hline $\mathrm{H}$ & 13.834 & 17.595 & 11.100 & $\mathrm{H}$ & 13.757 & 17.606 & 11.097 \\
\hline $\mathrm{H}$ & 12.834 & 16.930 & 13.263 & $\mathrm{H}$ & 12.851 & 16.905 & 13.289 \\
\hline $\mathrm{C}$ & 14.496 & 12.598 & 12.849 & $\mathrm{C}$ & 14.545 & 12.553 & 12.828 \\
\hline $\mathrm{C}$ & 15.733 & 13.421 & 10.390 & $\mathrm{C}$ & 15.669 & 13.471 & 10.353 \\
\hline $\mathrm{O}$ & 13.986 & 12.091 & 13.887 & $\mathrm{O}$ & 14.006 & 12.143 & 13.906 \\
\hline $\mathrm{N}$ & 15.242 & 11.857 & 12.019 & $\mathrm{~N}$ & 15.255 & 11.728 & 12.071 \\
\hline $\mathrm{N}$ & 15.878 & 12.170 & 10.827 & $\mathrm{~N}$ & 15.781 & 12.248 & 10.901 \\
\hline $\mathrm{N}$ & 13.060 & 14.566 & 14.424 & $\mathrm{~N}$ & 13.157 & 14.556 & 14.434 \\
\hline $\mathrm{H}$ & 12.212 & 15.043 & 14.698 & $\mathrm{H}$ & 12.322 & 15.031 & 14.747 \\
\hline $\mathrm{H}$ & 13.089 & 13.559 & 14.596 & $\mathrm{H}$ & 13.224 & 13.539 & 14.587 \\
\hline $\mathrm{O}$ & 16.253 & 13.830 & 9.322 & $\mathrm{O}$ & 16.194 & 13.757 & 9.258 \\
\hline $\mathrm{H}$ & 15.359 & 10.890 & 12.290 & $\mathrm{H}$ & 16.313 & 11.574 & 10.368 \\
\hline
\end{tabular}




\begin{tabular}{|c|c|c|c|c|c|c|c|}
\hline \multicolumn{4}{|c|}{ 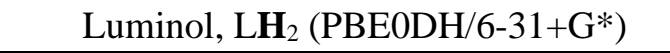 } & \multicolumn{4}{|c|}{ Luminol Dianion, $\mathbf{L}^{2-}\left(\mathrm{PBE} 0 \mathrm{DH} / 6-31+\mathrm{G}^{*}\right)$} \\
\hline $\mathrm{C}$ & 13.589 & 14.899 & 13.188 & $\mathrm{C}$ & 13.624 & 14.931 & 13.193 \\
\hline $\mathrm{C}$ & 14.298 & 13.973 & 12.384 & $\mathrm{C}$ & 14.433 & 14.007 & 12.469 \\
\hline $\mathrm{C}$ & 14.816 & 14.387 & 11.143 & $\mathrm{C}$ & 14.986 & 14.406 & 11.235 \\
\hline $\mathrm{C}$ & 14.670 & 15.686 & 10.690 & $\mathrm{C}$ & 14.727 & 15.691 & 10.721 \\
\hline $\mathrm{C}$ & 13.972 & 16.591 & 11.485 & $\mathrm{C}$ & 13.919 & 16.564 & 11.417 \\
\hline $\mathrm{C}$ & 13.446 & 16.211 & 12.704 & $\mathrm{C}$ & 13.373 & 16.190 & 12.657 \\
\hline $\mathrm{H}$ & 15.098 & 15.963 & 9.737 & $\mathrm{H}$ & 15.178 & 15.940 & 9.767 \\
\hline $\mathrm{H}$ & 13.839 & 17.612 & 11.148 & $\mathrm{H}$ & 13.708 & 17.555 & 11.020 \\
\hline $\mathrm{H}$ & 12.913 & 16.934 & 13.312 & $\mathrm{H}$ & 12.767 & 16.892 & 13.228 \\
\hline $\mathrm{C}$ & 14.423 & 12.577 & 12.794 & $\mathrm{C}$ & 14.663 & 12.632 & 12.913 \\
\hline $\mathrm{C}$ & 15.590 & 13.448 & 10.305 & $\mathrm{C}$ & 15.822 & 13.468 & 10.481 \\
\hline $\mathrm{O}$ & 13.881 & 12.078 & 13.780 & $\mathrm{O}$ & 14.118 & 12.196 & 14.009 \\
\hline $\mathrm{N}$ & 15.211 & 11.778 & 12.010 & $\mathrm{~N}$ & 15.422 & 11.842 & 12.161 \\
\hline $\mathrm{N}$ & 15.607 & 12.147 & 10.729 & $\mathrm{~N}$ & 15.996 & 12.243 & 10.990 \\
\hline $\mathrm{N}$ & 13.100 & 14.561 & 14.407 & $\mathrm{~N}$ & 13.138 & 14.573 & 14.453 \\
\hline $\mathrm{H}$ & 12.436 & 15.175 & 14.844 & $\mathrm{H}$ & 12.175 & 14.845 & 14.587 \\
\hline $\mathrm{H}$ & 13.071 & 13.577 & 14.638 & $\mathrm{H}$ & 13.313 & 13.549 & 14.562 \\
\hline $\mathrm{O}$ & 16.184 & 13.768 & 9.290 & $\mathrm{O}$ & 16.349 & 13.839 & 9.377 \\
\hline $\mathrm{H}$ & 14.975 & 10.798 & 12.085 & & & & \\
\hline $\mathrm{H}$ & 16.397 & 11.618 & 10.389 & & & & \\
\hline
\end{tabular}




\begin{tabular}{|c|c|c|c|c|c|c|c|}
\hline \multicolumn{8}{|c|}{ 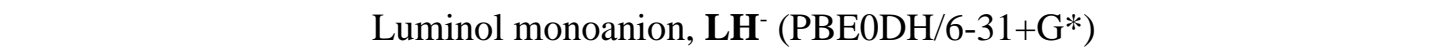 } \\
\hline \multicolumn{4}{|c|}{$1-\mathrm{C}$} & \multicolumn{4}{|c|}{$4-\mathrm{C}$} \\
\hline $\mathrm{C}$ & 13.580 & 14.918 & 13.188 & $\mathrm{C}$ & 13.625 & 14.906 & 13.183 \\
\hline $\mathrm{C}$ & 14.338 & 13.992 & 12.429 & $\mathrm{C}$ & 14.364 & 13.976 & 12.400 \\
\hline $\mathrm{C}$ & 14.918 & 14.380 & 11.211 & $\mathrm{C}$ & 14.883 & 14.410 & 11.172 \\
\hline $\mathrm{C}$ & 14.740 & 15.680 & 10.734 & $\mathrm{C}$ & 14.681 & 15.707 & 10.692 \\
\hline $\mathrm{C}$ & 13.980 & 16.575 & 11.466 & $\mathrm{C}$ & 13.934 & 16.587 & 11.451 \\
\hline $\mathrm{C}$ & 13.409 & 16.206 & 12.682 & $\mathrm{C}$ & 13.417 & 16.194 & 12.682 \\
\hline $\mathrm{H}$ & 15.207 & 15.936 & 9.792 & $\mathrm{H}$ & 15.111 & 15.976 & 9.736 \\
\hline $\mathrm{H}$ & 13.829 & 17.586 & 11.099 & $\mathrm{H}$ & 13.754 & 17.597 & 11.097 \\
\hline $\mathrm{H}$ & 12.836 & 16.926 & 13.259 & $\mathrm{H}$ & 12.852 & 16.900 & 13.285 \\
\hline $\mathrm{C}$ & 14.493 & 12.605 & 12.850 & $\mathrm{C}$ & 14.539 & 12.560 & 12.827 \\
\hline $\mathrm{C}$ & 15.730 & 13.427 & 10.398 & $\mathrm{C}$ & 15.666 & 13.475 & 10.360 \\
\hline $\mathrm{O}$ & 13.981 & 12.094 & 13.881 & $\mathrm{O}$ & 13.999 & 12.147 & 13.897 \\
\hline $\mathrm{N}$ & 15.238 & 11.869 & 12.018 & $\mathrm{~N}$ & 15.250 & 11.738 & 12.067 \\
\hline $\mathrm{N}$ & 15.869 & 12.175 & 10.838 & $\mathrm{~N}$ & 15.773 & 12.256 & 10.911 \\
\hline $\mathrm{N}$ & 13.076 & 14.568 & 14.426 & $\mathrm{~N}$ & 13.172 & 14.558 & 14.434 \\
\hline $\mathrm{H}$ & 12.216 & 15.025 & 14.681 & $\mathrm{H}$ & 12.327 & 15.016 & 14.733 \\
\hline $\mathrm{H}$ & 13.103 & 13.562 & 14.583 & $\mathrm{H}$ & 13.234 & 13.541 & 14.574 \\
\hline $\mathrm{O}$ & 16.251 & 13.830 & 9.333 & $\mathrm{O}$ & 16.195 & 13.757 & 9.268 \\
\hline $\mathrm{H}$ & 15.349 & 10.904 & 12.287 & $\mathrm{H}$ & 16.304 & 11.585 & 10.379 \\
\hline
\end{tabular}




\begin{tabular}{|c|c|c|c|c|c|c|c|}
\hline \multicolumn{8}{|c|}{ LHOOH (PBE1PBE/6-31+G*) } \\
\hline \multicolumn{4}{|c|}{$1-\mathrm{C}$} & \multicolumn{4}{|c|}{$4-\mathrm{C}$} \\
\hline $\mathrm{C}$ & -1.899 & 0.060 & 0.050 & $\mathrm{C}$ & -1.969 & -0.115 & 0.182 \\
\hline $\mathrm{C}$ & -0.538 & -0.078 & -0.129 & $\mathrm{C}$ & -0.583 & 0.008 & -0.041 \\
\hline $\mathrm{C}$ & 0.063 & -1.336 & -0.301 & $\mathrm{C}$ & -0.007 & 1.275 & -0.193 \\
\hline $\mathrm{C}$ & -0.731 & -2.514 & -0.251 & $\mathrm{C}$ & -0.765 & 2.441 & -0.146 \\
\hline $\mathrm{C}$ & -2.125 & -2.357 & -0.084 & $\mathrm{C}$ & -2.138 & 2.322 & 0.055 \\
\hline $\mathrm{C}$ & -2.686 & -1.102 & 0.055 & $\mathrm{C}$ & -2.724 & 1.074 & 0.212 \\
\hline $\mathrm{H}$ & -2.755 & -3.243 & -0.054 & $\mathrm{H}$ & -0.280 & 3.404 & -0.269 \\
\hline $\mathrm{H}$ & -3.762 & -1.017 & 0.184 & $\mathrm{H}$ & -2.762 & 3.210 & 0.092 \\
\hline $\mathrm{C}$ & 0.395 & 1.089 & -0.057 & $\mathrm{H}$ & -3.798 & 1.002 & 0.375 \\
\hline $\mathrm{C}$ & 1.477 & -1.388 & -0.601 & $\mathrm{C}$ & 0.381 & -1.135 & -0.026 \\
\hline $\mathrm{O}$ & 1.640 & 2.273 & 1.489 & $\mathrm{C}$ & 1.442 & 1.345 & -0.436 \\
\hline $\mathrm{O}$ & -0.199 & 2.249 & -0.481 & $\mathrm{O}$ & 1.750 & -2.301 & 1.426 \\
\hline $\mathrm{N}$ & 1.647 & 0.947 & -0.864 & $\mathrm{O}$ & -0.174 & -2.320 & -0.466 \\
\hline $\mathrm{N}$ & 2.135 & -0.163 & -1.084 & $\mathrm{~N}$ & 1.588 & -0.943 & -0.899 \\
\hline $\mathrm{O}$ & 0.790 & 1.132 & 1.309 & $\mathrm{~N}$ & 2.070 & 0.177 & -1.070 \\
\hline $\mathrm{O}$ & 2.174 & -2.388 & -0.594 & $\mathrm{~N}$ & -2.578 & -1.322 & 0.423 \\
\hline $\mathrm{H}$ & 1.050 & 2.846 & 2.006 & $\mathrm{H}$ & -3.581 & -1.348 & 0.313 \\
\hline $\mathrm{H}$ & 0.524 & 2.854 & -0.722 & $\mathrm{H}$ & -2.096 & -2.147 & 0.091 \\
\hline $\mathrm{N}$ & -0.174 & -3.739 & -0.369 & $\mathrm{O}$ & 0.830 & -1.203 & 1.316 \\
\hline $\mathrm{H}$ & 0.834 & -3.819 & -0.438 & $\mathrm{O}$ & 2.147 & 2.302 & -0.233 \\
\hline $\mathrm{H}$ & -0.747 & -4.566 & -0.384 & $\mathrm{H}$ & 1.231 & -2.902 & 1.986 \\
\hline $\mathrm{H}$ & -2.340 & 1.042 & 0.180 & $\mathrm{H}$ & 0.563 & -2.857 & -0.805 \\
\hline
\end{tabular}




\begin{tabular}{|c|c|c|c|c|c|c|c|}
\hline \multicolumn{8}{|c|}{ L-OOH (PBE1PBE/6-31+G*) } \\
\hline \multicolumn{4}{|c|}{$1-\mathrm{C}$} & \multicolumn{4}{|c|}{$4-\mathrm{C}$} \\
\hline $\mathrm{C}$ & -1.647 & 0.193 & 0.357 & $\mathrm{C}$ & -1.760 & -0.228 & 0.088 \\
\hline $\mathrm{C}$ & -0.302 & -0.002 & 0.071 & $\mathrm{C}$ & -0.362 & -0.077 & -0.087 \\
\hline $\mathrm{C}$ & 0.179 & -1.276 & -0.270 & $\mathrm{C}$ & 0.170 & 1.201 & -0.240 \\
\hline $\mathrm{C}$ & -0.716 & -2.379 & -0.361 & $\mathrm{C}$ & -0.629 & 2.353 & -0.214 \\
\hline $\mathrm{C}$ & -2.068 & -2.163 & -0.055 & $\mathrm{C}$ & -1.996 & 2.200 & -0.036 \\
\hline $\mathrm{C}$ & -2.516 & -0.896 & 0.301 & $\mathrm{C}$ & -2.553 & 0.929 & 0.110 \\
\hline $\mathrm{H}$ & -2.766 & -2.997 & -0.120 & $\mathrm{H}$ & -0.165 & 3.326 & -0.334 \\
\hline $\mathrm{H}$ & -3.571 & -0.756 & 0.532 & $\mathrm{H}$ & -2.647 & 3.072 & -0.012 \\
\hline $\mathrm{C}$ & 0.649 & 1.171 & 0.006 & $\mathrm{H}$ & -3.629 & 0.822 & 0.251 \\
\hline $\mathrm{C}$ & 1.615 & -1.435 & -0.450 & $\mathrm{C}$ & 0.555 & -1.278 & -0.122 \\
\hline $\mathrm{O}$ & 0.794 & 3.250 & 1.043 & $\mathrm{C}$ & 1.615 & 1.316 & -0.478 \\
\hline $\mathrm{O}$ & 0.521 & 1.924 & -1.050 & $\mathrm{O}$ & 1.328 & -2.842 & 1.436 \\
\hline $\mathrm{N}$ & 2.100 & 0.798 & 0.101 & $\mathrm{O}$ & 0.063 & -2.359 & -0.666 \\
\hline $\mathrm{N}$ & 2.526 & -0.345 & -0.153 & $\mathrm{~N}$ & 1.906 & -0.997 & -0.759 \\
\hline $\mathrm{O}$ & 0.405 & 1.890 & 1.267 & $\mathrm{~N}$ & 2.360 & 0.147 & -0.927 \\
\hline $\mathrm{O}$ & 2.150 & -2.487 & -0.797 & $\mathrm{~N}$ & -2.300 & -1.483 & 0.265 \\
\hline $\mathrm{H}$ & 0.749 & 3.229 & 0.041 & $\mathrm{H}$ & -3.262 & -1.581 & -0.028 \\
\hline $\mathrm{N}$ & -0.282 & -3.614 & -0.772 & $\mathrm{H}$ & -1.652 & -2.202 & -0.089 \\
\hline $\mathrm{H}$ & -0.825 & -4.413 & -0.484 & $\mathrm{O}$ & 0.925 & -1.472 & 1.303 \\
\hline $\mathrm{H}$ & 0.729 & -3.722 & -0.816 & $\mathrm{O}$ & 2.240 & 2.355 & -0.377 \\
\hline $\mathrm{H}$ & -1.992 & 1.187 & 0.619 & $\mathrm{H}$ & 0.864 & -3.192 & 0.622 \\
\hline
\end{tabular}




\begin{tabular}{|c|c|c|c|c|c|c|c|}
\hline \multicolumn{8}{|c|}{ LHOO- $^{-(P B E 1 P B E / 6-31+G *) ~}$} \\
\hline \multicolumn{4}{|c|}{$1-\mathrm{C}$} & \multicolumn{4}{|c|}{$4-\mathrm{C}$} \\
\hline $\mathrm{C}$ & -1.094 & -1.110 & 1.024 & $\mathrm{C}$ & -0.997 & 1.155 & 1.065 \\
\hline $\mathrm{C}$ & -0.391 & -0.243 & 0.197 & $\mathrm{C}$ & -0.316 & 0.233 & 0.244 \\
\hline $\mathrm{C}$ & -0.047 & -0.597 & -1.110 & $\mathrm{C}$ & -0.055 & 0.552 & -1.086 \\
\hline $\mathrm{C}$ & -0.423 & -1.871 & -1.614 & $\mathrm{C}$ & -0.487 & 1.757 & -1.653 \\
\hline $\mathrm{C}$ & -1.149 & -2.731 & -0.774 & $\mathrm{C}$ & -1.202 & 2.643 & -0.859 \\
\hline $\mathrm{C}$ & -1.475 & -2.353 & 0.522 & $\mathrm{C}$ & -1.442 & 2.351 & 0.483 \\
\hline $\mathrm{H}$ & -1.441 & -3.711 & -1.151 & $\mathrm{H}$ & -0.259 & 1.964 & -2.694 \\
\hline $\mathrm{H}$ & -2.034 & -3.043 & 1.152 & $\mathrm{H}$ & -1.562 & 3.583 & -1.274 \\
\hline $\mathrm{C}$ & 0.000 & 1.125 & 0.688 & $\mathrm{H}$ & -1.966 & 3.071 & 1.111 \\
\hline $\mathrm{C}$ & 0.699 & 0.378 & -1.922 & $\mathrm{C}$ & 0.043 & -1.162 & 0.685 \\
\hline $\mathrm{O}$ & 1.936 & 0.785 & 1.425 & $\mathrm{C}$ & 0.730 & -0.402 & -1.893 \\
\hline $\mathrm{O}$ & -1.158 & 1.913 & 0.718 & $\mathrm{O}$ & 1.711 & -0.619 & 1.884 \\
\hline $\mathrm{N}$ & 1.015 & 1.836 & -0.126 & $\mathrm{O}$ & -1.072 & -1.988 & 0.437 \\
\hline $\mathrm{N}$ & 1.222 & 1.554 & -1.347 & $\mathrm{~N}$ & 1.194 & -1.765 & -0.050 \\
\hline $\mathrm{O}$ & 0.613 & 1.103 & 1.921 & $\mathrm{~N}$ & 1.441 & -1.467 & -1.245 \\
\hline $\mathrm{O}$ & 0.895 & 0.209 & -3.132 & $\mathrm{~N}$ & -1.180 & 0.926 & 2.417 \\
\hline $\mathrm{H}$ & -0.833 & 2.796 & 0.962 & $\mathrm{H}$ & -2.075 & 1.194 & 2.800 \\
\hline $\mathrm{N}$ & -0.047 & -2.277 & -2.873 & $\mathrm{H}$ & -0.814 & 0.029 & 2.730 \\
\hline $\mathrm{H}$ & 0.269 & -1.516 & -3.472 & $\mathrm{O}$ & 0.417 & -1.274 & 2.000 \\
\hline $\mathrm{H}$ & -0.604 & -3.001 & -3.300 & $\mathrm{O}$ & 0.838 & -0.310 & -3.106 \\
\hline $\mathrm{H}$ & -1.323 & -0.811 & 2.041 & $\mathrm{H}$ & -0.769 & -2.879 & 0.680 \\
\hline
\end{tabular}




\begin{tabular}{|c|c|c|c|c|c|c|c|}
\hline \multicolumn{8}{|c|}{ LHOOH (M062X/6-31+G*) } \\
\hline \multicolumn{4}{|c|}{$1-\mathrm{C}$} & \multicolumn{4}{|c|}{$4-\mathrm{C}$} \\
\hline $\mathrm{C}$ & 13.499 & 14.875 & 13.196 & $\mathrm{C}$ & 13.871 & 14.865 & 13.180 \\
\hline $\mathrm{C}$ & 14.324 & 13.953 & 12.508 & $\mathrm{C}$ & 14.548 & 14.051 & 12.253 \\
\hline $\mathrm{C}$ & 15.038 & 14.357 & 11.370 & $\mathrm{C}$ & 14.826 & 14.533 & 10.972 \\
\hline $\mathrm{C}$ & 14.941 & 15.634 & 10.860 & $\mathrm{C}$ & 14.434 & 15.798 & 10.552 \\
\hline $\mathrm{C}$ & 14.092 & 16.538 & 11.521 & $\mathrm{C}$ & 13.740 & 16.597 & 11.459 \\
\hline $\mathrm{C}$ & 13.395 & 16.179 & 12.659 & $\mathrm{C}$ & 13.463 & 16.140 & 12.740 \\
\hline $\mathrm{H}$ & 15.504 & 15.920 & 9.979 & $\mathrm{H}$ & 14.670 & 16.132 & 9.548 \\
\hline $\mathrm{H}$ & 13.987 & 17.549 & 11.138 & $\mathrm{H}$ & 13.411 & 17.590 & 11.168 \\
\hline $\mathrm{H}$ & 12.759 & 16.903 & 13.161 & $\mathrm{H}$ & 12.930 & 16.781 & 13.438 \\
\hline $\mathrm{C}$ & 14.404 & 12.552 & 12.897 & $\mathrm{C}$ & 15.126 & 12.706 & 12.571 \\
\hline $\mathrm{C}$ & 15.987 & 13.340 & 10.815 & $\mathrm{C}$ & 15.545 & 13.635 & 10.043 \\
\hline $\mathrm{O}$ & 17.125 & 13.422 & 11.661 & $\mathrm{O}$ & 17.064 & 11.719 & 13.311 \\
\hline $\mathrm{O}$ & 14.058 & 12.083 & 13.959 & $\mathrm{O}$ & 14.412 & 12.024 & 13.534 \\
\hline $\mathrm{N}$ & 14.806 & 11.571 & 11.859 & $\mathrm{~N}$ & 15.186 & 11.757 & 11.399 \\
\hline $\mathrm{N}$ & 15.489 & 11.923 & 10.899 & $\mathrm{~N}$ & 15.396 & 12.179 & 10.264 \\
\hline $\mathrm{N}$ & 12.811 & 14.521 & 14.310 & $\mathrm{~N}$ & 13.657 & 14.489 & 14.490 \\
\hline $\mathrm{H}$ & 12.256 & 15.206 & 14.799 & $\mathrm{H}$ & 12.956 & 15.016 & 14.991 \\
\hline $\mathrm{H}$ & 12.997 & 13.632 & 14.755 & $\mathrm{H}$ & 13.651 & 13.496 & 14.683 \\
\hline $\mathrm{O}$ & 18.097 & 12.504 & 11.163 & $\mathrm{O}$ & 16.460 & 12.967 & 12.965 \\
\hline $\mathrm{O}$ & 16.286 & 13.575 & 9.499 & $\mathrm{O}$ & 16.171 & 13.980 & 9.079 \\
\hline $\mathrm{H}$ & 16.596 & 12.733 & 9.123 & $\mathrm{H}$ & 14.603 & 11.077 & 13.408 \\
\hline $\mathrm{H}$ & 18.763 & 13.120 & 10.813 & $\mathrm{H}$ & 17.199 & 11.847 & 14.265 \\
\hline
\end{tabular}




\begin{tabular}{|c|c|c|c|c|c|c|c|}
\hline \multicolumn{8}{|c|}{ L-OOH (M062X/6-31+G*) } \\
\hline \multicolumn{4}{|c|}{$1-\mathrm{C}$} & \multicolumn{4}{|c|}{$4-\mathrm{C}$} \\
\hline $\mathrm{C}$ & 13.538 & 14.905 & 13.232 & $\mathrm{C}$ & 13.862 & 14.834 & 13.143 \\
\hline $\mathrm{C}$ & 14.365 & 13.964 & 12.573 & $\mathrm{C}$ & 14.626 & 14.073 & 12.229 \\
\hline $\mathrm{C}$ & 15.082 & 14.322 & 11.426 & $\mathrm{C}$ & 14.923 & 14.611 & 10.981 \\
\hline $\mathrm{C}$ & 14.963 & 15.590 & 10.879 & $\mathrm{C}$ & 14.482 & 15.874 & 10.577 \\
\hline $\mathrm{C}$ & 14.114 & 16.508 & 11.501 & $\mathrm{C}$ & 13.709 & 16.608 & 11.468 \\
\hline $\mathrm{C}$ & 13.419 & 16.182 & 12.661 & $\mathrm{C}$ & 13.404 & 16.096 & 12.729 \\
\hline $\mathrm{H}$ & 15.530 & 15.819 & 9.983 & $\mathrm{H}$ & 14.746 & 16.246 & 9.592 \\
\hline $\mathrm{H}$ & 13.999 & 17.507 & 11.084 & $\mathrm{H}$ & 13.340 & 17.593 & 11.190 \\
\hline $\mathrm{H}$ & 12.790 & 16.922 & 13.153 & $\mathrm{H}$ & 12.811 & 16.687 & 13.425 \\
\hline $\mathrm{C}$ & 14.426 & 12.558 & 12.971 & $\mathrm{C}$ & 15.171 & 12.698 & 12.579 \\
\hline $\mathrm{C}$ & 16.041 & 13.312 & 10.820 & $\mathrm{C}$ & 15.703 & 13.757 & 10.061 \\
\hline $\mathrm{O}$ & 17.145 & 13.315 & 11.822 & $\mathrm{O}$ & 17.070 & 11.830 & 13.596 \\
\hline $\mathrm{O}$ & 14.069 & 12.120 & 14.052 & $\mathrm{O}$ & 14.543 & 12.019 & 13.490 \\
\hline $\mathrm{N}$ & 14.777 & 11.559 & 11.948 & $\mathrm{~N}$ & 15.316 & 11.812 & 11.310 \\
\hline $\mathrm{N}$ & 15.474 & 11.868 & 10.975 & $\mathrm{~N}$ & 15.566 & 12.304 & 10.205 \\
\hline $\mathrm{N}$ & 12.896 & 14.605 & 14.420 & $\mathrm{~N}$ & 13.620 & 14.366 & 14.420 \\
\hline $\mathrm{H}$ & 12.020 & 15.080 & 14.585 & $\mathrm{H}$ & 12.764 & 14.699 & 14.844 \\
\hline $\mathrm{H}$ & 12.927 & 13.629 & 14.697 & $\mathrm{H}$ & 13.783 & 13.352 & 14.478 \\
\hline $\mathrm{O}$ & 18.280 & 12.765 & 11.152 & $\mathrm{O}$ & 16.572 & 12.994 & 12.932 \\
\hline $\mathrm{O}$ & 16.435 & 13.519 & 9.618 & $\mathrm{O}$ & 16.362 & 14.171 & 9.134 \\
\hline $\mathrm{H}$ & 18.012 & 12.992 & 10.219 & $\mathrm{H}$ & 16.212 & 11.500 & 13.971 \\
\hline
\end{tabular}




\begin{tabular}{|c|c|c|c|c|c|c|c|}
\hline \multicolumn{8}{|c|}{ LHOO- $^{-}\left(\mathrm{M} 062 \mathrm{X} / 6-31+\mathrm{G}^{*}\right)$} \\
\hline \multicolumn{4}{|c|}{$1-\mathrm{C}$} & \multicolumn{4}{|c|}{$4-\mathrm{C}$} \\
\hline $\mathrm{C}$ & -0.919 & 1.126 & -1.063 & $\mathrm{C}$ & -0.996 & 1.126 & 1.084 \\
\hline $\mathrm{C}$ & -0.296 & 0.243 & -0.192 & $\mathrm{C}$ & -0.348 & 0.197 & 0.232 \\
\hline $\mathrm{C}$ & -0.044 & 0.592 & 1.136 & $\mathrm{C}$ & -0.129 & 0.526 & -1.104 \\
\hline $\mathrm{C}$ & -0.442 & 1.857 & 1.634 & $\mathrm{C}$ & -0.572 & 1.719 & -1.679 \\
\hline $\mathrm{C}$ & -1.078 & 2.737 & 0.750 & $\mathrm{C}$ & -1.263 & 2.606 & -0.861 \\
\hline $\mathrm{C}$ & -1.302 & 2.372 & -0.577 & $\mathrm{C}$ & -1.463 & 2.317 & 0.485 \\
\hline $\mathrm{H}$ & -1.385 & 3.718 & 1.109 & $\mathrm{H}$ & -0.370 & 1.923 & -2.725 \\
\hline $\mathrm{H}$ & -1.783 & 3.086 & -1.242 & $\mathrm{H}$ & -1.640 & 3.543 & -1.265 \\
\hline $\mathrm{C}$ & 0.031 & -1.162 & -0.631 & $\mathrm{H}$ & -1.983 & 3.035 & 1.118 \\
\hline $\mathrm{C}$ & 0.674 & -0.384 & 1.954 & $\mathrm{C}$ & 0.037 & -1.223 & 0.591 \\
\hline $\mathrm{O}$ & 1.502 & -0.440 & -2.062 & $\mathrm{C}$ & 0.684 & -0.424 & -1.892 \\
\hline $\mathrm{O}$ & -1.067 & -1.983 & -0.295 & $\mathrm{O}$ & 0.919 & -0.668 & 2.603 \\
\hline $\mathrm{N}$ & 1.198 & -1.768 & 0.122 & $\mathrm{O}$ & -0.860 & -2.084 & -0.077 \\
\hline $\mathrm{N}$ & 1.426 & -1.458 & 1.299 & $\mathrm{~N}$ & 1.410 & -1.576 & 0.010 \\
\hline $\mathrm{O}$ & 0.298 & -1.267 & -1.948 & $\mathrm{~N}$ & 1.664 & -1.242 & -1.151 \\
\hline $\mathrm{O}$ & 0.754 & -0.346 & 3.171 & $\mathrm{~N}$ & -1.167 & 0.872 & 2.419 \\
\hline $\mathrm{H}$ & -0.877 & -2.830 & -0.732 & $\mathrm{H}$ & -1.390 & 1.699 & 2.960 \\
\hline $\mathrm{H}$ & -1.053 & 0.829 & -2.097 & $\mathrm{H}$ & -0.350 & 0.289 & 2.789 \\
\hline $\mathrm{N}$ & -0.164 & 2.239 & 2.936 & $\mathrm{O}$ & 0.031 & -1.574 & 1.902 \\
\hline $\mathrm{H}$ & 0.024 & 1.477 & 3.577 & $\mathrm{O}$ & 0.685 & -0.516 & -3.097 \\
\hline $\mathrm{H}$ & -0.739 & 2.984 & 3.304 & $\mathrm{H}$ & -0.722 & -2.954 & 0.333 \\
\hline
\end{tabular}




\begin{tabular}{|c|c|c|c|c|c|c|c|}
\hline \multicolumn{8}{|c|}{ LHOOH (CAM-B3LYP/6-31+G*) } \\
\hline \multicolumn{4}{|c|}{$1-\mathrm{C}$} & \multicolumn{4}{|c|}{$4-\mathrm{C}$} \\
\hline $\mathrm{C}$ & 13.488 & 14.876 & 13.195 & $\mathrm{C}$ & 13.885 & 14.867 & 13.178 \\
\hline $\mathrm{C}$ & 14.306 & 13.941 & 12.514 & $\mathrm{C}$ & 14.554 & 14.051 & 12.248 \\
\hline $\mathrm{C}$ & 15.045 & 14.342 & 11.390 & $\mathrm{C}$ & 14.816 & 14.529 & 10.962 \\
\hline $\mathrm{C}$ & 14.973 & 15.624 & 10.893 & $\mathrm{C}$ & 14.422 & 15.795 & 10.550 \\
\hline $\mathrm{C}$ & 14.130 & 16.537 & 11.545 & $\mathrm{C}$ & 13.742 & 16.596 & 11.462 \\
\hline $\mathrm{C}$ & 13.412 & 16.183 & 12.667 & $\mathrm{C}$ & 13.478 & 16.141 & 12.743 \\
\hline $\mathrm{H}$ & 15.551 & 15.911 & 10.024 & $\mathrm{H}$ & 14.646 & 16.129 & 9.544 \\
\hline $\mathrm{H}$ & 14.049 & 17.552 & 11.166 & $\mathrm{H}$ & 13.414 & 17.590 & 11.173 \\
\hline $\mathrm{H}$ & 12.781 & 16.914 & 13.165 & $\mathrm{H}$ & 12.953 & 16.784 & 13.445 \\
\hline $\mathrm{C}$ & 14.362 & 12.547 & 12.907 & $\mathrm{C}$ & 15.125 & 12.704 & 12.565 \\
\hline $\mathrm{C}$ & 15.986 & 13.324 & 10.824 & $\mathrm{C}$ & 15.521 & 13.634 & 10.026 \\
\hline $\mathrm{O}$ & 17.167 & 13.468 & 11.608 & $\mathrm{O}$ & 17.067 & 11.717 & 13.354 \\
\hline $\mathrm{O}$ & 13.957 & 12.084 & 13.956 & $\mathrm{O}$ & 14.394 & 12.016 & 13.514 \\
\hline $\mathrm{N}$ & 14.835 & 11.559 & 11.919 & $\mathrm{~N}$ & 15.203 & 11.763 & 11.397 \\
\hline $\mathrm{N}$ & 15.542 & 11.903 & 10.970 & $\mathrm{~N}$ & 15.396 & 12.186 & 10.258 \\
\hline $\mathrm{N}$ & 12.790 & 14.530 & 14.304 & $\mathrm{~N}$ & 13.668 & 14.486 & 14.484 \\
\hline $\mathrm{H}$ & 12.203 & 15.203 & 14.769 & $\mathrm{H}$ & 12.977 & 15.013 & 14.998 \\
\hline $\mathrm{H}$ & 12.924 & 13.614 & 14.712 & $\mathrm{H}$ & 13.675 & 13.494 & 14.679 \\
\hline $\mathrm{O}$ & 18.161 & 12.571 & 11.084 & $\mathrm{O}$ & 16.456 & 12.967 & 12.986 \\
\hline $\mathrm{O}$ & 16.209 & 13.520 & 9.485 & $\mathrm{O}$ & 16.132 & 13.980 & 9.049 \\
\hline $\mathrm{H}$ & 16.547 & 12.680 & 9.127 & $\mathrm{H}$ & 14.588 & 11.070 & 13.391 \\
\hline $\mathrm{H}$ & 18.797 & 13.210 & 10.719 & $\mathrm{H}$ & 17.209 & 11.867 & 14.305 \\
\hline
\end{tabular}




\begin{tabular}{|c|c|c|c|c|c|c|c|}
\hline \multicolumn{8}{|c|}{ L-OOH (CAM-B3LYP/6-31+G*) } \\
\hline \multicolumn{4}{|c|}{$1-\mathrm{C}$} & \multicolumn{4}{|c|}{$4-\mathrm{C}$} \\
\hline $\mathrm{C}$ & 13.400 & 14.866 & 13.176 & $\mathrm{C}$ & 13.908 & 14.831 & 13.142 \\
\hline $\mathrm{C}$ & 14.325 & 13.922 & 12.662 & $\mathrm{C}$ & 14.645 & 14.069 & 12.206 \\
\hline $\mathrm{C}$ & 15.261 & 14.301 & 11.693 & $\mathrm{C}$ & 14.896 & 14.602 & 10.947 \\
\hline $\mathrm{C}$ & 15.313 & 15.611 & 11.237 & $\mathrm{C}$ & 14.443 & 15.868 & 10.564 \\
\hline $\mathrm{C}$ & 14.417 & 16.541 & 11.754 & $\mathrm{C}$ & 13.710 & 16.606 & 11.480 \\
\hline $\mathrm{C}$ & 13.471 & 16.184 & 12.706 & $\mathrm{C}$ & 13.446 & 16.094 & 12.748 \\
\hline $\mathrm{H}$ & 16.044 & 15.879 & 10.484 & $\mathrm{H}$ & 14.671 & 16.239 & 9.570 \\
\hline $\mathrm{H}$ & 14.450 & 17.571 & 11.405 & $\mathrm{H}$ & 13.337 & 17.592 & 11.214 \\
\hline $\mathrm{H}$ & 12.778 & 16.924 & 13.100 & $\mathrm{H}$ & 12.877 & 16.686 & 13.463 \\
\hline $\mathrm{C}$ & 14.325 & 12.527 & 13.088 & $\mathrm{C}$ & 15.190 & 12.697 & 12.549 \\
\hline $\mathrm{C}$ & 16.112 & 13.226 & 11.048 & $\mathrm{C}$ & 15.641 & 13.760 & 9.996 \\
\hline $\mathrm{O}$ & 17.463 & 13.812 & 11.030 & $\mathrm{O}$ & 17.004 & 11.864 & 13.779 \\
\hline $\mathrm{O}$ & 13.441 & 12.014 & 13.764 & $\mathrm{O}$ & 14.522 & 12.000 & 13.424 \\
\hline $\mathrm{N}$ & 15.458 & 11.667 & 12.775 & $\mathrm{~N}$ & 15.417 & 11.828 & 11.304 \\
\hline $\mathrm{N}$ & 16.288 & 11.996 & 11.911 & $\mathrm{~N}$ & 15.615 & 12.313 & 10.181 \\
\hline $\mathrm{N}$ & 12.485 & 14.518 & 14.148 & $\mathrm{~N}$ & 13.685 & 14.348 & 14.416 \\
\hline $\mathrm{H}$ & 11.680 & 15.118 & 14.247 & $\mathrm{H}$ & 12.853 & 14.694 & 14.873 \\
\hline $\mathrm{H}$ & 12.329 & 13.521 & 14.256 & $\mathrm{H}$ & 13.832 & 13.331 & 14.455 \\
\hline $\mathrm{O}$ & 18.178 & 13.169 & 9.962 & $\mathrm{O}$ & 16.579 & 12.992 & 12.993 \\
\hline $\mathrm{O}$ & 15.706 & 12.832 & 9.875 & $\mathrm{O}$ & 16.216 & 14.183 & 9.015 \\
\hline $\mathrm{H}$ & 17.380 & 12.842 & 9.451 & $\mathrm{H}$ & 16.098 & 11.531 & 14.035 \\
\hline
\end{tabular}




\begin{tabular}{|c|c|c|c|c|c|c|c|}
\hline \multicolumn{8}{|c|}{ LHOO- $^{-}$(CAM-B3LYP/6-31+G*) } \\
\hline \multicolumn{4}{|c|}{$1-\mathrm{C}$} & \multicolumn{4}{|c|}{$4-\mathrm{C}$} \\
\hline $\mathrm{C}$ & -0.991 & 1.110 & -1.043 & $\mathrm{C}$ & -0.985 & 1.141 & 1.057 \\
\hline $\mathrm{C}$ & -0.328 & 0.241 & -0.193 & $\mathrm{C}$ & -0.295 & 0.228 & 0.229 \\
\hline $\mathrm{C}$ & -0.041 & 0.597 & 1.126 & $\mathrm{C}$ & -0.047 & 0.561 & -1.097 \\
\hline $\mathrm{C}$ & -0.438 & 1.864 & 1.622 & $\mathrm{C}$ & -0.487 & 1.764 & -1.659 \\
\hline $\mathrm{C}$ & -1.114 & 2.731 & 0.754 & $\mathrm{C}$ & -1.201 & 2.640 & -0.857 \\
\hline $\mathrm{C}$ & -1.378 & 2.354 & -0.557 & $\mathrm{C}$ & -1.438 & 2.335 & 0.481 \\
\hline $\mathrm{H}$ & -1.419 & 3.710 & 1.117 & $\mathrm{H}$ & -0.268 & 1.978 & -2.699 \\
\hline $\mathrm{H}$ & -1.896 & 3.051 & -1.212 & $\mathrm{H}$ & -1.565 & 3.580 & -1.263 \\
\hline $\mathrm{C}$ & 0.027 & -1.150 & -0.655 & $\mathrm{H}$ & -1.964 & 3.047 & 1.114 \\
\hline $\mathrm{C}$ & 0.689 & -0.366 & 1.945 & $\mathrm{C}$ & 0.062 & -1.172 & 0.665 \\
\hline $\mathrm{O}$ & 1.746 & -0.548 & -1.783 & $\mathrm{C}$ & 0.717 & -0.393 & -1.917 \\
\hline $\mathrm{O}$ & -1.118 & -1.960 & -0.478 & $\mathrm{O}$ & 1.623 & -0.524 & 2.052 \\
\hline $\mathrm{N}$ & 1.108 & -1.812 & 0.143 & $\mathrm{O}$ & -1.045 & -2.004 & 0.372 \\
\hline $\mathrm{N}$ & 1.330 & -1.508 & 1.333 & $\mathrm{~N}$ & 1.211 & -1.780 & -0.093 \\
\hline $\mathrm{O}$ & 0.440 & -1.203 & -1.946 & $\mathrm{~N}$ & 1.460 & -1.465 & -1.269 \\
\hline $\mathrm{O}$ & 0.836 & -0.257 & 3.159 & $\mathrm{~N}$ & -1.176 & 0.896 & 2.398 \\
\hline $\mathrm{H}$ & -0.866 & -2.823 & -0.849 & $\mathrm{H}$ & -2.018 & 1.243 & 2.829 \\
\hline $\mathrm{H}$ & -1.169 & 0.810 & -2.069 & $\mathrm{H}$ & -0.785 & 0.023 & 2.741 \\
\hline $\mathrm{N}$ & -0.131 & 2.258 & 2.907 & $\mathrm{O}$ & 0.377 & -1.308 & 1.983 \\
\hline $\mathrm{H}$ & 0.148 & 1.512 & 3.535 & $\mathrm{O}$ & 0.804 & -0.327 & -3.127 \\
\hline $\mathrm{H}$ & -0.676 & 3.011 & 3.298 & $\mathrm{H}$ & -0.805 & -2.873 & 0.738 \\
\hline
\end{tabular}




\begin{tabular}{|c|c|c|c|c|c|c|c|}
\hline \multicolumn{8}{|c|}{ LHOOH (PBE0DH/6-31+G*) } \\
\hline \multicolumn{4}{|c|}{$1-\mathrm{C}$} & \multicolumn{4}{|c|}{$4-\mathrm{C}$} \\
\hline $\mathrm{C}$ & 13.497 & 14.870 & 13.189 & $\mathrm{C}$ & 13.879 & 14.867 & 13.183 \\
\hline $\mathrm{C}$ & 14.319 & 13.947 & 12.504 & $\mathrm{C}$ & 14.552 & 14.051 & 12.261 \\
\hline $\mathrm{C}$ & 15.040 & 14.350 & 11.374 & $\mathrm{C}$ & 14.829 & 14.526 & 10.980 \\
\hline $\mathrm{C}$ & 14.952 & 15.629 & 10.872 & $\mathrm{C}$ & 14.439 & 15.789 & 10.559 \\
\hline $\mathrm{C}$ & 14.107 & 16.531 & 11.529 & $\mathrm{C}$ & 13.750 & 16.589 & 11.462 \\
\hline $\mathrm{C}$ & 13.402 & 16.172 & 12.658 & $\mathrm{C}$ & 13.475 & 16.138 & 12.741 \\
\hline $\mathrm{H}$ & 15.520 & 15.918 & 9.999 & $\mathrm{H}$ & 14.672 & 16.119 & 9.556 \\
\hline $\mathrm{H}$ & 14.010 & 17.541 & 11.150 & $\mathrm{H}$ & 13.424 & 17.580 & 11.169 \\
\hline $\mathrm{H}$ & 12.769 & 16.897 & 13.156 & $\mathrm{H}$ & 12.945 & 16.782 & 13.434 \\
\hline $\mathrm{C}$ & 14.390 & 12.555 & 12.890 & $\mathrm{C}$ & 15.124 & 12.710 & 12.575 \\
\hline $\mathrm{C}$ & 15.987 & 13.338 & 10.823 & $\mathrm{C}$ & 15.531 & 13.624 & 10.057 \\
\hline $\mathrm{O}$ & 17.128 & 13.448 & 11.652 & $\mathrm{O}$ & 17.073 & 11.728 & 13.281 \\
\hline $\mathrm{O}$ & 14.025 & 12.089 & 13.950 & $\mathrm{O}$ & 14.422 & 12.036 & 13.546 \\
\hline $\mathrm{N}$ & 14.812 & 11.584 & 11.877 & $\mathrm{~N}$ & 15.154 & 11.769 & 11.415 \\
\hline $\mathrm{N}$ & 15.508 & 11.933 & 10.925 & $\mathrm{~N}$ & 15.357 & 12.188 & 10.277 \\
\hline $\mathrm{N}$ & 12.802 & 14.511 & 14.289 & $\mathrm{~N}$ & 13.663 & 14.495 & 14.487 \\
\hline $\mathrm{H}$ & 12.270 & 15.195 & 14.795 & $\mathrm{H}$ & 12.952 & 15.009 & 14.979 \\
\hline $\mathrm{H}$ & 12.998 & 13.621 & 14.722 & $\mathrm{H}$ & 13.665 & 13.506 & 14.676 \\
\hline $\mathrm{O}$ & 18.109 & 12.539 & 11.164 & $\mathrm{O}$ & 16.459 & 12.971 & 12.947 \\
\hline $\mathrm{O}$ & 16.264 & 13.556 & 9.503 & $\mathrm{O}$ & 16.169 & 13.959 & 9.095 \\
\hline $\mathrm{H}$ & 16.556 & 12.710 & 9.137 & $\mathrm{H}$ & 14.581 & 11.092 & 13.399 \\
\hline $\mathrm{H}$ & 18.748 & 13.158 & 10.785 & $\mathrm{H}$ & 17.204 & 11.850 & 14.231 \\
\hline
\end{tabular}




\begin{tabular}{|c|c|c|c|c|c|c|c|}
\hline \multicolumn{8}{|c|}{ L-OOH (PBE0DH/6-31+G*) } \\
\hline \multicolumn{4}{|c|}{$1-\mathrm{C}$} & \multicolumn{4}{|c|}{$4-\mathrm{C}$} \\
\hline $\mathrm{C}$ & 13.407 & 14.864 & 13.179 & $\mathrm{C}$ & 13.900 & 14.825 & 13.132 \\
\hline $\mathrm{C}$ & 14.333 & 13.925 & 12.665 & $\mathrm{C}$ & 14.655 & 14.071 & 12.207 \\
\hline $\mathrm{C}$ & 15.265 & 14.305 & 11.696 & $\mathrm{C}$ & 14.912 & 14.602 & 10.951 \\
\hline $\mathrm{C}$ & 15.314 & 15.613 & 11.239 & $\mathrm{C}$ & 14.446 & 15.857 & 10.556 \\
\hline $\mathrm{C}$ & 14.416 & 16.539 & 11.756 & $\mathrm{C}$ & 13.695 & 16.587 & 11.461 \\
\hline $\mathrm{C}$ & 13.474 & 16.179 & 12.709 & $\mathrm{C}$ & 13.426 & 16.078 & 12.728 \\
\hline $\mathrm{H}$ & 16.041 & 15.881 & 10.485 & $\mathrm{H}$ & 14.677 & 16.225 & 9.564 \\
\hline $\mathrm{H}$ & 14.444 & 17.567 & 11.408 & $\mathrm{H}$ & 13.311 & 17.564 & 11.189 \\
\hline $\mathrm{H}$ & 12.782 & 16.917 & 13.103 & $\mathrm{H}$ & 12.845 & 16.666 & 13.432 \\
\hline $\mathrm{C}$ & 14.337 & 12.532 & 13.086 & $\mathrm{C}$ & 15.206 & 12.711 & 12.560 \\
\hline $\mathrm{C}$ & 16.110 & 13.233 & 11.053 & $\mathrm{C}$ & 15.671 & 13.767 & 10.013 \\
\hline $\mathrm{O}$ & 17.443 & 13.822 & 11.011 & $\mathrm{O}$ & 16.973 & 11.888 & 13.817 \\
\hline $\mathrm{O}$ & 13.454 & 12.016 & 13.757 & $\mathrm{O}$ & 14.511 & 12.008 & 13.408 \\
\hline $\mathrm{N}$ & 15.466 & 11.683 & 12.773 & $\mathrm{~N}$ & 15.474 & 11.850 & 11.336 \\
\hline $\mathrm{N}$ & 16.292 & 12.018 & 11.909 & $\mathrm{~N}$ & 15.673 & 12.330 & 10.211 \\
\hline $\mathrm{N}$ & 12.505 & 14.515 & 14.156 & $\mathrm{~N}$ & 13.680 & 14.344 & 14.401 \\
\hline $\mathrm{H}$ & 11.687 & 15.095 & 14.225 & $\mathrm{H}$ & 12.831 & 14.668 & 14.836 \\
\hline $\mathrm{H}$ & 12.350 & 13.519 & 14.243 & $\mathrm{H}$ & 13.829 & 13.329 & 14.427 \\
\hline $\mathrm{O}$ & 18.147 & 13.161 & 9.963 & $\mathrm{O}$ & 16.561 & 13.016 & 13.045 \\
\hline $\mathrm{O}$ & 15.686 & 12.827 & 9.892 & $\mathrm{O}$ & 16.242 & 14.191 & 9.033 \\
\hline $\mathrm{H}$ & 17.350 & 12.831 & 9.464 & $\mathrm{H}$ & 16.067 & 11.549 & 14.047 \\
\hline
\end{tabular}




\begin{tabular}{|c|c|c|c|c|c|c|c|}
\hline \multicolumn{8}{|c|}{ LHOO- $^{-\left(P B E 0 D H / 6-31+G^{*}\right)}$} \\
\hline \multicolumn{4}{|c|}{$1-\mathrm{C}$} & \multicolumn{4}{|c|}{$4-\mathrm{C}$} \\
\hline $\mathrm{C}$ & -1.022 & 1.113 & -1.043 & $\mathrm{C}$ & -0.976 & 1.152 & 1.067 \\
\hline $\mathrm{C}$ & -0.350 & 0.245 & -0.200 & $\mathrm{C}$ & -0.296 & 0.234 & 0.245 \\
\hline $\mathrm{C}$ & -0.045 & 0.597 & 1.113 & $\mathrm{C}$ & -0.056 & 0.552 & -1.085 \\
\hline $\mathrm{C}$ & -0.429 & 1.864 & 1.612 & $\mathrm{C}$ & -0.491 & 1.751 & -1.652 \\
\hline $\mathrm{C}$ & -1.117 & 2.729 & 0.754 & $\mathrm{C}$ & -1.195 & 2.636 & -0.854 \\
\hline $\mathrm{C}$ & -1.403 & 2.355 & -0.551 & $\mathrm{C}$ & -1.422 & 2.344 & 0.487 \\
\hline $\mathrm{H}$ & -1.413 & 3.708 & 1.120 & $\mathrm{H}$ & -0.277 & 1.954 & -2.694 \\
\hline $\mathrm{H}$ & -1.931 & 3.052 & -1.194 & $\mathrm{H}$ & -1.556 & 3.573 & -1.263 \\
\hline $\mathrm{C}$ & 0.012 & -1.137 & -0.661 & $\mathrm{H}$ & -1.936 & 3.062 & 1.118 \\
\hline $\mathrm{C}$ & 0.692 & -0.373 & 1.923 & $\mathrm{C}$ & 0.054 & -1.164 & 0.673 \\
\hline $\mathrm{O}$ & 1.800 & -0.639 & -1.609 & $\mathrm{C}$ & 0.707 & -0.407 & -1.894 \\
\hline $\mathrm{O}$ & -1.129 & -1.938 & -0.533 & $\mathrm{O}$ & 1.615 & -0.539 & 1.978 \\
\hline $\mathrm{N}$ & 1.094 & -1.784 & 0.111 & $\mathrm{O}$ & -1.043 & -1.988 & 0.365 \\
\hline $\mathrm{N}$ & 1.311 & -1.495 & 1.317 & $\mathrm{~N}$ & 1.218 & -1.744 & -0.054 \\
\hline $\mathrm{O}$ & 0.488 & -1.165 & -1.936 & $\mathrm{~N}$ & 1.462 & -1.443 & -1.238 \\
\hline $\mathrm{O}$ & 0.832 & -0.252 & 3.139 & $\mathrm{~N}$ & -1.157 & 0.927 & 2.416 \\
\hline $\mathrm{H}$ & -0.837 & -2.817 & -0.811 & $\mathrm{H}$ & -2.059 & 1.174 & 2.786 \\
\hline $\mathrm{H}$ & -1.218 & 0.815 & -2.064 & $\mathrm{H}$ & -0.785 & 0.037 & 2.727 \\
\hline $\mathrm{N}$ & -0.091 & 2.262 & 2.883 & $\mathrm{O}$ & 0.370 & -1.297 & 1.987 \\
\hline $\mathrm{H}$ & 0.176 & 1.501 & 3.496 & $\mathrm{O}$ & 0.777 & -0.354 & -3.106 \\
\hline $\mathrm{H}$ & -0.666 & 2.980 & 3.289 & $\mathrm{H}$ & -0.778 & -2.869 & 0.667 \\
\hline
\end{tabular}




\begin{tabular}{|c|c|c|c|c|c|c|c|}
\hline \multicolumn{4}{|c|}{ EP (PBE1PBE/6-31+G*) } & \multicolumn{4}{|c|}{$\mathbf{E P}^{2-}\left(\mathrm{PBE} 1 \mathrm{PBE} / 6-31+\mathrm{G}^{*}\right)$} \\
\hline $\mathrm{C}$ & 13.613 & 14.899 & 13.209 & $\mathrm{C}$ & 15.303 & 12.918 & 13.262 \\
\hline $\mathrm{C}$ & 14.525 & 14.082 & 12.522 & $\mathrm{C}$ & 14.583 & 14.092 & 13.541 \\
\hline $\mathrm{C}$ & 15.087 & 14.499 & 11.313 & $\mathrm{C}$ & 15.224 & 15.328 & 13.564 \\
\hline $\mathrm{C}$ & 14.772 & 15.710 & 10.722 & $\mathrm{C}$ & 16.595 & 15.436 & 13.334 \\
\hline $\mathrm{C}$ & 13.855 & 16.521 & 11.395 & $\mathrm{C}$ & 17.320 & 14.273 & 13.072 \\
\hline $\mathrm{C}$ & 13.293 & 16.131 & 12.602 & $\mathrm{C}$ & 16.683 & 13.027 & 13.029 \\
\hline $\mathrm{H}$ & 15.221 & 16.004 & 9.779 & $\mathrm{H}$ & 17.053 & 16.422 & 13.371 \\
\hline $\mathrm{H}$ & 13.573 & 17.481 & 10.970 & $\mathrm{H}$ & 18.395 & 14.328 & 12.886 \\
\hline $\mathrm{H}$ & 12.591 & 16.791 & 13.107 & $\mathrm{H}$ & 17.252 & 12.125 & 12.790 \\
\hline $\mathrm{C}$ & 15.034 & 12.731 & 12.875 & $\mathrm{C}$ & 13.118 & 14.153 & 13.912 \\
\hline $\mathrm{C}$ & 16.021 & 13.468 & 10.786 & $\mathrm{C}$ & 14.325 & 16.491 & 13.920 \\
\hline $\mathrm{O}$ & 17.068 & 13.210 & 11.769 & $\mathrm{O}$ & 13.163 & 16.395 & 12.916 \\
\hline $\mathrm{O}$ & 14.547 & 12.197 & 14.028 & $\mathrm{O}$ & 12.445 & 13.046 & 13.954 \\
\hline $\mathrm{N}$ & 14.853 & 11.767 & 11.753 & $\mathrm{~N}$ & 13.002 & 14.988 & 15.201 \\
\hline $\mathrm{N}$ & 15.358 & 12.139 & 10.689 & $\mathrm{~N}$ & 13.563 & 16.090 & 15.206 \\
\hline $\mathrm{N}$ & 13.091 & 14.568 & 14.436 & $\mathrm{~N}$ & 14.627 & 11.688 & 13.171 \\
\hline $\mathrm{H}$ & 12.239 & 15.032 & 14.712 & $\mathrm{H}$ & 15.084 & 10.961 & 13.713 \\
\hline $\mathrm{H}$ & 13.178 & 13.608 & 14.735 & $\mathrm{H}$ & 13.652 & 11.869 & 13.497 \\
\hline $\mathrm{O}$ & 16.485 & 12.757 & 13.003 & $\mathrm{O}$ & 12.484 & 15.104 & 12.938 \\
\hline $\mathrm{O}$ & 16.633 & 13.798 & 9.627 & $\mathrm{O}$ & 14.804 & 17.673 & 13.926 \\
\hline $\mathrm{H}$ & 14.711 & 11.240 & 14.002 & & & & \\
\hline $\mathrm{H}$ & 16.985 & 12.984 & 9.231 & & & & \\
\hline
\end{tabular}




\begin{tabular}{|c|c|c|c|c|c|c|c|}
\hline \multicolumn{8}{|c|}{ EP- $^{-}$PBE1PBE/6-31+G*) } \\
\hline \multicolumn{4}{|c|}{$1-\mathrm{C}$} & \multicolumn{4}{|c|}{$4-\mathrm{C}$} \\
\hline $\mathrm{C}$ & 13.342 & 14.470 & 13.202 & $\mathrm{C}$ & 13.638 & 14.918 & 13.215 \\
\hline $\mathrm{C}$ & 14.148 & 13.680 & 12.363 & $\mathrm{C}$ & 14.552 & 14.097 & 12.535 \\
\hline $\mathrm{C}$ & 14.592 & 14.180 & 11.145 & $\mathrm{C}$ & 15.102 & 14.492 & 11.316 \\
\hline $\mathrm{C}$ & 14.236 & 15.442 & 10.680 & $\mathrm{C}$ & 14.765 & 15.706 & 10.730 \\
\hline $\mathrm{C}$ & 13.411 & 16.215 & 11.497 & $\mathrm{C}$ & 13.854 & 16.525 & 11.393 \\
\hline $\mathrm{C}$ & 12.975 & 15.745 & 12.736 & $\mathrm{C}$ & 13.300 & 16.141 & 12.614 \\
\hline $\mathrm{H}$ & 14.590 & 15.800 & 9.717 & $\mathrm{H}$ & 15.220 & 15.972 & 9.780 \\
\hline $\mathrm{H}$ & 13.105 & 17.209 & 11.172 & $\mathrm{H}$ & 13.567 & 17.483 & 10.963 \\
\hline $\mathrm{H}$ & 12.353 & 16.378 & 13.368 & $\mathrm{H}$ & 12.603 & 16.802 & 13.129 \\
\hline $\mathrm{C}$ & 14.541 & 12.250 & 12.644 & $\mathrm{C}$ & 15.078 & 12.757 & 12.911 \\
\hline $\mathrm{C}$ & 15.424 & 13.161 & 10.456 & $\mathrm{C}$ & 16.045 & 13.502 & 10.672 \\
\hline $\mathrm{O}$ & 16.561 & 12.814 & 11.265 & $\mathrm{O}$ & 17.115 & 13.218 & 11.873 \\
\hline $\mathrm{O}$ & 14.198 & 11.690 & 13.726 & $\mathrm{O}$ & 14.523 & 12.202 & 14.049 \\
\hline $\mathrm{N}$ & 14.264 & 11.407 & 11.376 & $\mathrm{~N}$ & 14.908 & 11.774 & 11.808 \\
\hline $\mathrm{N}$ & 14.689 & 11.876 & 10.316 & $\mathrm{~N}$ & 15.402 & 12.103 & 10.724 \\
\hline $\mathrm{N}$ & 12.987 & 14.008 & 14.456 & $\mathrm{~N}$ & 13.121 & 14.588 & 14.470 \\
\hline $\mathrm{H}$ & 12.064 & 14.273 & 14.773 & $\mathrm{H}$ & 12.179 & 14.922 & 14.625 \\
\hline $\mathrm{H}$ & 13.224 & 13.010 & 14.553 & $\mathrm{H}$ & 13.229 & 13.607 & 14.701 \\
\hline $\mathrm{O}$ & 16.108 & 12.234 & 12.511 & $\mathrm{O}$ & 16.492 & 12.798 & 13.107 \\
\hline $\mathrm{O}$ & 15.896 & 13.549 & 9.227 & $\mathrm{O}$ & 16.654 & 13.783 & 9.622 \\
\hline $\mathrm{H}$ & 16.043 & 12.721 & 8.739 & $\mathrm{H}$ & 14.587 & 11.241 & 13.909 \\
\hline
\end{tabular}




\begin{tabular}{|c|c|c|c|c|c|c|c|}
\hline \multicolumn{4}{|c|}{ EP $\left(\mathrm{M} 062 \mathrm{X} / 6-31+\mathrm{G}^{*}\right)$} & \multicolumn{4}{|c|}{$\mathbf{E P}^{2-}\left(\mathrm{M} 062 \mathrm{X} / 6-31+\mathrm{G}^{*}\right)$} \\
\hline $\mathrm{C}$ & 13.613 & 14.909 & 13.222 & $\mathrm{C}$ & 13.650 & 14.954 & 13.213 \\
\hline $\mathrm{C}$ & 14.508 & 14.102 & 12.507 & $\mathrm{C}$ & 14.572 & 14.125 & 12.555 \\
\hline $\mathrm{C}$ & 15.047 & 14.533 & 11.295 & $\mathrm{C}$ & 15.129 & 14.516 & 11.341 \\
\hline $\mathrm{C}$ & 14.729 & 15.751 & 10.723 & $\mathrm{C}$ & 14.782 & 15.720 & 10.734 \\
\hline $\mathrm{C}$ & 13.828 & 16.557 & 11.426 & $\mathrm{C}$ & 13.856 & 16.544 & 11.377 \\
\hline $\mathrm{C}$ & 13.287 & 16.151 & 12.638 & $\mathrm{C}$ & 13.301 & 16.171 & 12.606 \\
\hline $\mathrm{H}$ & 15.163 & 16.053 & 9.777 & $\mathrm{H}$ & 15.239 & 15.972 & 9.780 \\
\hline $\mathrm{H}$ & 13.542 & 17.524 & 11.021 & $\mathrm{H}$ & 13.564 & 17.495 & 10.929 \\
\hline $\mathrm{H}$ & 12.598 & 16.806 & 13.167 & $\mathrm{H}$ & 12.601 & 16.832 & 13.120 \\
\hline $\mathrm{C}$ & 15.025 & 12.741 & 12.833 & $\mathrm{C}$ & 15.001 & 12.739 & 13.005 \\
\hline $\mathrm{C}$ & 15.972 & 13.502 & 10.737 & $\mathrm{C}$ & 16.076 & 13.496 & 10.732 \\
\hline $\mathrm{O}$ & 17.032 & 13.233 & 11.693 & $\mathrm{O}$ & 17.093 & 13.212 & 11.826 \\
\hline $\mathrm{O}$ & 14.556 & 12.197 & 13.990 & $\mathrm{O}$ & 14.509 & 12.270 & 14.109 \\
\hline $\mathrm{N}$ & 14.799 & 11.794 & 11.693 & $\mathrm{~N}$ & 14.796 & 11.791 & 11.794 \\
\hline $\mathrm{N}$ & 15.283 & 12.179 & 10.628 & $\mathrm{~N}$ & 15.305 & 12.141 & 10.726 \\
\hline $\mathrm{N}$ & 13.109 & 14.565 & 14.458 & $\mathrm{~N}$ & 13.132 & 14.586 & 14.476 \\
\hline $\mathrm{H}$ & 12.256 & 15.025 & 14.741 & $\mathrm{H}$ & 12.119 & 14.656 & 14.497 \\
\hline $\mathrm{H}$ & 13.184 & 13.596 & 14.736 & $\mathrm{H}$ & 13.452 & 13.614 & 14.653 \\
\hline $\mathrm{O}$ & 16.471 & 12.767 & 12.931 & $\mathrm{O}$ & 16.492 & 12.775 & 13.074 \\
\hline $\mathrm{O}$ & 16.556 & 13.851 & 9.567 & $\mathrm{O}$ & 16.662 & 13.778 & 9.634 \\
\hline $\mathrm{H}$ & 14.757 & 11.245 & 13.981 & & & & \\
\hline $\mathrm{H}$ & 16.936 & 13.053 & 9.162 & & & & \\
\hline
\end{tabular}




\begin{tabular}{|c|c|c|c|c|c|c|c|}
\hline \multicolumn{8}{|c|}{$\mathbf{E P}^{-}\left(\mathrm{M} 062 \mathrm{X} / 6-31+\mathrm{G}^{*}\right)$} \\
\hline \multicolumn{4}{|c|}{$1-\mathrm{C}$} & \multicolumn{4}{|c|}{$4-\mathrm{C}$} \\
\hline $\mathrm{C}$ & 13.636 & 14.917 & 13.215 & $\mathrm{C}$ & 13.637 & 14.944 & 13.220 \\
\hline $\mathrm{C}$ & 14.555 & 14.101 & 12.541 & $\mathrm{C}$ & 14.542 & 14.129 & 12.521 \\
\hline $\mathrm{C}$ & 15.110 & 14.498 & 11.325 & $\mathrm{C}$ & 15.079 & 14.558 & 11.314 \\
\hline $\mathrm{C}$ & 14.775 & 15.709 & 10.735 & $\mathrm{C}$ & 14.737 & 15.770 & 10.725 \\
\hline $\mathrm{C}$ & 13.859 & 16.527 & 11.395 & $\mathrm{C}$ & 13.819 & 16.571 & 11.407 \\
\hline $\mathrm{C}$ & 13.301 & 16.141 & 12.614 & $\mathrm{C}$ & 13.284 & 16.172 & 12.631 \\
\hline $\mathrm{H}$ & 15.234 & 15.972 & 9.787 & $\mathrm{H}$ & 15.168 & 16.066 & 9.774 \\
\hline $\mathrm{H}$ & 13.573 & 17.484 & 10.965 & $\mathrm{H}$ & 13.519 & 17.528 & 10.986 \\
\hline $\mathrm{H}$ & 12.602 & 16.799 & 13.127 & $\mathrm{H}$ & 12.589 & 16.824 & 13.159 \\
\hline $\mathrm{C}$ & 15.083 & 12.755 & 12.909 & $\mathrm{C}$ & 14.957 & 12.730 & 12.936 \\
\hline $\mathrm{C}$ & 16.063 & 13.503 & 10.687 & $\mathrm{C}$ & 15.999 & 13.518 & 10.775 \\
\hline $\mathrm{O}$ & 17.111 & 13.208 & 11.843 & $\mathrm{O}$ & 17.058 & 13.263 & 11.703 \\
\hline $\mathrm{O}$ & 14.539 & 12.206 & 14.056 & $\mathrm{O}$ & 14.528 & 12.228 & 14.015 \\
\hline $\mathrm{N}$ & 14.876 & 11.775 & 11.795 & $\mathrm{~N}$ & 14.779 & 11.800 & 11.689 \\
\hline $\mathrm{N}$ & 15.360 & 12.106 & 10.713 & $\mathrm{~N}$ & 15.290 & 12.207 & 10.645 \\
\hline $\mathrm{N}$ & 13.104 & 14.587 & 14.470 & $\mathrm{~N}$ & 13.163 & 14.566 & 14.468 \\
\hline $\mathrm{H}$ & 12.154 & 14.913 & 14.601 & $\mathrm{H}$ & 12.204 & 14.827 & 14.658 \\
\hline $\mathrm{H}$ & 13.208 & 13.606 & 14.704 & $\mathrm{H}$ & 13.398 & 13.587 & 14.670 \\
\hline $\mathrm{O}$ & 16.495 & 12.797 & 13.085 & $\mathrm{O}$ & 16.507 & 12.754 & 12.940 \\
\hline $\mathrm{O}$ & 16.657 & 13.783 & 9.627 & $\mathrm{O}$ & 16.569 & 13.847 & 9.569 \\
\hline $\mathrm{H}$ & 14.641 & 11.244 & 13.953 & $\mathrm{H}$ & 16.816 & 13.003 & 9.155 \\
\hline
\end{tabular}




\begin{tabular}{|c|c|c|c|c|c|c|c|}
\hline \multicolumn{4}{|c|}{ EP (CAM-B3LYP/6-31+G*) } & \multicolumn{4}{|c|}{$\mathbf{E P}^{2-}\left(\mathrm{CAM}-\mathrm{B} 3 \mathrm{LYP} / 6-31+\mathrm{G}^{*}\right)$} \\
\hline $\mathrm{C}$ & 13.611 & 14.909 & 13.217 & $\mathrm{C}$ & 13.648 & 14.952 & 13.208 \\
\hline $\mathrm{C}$ & 14.503 & 14.099 & 12.502 & $\mathrm{C}$ & 14.558 & 14.118 & 12.542 \\
\hline $\mathrm{C}$ & 15.044 & 14.531 & 11.292 & $\mathrm{C}$ & 15.116 & 14.509 & 11.331 \\
\hline $\mathrm{C}$ & 14.727 & 15.749 & 10.725 & $\mathrm{C}$ & 14.777 & 15.718 & 10.734 \\
\hline $\mathrm{C}$ & 13.827 & 16.555 & 11.427 & $\mathrm{C}$ & 13.860 & 16.545 & 11.380 \\
\hline $\mathrm{C}$ & 13.286 & 16.150 & 12.636 & $\mathrm{C}$ & 13.305 & 16.172 & 12.607 \\
\hline $\mathrm{H}$ & 15.161 & 16.056 & 9.781 & $\mathrm{H}$ & 15.235 & 15.974 & 9.783 \\
\hline $\mathrm{H}$ & 13.543 & 17.522 & 11.021 & $\mathrm{H}$ & 13.575 & 17.499 & 10.935 \\
\hline $\mathrm{H}$ & 12.597 & 16.804 & 13.164 & $\mathrm{H}$ & 12.613 & 16.837 & 13.125 \\
\hline $\mathrm{C}$ & 15.020 & 12.741 & 12.832 & $\mathrm{C}$ & 14.988 & 12.737 & 12.998 \\
\hline $\mathrm{C}$ & 15.972 & 13.504 & 10.735 & $\mathrm{C}$ & 16.063 & 13.492 & 10.723 \\
\hline $\mathrm{O}$ & 17.038 & 13.244 & 11.697 & $\mathrm{O}$ & 17.096 & 13.232 & 11.834 \\
\hline $\mathrm{O}$ & 14.549 & 12.194 & 13.987 & $\mathrm{O}$ & 14.489 & 12.272 & 14.102 \\
\hline $\mathrm{N}$ & 14.814 & 11.793 & 11.699 & $\mathrm{~N}$ & 14.817 & 11.783 & 11.803 \\
\hline $\mathrm{N}$ & 15.301 & 12.179 & 10.632 & $\mathrm{~N}$ & 15.328 & 12.134 & 10.732 \\
\hline $\mathrm{N}$ & 13.102 & 14.560 & 14.447 & $\mathrm{~N}$ & 13.135 & 14.580 & 14.469 \\
\hline $\mathrm{H}$ & 12.268 & 15.034 & 14.758 & $\mathrm{H}$ & 12.124 & 14.662 & 14.510 \\
\hline $\mathrm{H}$ & 13.200 & 13.600 & 14.745 & $\mathrm{H}$ & 13.457 & 13.607 & 14.646 \\
\hline $\mathrm{O}$ & 16.472 & 12.773 & 12.941 & $\mathrm{O}$ & 16.489 & 12.790 & 13.090 \\
\hline $\mathrm{O}$ & 16.560 & 13.850 & 9.566 & $\mathrm{O}$ & 16.654 & 13.775 & 9.628 \\
\hline $\mathrm{H}$ & 14.730 & 11.238 & 13.968 & & & & \\
\hline $\mathrm{H}$ & 16.924 & 13.049 & 9.152 & & & & \\
\hline
\end{tabular}




\begin{tabular}{|c|c|c|c|c|c|c|c|}
\hline \multicolumn{8}{|c|}{ EP$^{-}($CAM-B3LYP/6-31+G*) } \\
\hline \multicolumn{4}{|c|}{$1-\mathrm{C}$} & \multicolumn{4}{|c|}{$4-\mathrm{C}$} \\
\hline $\mathrm{C}$ & 13.640 & 14.917 & 13.209 & $\mathrm{C}$ & 13.631 & 14.943 & 13.214 \\
\hline $\mathrm{C}$ & 14.545 & 14.094 & 12.527 & $\mathrm{C}$ & 14.526 & 14.122 & 12.511 \\
\hline $\mathrm{C}$ & 15.096 & 14.492 & 11.312 & $\mathrm{C}$ & 15.069 & 14.551 & 11.309 \\
\hline $\mathrm{C}$ & 14.764 & 15.705 & 10.729 & $\mathrm{C}$ & 14.737 & 15.769 & 10.729 \\
\hline $\mathrm{C}$ & 13.857 & 16.525 & 11.395 & $\mathrm{C}$ & 13.825 & 16.572 & 11.412 \\
\hline $\mathrm{C}$ & 13.305 & 16.140 & 12.614 & $\mathrm{C}$ & 13.286 & 16.174 & 12.632 \\
\hline $\mathrm{H}$ & 15.219 & 15.974 & 9.782 & $\mathrm{H}$ & 15.172 & 16.069 & 9.783 \\
\hline $\mathrm{H}$ & 13.574 & 17.484 & 10.966 & $\mathrm{H}$ & 13.533 & 17.533 & 10.993 \\
\hline $\mathrm{H}$ & 12.612 & 16.801 & 13.131 & $\mathrm{H}$ & 12.596 & 16.829 & 13.161 \\
\hline $\mathrm{C}$ & 15.077 & 12.752 & 12.906 & $\mathrm{C}$ & 14.937 & 12.727 & 12.928 \\
\hline $\mathrm{C}$ & 16.045 & 13.501 & 10.671 & $\mathrm{C}$ & 15.996 & 13.517 & 10.771 \\
\hline $\mathrm{O}$ & 17.117 & 13.232 & 11.876 & $\mathrm{O}$ & 17.065 & 13.280 & 11.699 \\
\hline $\mathrm{O}$ & 14.512 & 12.198 & 14.043 & $\mathrm{O}$ & 14.511 & 12.232 & 14.011 \\
\hline $\mathrm{N}$ & 14.907 & 11.771 & 11.800 & $\mathrm{~N}$ & 14.801 & 11.792 & 11.703 \\
\hline $\mathrm{N}$ & 15.399 & 12.102 & 10.717 & $\mathrm{~N}$ & 15.311 & 12.201 & 10.655 \\
\hline $\mathrm{N}$ & 13.116 & 14.585 & 14.465 & $\mathrm{~N}$ & 13.158 & 14.559 & 14.460 \\
\hline $\mathrm{H}$ & 12.178 & 14.927 & 14.626 & $\mathrm{H}$ & 12.206 & 14.827 & 14.670 \\
\hline $\mathrm{H}$ & 13.226 & 13.607 & 14.708 & $\mathrm{H}$ & 13.404 & 13.583 & 14.664 \\
\hline $\mathrm{O}$ & 16.487 & 12.806 & 13.115 & $\mathrm{O}$ & 16.515 & 12.774 & 12.947 \\
\hline $\mathrm{O}$ & 16.654 & 13.784 & 9.622 & $\mathrm{O}$ & 16.560 & 13.841 & 9.561 \\
\hline $\mathrm{H}$ & 14.604 & 11.235 & 13.932 & $\mathrm{H}$ & 16.804 & 12.996 & 9.147 \\
\hline
\end{tabular}




\begin{tabular}{|c|c|c|c|c|c|c|c|}
\hline \multicolumn{4}{|c|}{ EP $\left(\mathrm{PBE} 0 \mathrm{DH} / 6-31+\mathrm{G}^{*}\right)$} & \multicolumn{4}{|c|}{$\mathbf{E P}^{2-}\left(\mathrm{PBE} 0 \mathrm{DH} / 6-31+\mathrm{G}^{*}\right)$} \\
\hline $\mathrm{C}$ & 13.617 & 14.908 & 13.220 & $\mathrm{C}$ & 13.650 & 14.951 & 13.209 \\
\hline $\mathrm{C}$ & 14.508 & 14.100 & 12.506 & $\mathrm{C}$ & 14.562 & 14.121 & 12.546 \\
\hline $\mathrm{C}$ & 15.046 & 14.529 & 11.296 & $\mathrm{C}$ & 15.120 & 14.510 & 11.336 \\
\hline $\mathrm{C}$ & 14.728 & 15.745 & 10.727 & $\mathrm{C}$ & 14.779 & 15.715 & 10.735 \\
\hline $\mathrm{C}$ & 13.829 & 16.549 & 11.427 & $\mathrm{C}$ & 13.860 & 16.538 & 11.379 \\
\hline $\mathrm{C}$ & 13.291 & 16.145 & 12.637 & $\mathrm{C}$ & 13.305 & 16.167 & 12.606 \\
\hline $\mathrm{H}$ & 15.159 & 16.049 & 9.783 & $\mathrm{H}$ & 15.236 & 15.970 & 9.785 \\
\hline $\mathrm{H}$ & 13.543 & 17.512 & 11.023 & $\mathrm{H}$ & 13.572 & 17.489 & 10.935 \\
\hline $\mathrm{H}$ & 12.605 & 16.800 & 13.163 & $\mathrm{H}$ & 12.612 & 16.831 & 13.119 \\
\hline $\mathrm{C}$ & 15.022 & 12.746 & 12.830 & $\mathrm{C}$ & 14.987 & 12.744 & 12.996 \\
\hline $\mathrm{C}$ & 15.967 & 13.504 & 10.743 & $\mathrm{C}$ & 16.061 & 13.493 & 10.734 \\
\hline $\mathrm{O}$ & 17.024 & 13.240 & 11.699 & $\mathrm{O}$ & 17.078 & 13.227 & 11.835 \\
\hline $\mathrm{O}$ & 14.556 & 12.204 & 13.982 & $\mathrm{O}$ & 14.492 & 12.280 & 14.098 \\
\hline $\mathrm{N}$ & 14.809 & 11.806 & 11.704 & $\mathrm{~N}$ & 14.808 & 11.802 & 11.807 \\
\hline $\mathrm{N}$ & 15.295 & 12.190 & 10.638 & $\mathrm{~N}$ & 15.321 & 12.151 & 10.738 \\
\hline $\mathrm{N}$ & 13.121 & 14.567 & 14.453 & $\mathrm{~N}$ & 13.146 & 14.580 & 14.466 \\
\hline $\mathrm{H}$ & 12.263 & 15.013 & 14.731 & $\mathrm{H}$ & 12.138 & 14.650 & 14.502 \\
\hline $\mathrm{H}$ & 13.203 & 13.602 & 14.727 & $\mathrm{H}$ & 13.471 & 13.608 & 14.631 \\
\hline $\mathrm{O}$ & 16.465 & 12.773 & 12.932 & $\mathrm{O}$ & 16.475 & 12.787 & 13.081 \\
\hline $\mathrm{O}$ & 16.555 & 13.851 & 9.580 & $\mathrm{O}$ & 16.656 & 13.775 & 9.643 \\
\hline $\mathrm{H}$ & 14.734 & 11.253 & 13.957 & & & & \\
\hline $\mathrm{H}$ & 16.912 & 13.051 & 9.171 & & & & \\
\hline
\end{tabular}




\begin{tabular}{|c|c|c|c|c|c|c|c|}
\hline \multicolumn{8}{|c|}{ EP$^{-}\left(\mathrm{PBE} 0 \mathrm{DH} / 6-31+\mathrm{G}^{*}\right)$} \\
\hline \multicolumn{4}{|c|}{$1-\mathrm{C}$} & \multicolumn{4}{|c|}{$4-\mathrm{C}$} \\
\hline $\mathrm{C}$ & 13.640 & 14.915 & 13.213 & $\mathrm{C}$ & 13.637 & 14.943 & 13.214 \\
\hline $\mathrm{C}$ & 14.549 & 14.097 & 12.535 & $\mathrm{C}$ & 14.533 & 14.126 & 12.511 \\
\hline $\mathrm{C}$ & 15.101 & 14.491 & 11.322 & $\mathrm{C}$ & 15.073 & 14.555 & 11.310 \\
\hline $\mathrm{C}$ & 14.769 & 15.700 & 10.735 & $\mathrm{C}$ & 14.737 & 15.768 & 10.727 \\
\hline $\mathrm{C}$ & 13.860 & 16.518 & 11.396 & $\mathrm{C}$ & 13.823 & 16.567 & 11.409 \\
\hline $\mathrm{C}$ & 13.306 & 16.135 & 12.614 & $\mathrm{C}$ & 13.288 & 16.169 & 12.630 \\
\hline $\mathrm{H}$ & 15.224 & 15.965 & 9.789 & $\mathrm{H}$ & 15.169 & 16.069 & 9.782 \\
\hline $\mathrm{H}$ & 13.575 & 17.473 & 10.967 & $\mathrm{H}$ & 13.527 & 17.524 & 10.991 \\
\hline $\mathrm{H}$ & 12.612 & 16.796 & 13.126 & $\mathrm{H}$ & 12.598 & 16.822 & 13.156 \\
\hline $\mathrm{C}$ & 15.077 & 12.759 & 12.906 & $\mathrm{C}$ & 14.941 & 12.735 & 12.924 \\
\hline $\mathrm{C}$ & 16.046 & 13.500 & 10.688 & $\mathrm{C}$ & 15.991 & 13.520 & 10.779 \\
\hline $\mathrm{O}$ & 17.099 & 13.223 & 11.860 & $\mathrm{O}$ & 17.046 & 13.273 & 11.709 \\
\hline $\mathrm{O}$ & 14.528 & 12.209 & 14.044 & $\mathrm{O}$ & 14.511 & 12.241 & 14.006 \\
\hline $\mathrm{N}$ & 14.894 & 11.787 & 11.805 & $\mathrm{~N}$ & 14.790 & 11.810 & 11.706 \\
\hline $\mathrm{N}$ & 15.385 & 12.117 & 10.723 & $\mathrm{~N}$ & 15.301 & 12.217 & 10.659 \\
\hline $\mathrm{N}$ & 13.120 & 14.587 & 14.466 & $\mathrm{~N}$ & 13.176 & 14.561 & 14.458 \\
\hline $\mathrm{H}$ & 12.176 & 14.909 & 14.607 & $\mathrm{H}$ & 12.219 & 14.805 & 14.654 \\
\hline $\mathrm{H}$ & 13.230 & 13.610 & 14.696 & $\mathrm{H}$ & 13.426 & 13.584 & 14.646 \\
\hline $\mathrm{O}$ & 16.483 & 12.806 & 13.096 & $\mathrm{O}$ & 16.493 & 12.766 & 12.941 \\
\hline $\mathrm{O}$ & 16.652 & 13.785 & 9.636 & $\mathrm{O}$ & 16.565 & 13.841 & 9.579 \\
\hline $\mathrm{H}$ & 14.609 & 11.252 & 13.923 & $\mathrm{H}$ & 16.798 & 12.996 & 9.170 \\
\hline
\end{tabular}




\begin{tabular}{|c|c|c|c|c|c|c|c|}
\hline \multicolumn{8}{|c|}{ PI $^{-}\left(\mathrm{PBE} 1 \mathrm{PBE} / 6-31+\mathrm{G}^{*}\right)$} \\
\hline \multicolumn{4}{|c|}{$1-\mathrm{C}$} & \multicolumn{4}{|c|}{$4-\mathrm{C}$} \\
\hline $\mathrm{C}$ & -2.209 & 0.416 & 0.061 & $\mathrm{C}$ & 13.831 & 14.948 & 13.217 \\
\hline $\mathrm{C}$ & -0.811 & 0.361 & 0.321 & $\mathrm{C}$ & 14.816 & 14.186 & 12.493 \\
\hline $\mathrm{C}$ & -0.146 & -0.968 & 0.315 & $\mathrm{C}$ & 15.242 & 14.694 & 11.159 \\
\hline $\mathrm{C}$ & -0.964 & -2.116 & -0.115 & $\mathrm{C}$ & 14.515 & 15.842 & 10.596 \\
\hline $\mathrm{C}$ & -2.285 & -1.963 & -0.385 & $\mathrm{C}$ & 13.557 & 16.463 & 11.327 \\
\hline $\mathrm{C}$ & -2.947 & -0.686 & -0.263 & $\mathrm{C}$ & 13.215 & 16.050 & 12.656 \\
\hline $\mathrm{H}$ & -2.853 & -2.836 & -0.713 & $\mathrm{H}$ & 14.764 & 16.175 & 9.592 \\
\hline $\mathrm{H}$ & -4.013 & -0.611 & -0.460 & $\mathrm{H}$ & 13.030 & 17.310 & 10.889 \\
\hline $\mathrm{C}$ & -0.130 & 1.549 & 0.603 & $\mathrm{H}$ & 12.492 & 16.618 & 13.239 \\
\hline $\mathrm{C}$ & 1.101 & -1.243 & 0.700 & $\mathrm{C}$ & 15.321 & 12.993 & 13.005 \\
\hline $\mathrm{O}$ & 1.852 & 2.587 & 1.142 & $\mathrm{C}$ & 16.226 & 14.219 & 10.401 \\
\hline $\mathrm{O}$ & -0.544 & 2.730 & 0.661 & $\mathrm{O}$ & 16.713 & 11.178 & 12.722 \\
\hline $\mathrm{O}$ & 1.226 & 1.338 & 0.868 & $\mathrm{O}$ & 15.068 & 12.371 & 14.076 \\
\hline $\mathrm{O}$ & 2.211 & -1.587 & 0.938 & $\mathrm{~N}$ & 13.534 & 14.571 & 14.520 \\
\hline $\mathrm{H}$ & 1.036 & 3.162 & 1.044 & $\mathrm{H}$ & 12.598 & 14.812 & 14.816 \\
\hline $\mathrm{N}$ & -0.313 & -3.369 & -0.156 & $\mathrm{H}$ & 13.824 & 13.623 & 14.749 \\
\hline $\mathrm{H}$ & 0.592 & -3.323 & -0.619 & $\mathrm{O}$ & 16.274 & 12.414 & 12.168 \\
\hline $\mathrm{H}$ & -0.884 & -4.069 & -0.617 & $\mathrm{O}$ & 17.035 & 13.891 & 9.599 \\
\hline $\mathrm{H}$ & -2.670 & 1.399 & 0.125 & $\mathrm{H}$ & 16.170 & 11.210 & 13.563 \\
\hline
\end{tabular}




\begin{tabular}{|c|c|c|c|c|c|c|c|}
\hline \multicolumn{8}{|c|}{$\mathbf{P I}^{2-}\left(\mathrm{PBE} 1 \mathrm{PBE} / 6-31+\mathrm{G}^{*}\right)$} \\
\hline \multicolumn{4}{|c|}{$1-\mathrm{C}$} & \multicolumn{4}{|c|}{$4-\mathrm{C}$} \\
\hline $\mathrm{C}$ & -1.885 & 0.051 & -0.084 & $\mathrm{C}$ & -1.982 & -0.056 & -0.062 \\
\hline $\mathrm{C}$ & -0.536 & 0.015 & 0.271 & $\mathrm{C}$ & -0.642 & -0.081 & 0.352 \\
\hline $\mathrm{C}$ & 0.180 & -1.222 & 0.266 & $\mathrm{C}$ & 0.127 & 1.186 & 0.349 \\
\hline $\mathrm{C}$ & -0.592 & -2.412 & 0.056 & $\mathrm{C}$ & -0.604 & 2.420 & 0.034 \\
\hline $\mathrm{C}$ & -1.936 & -2.347 & -0.300 & $\mathrm{C}$ & -1.911 & 2.358 & -0.368 \\
\hline $\mathrm{C}$ & -2.588 & -1.112 & -0.403 & $\mathrm{C}$ & -2.625 & 1.133 & -0.474 \\
\hline $\mathrm{H}$ & -2.487 & -3.281 & -0.443 & $\mathrm{H}$ & -0.082 & 3.374 & 0.103 \\
\hline $\mathrm{H}$ & -3.645 & -1.068 & -0.670 & $\mathrm{H}$ & -2.432 & 3.295 & -0.591 \\
\hline $\mathrm{C}$ & 0.077 & 1.337 & 0.633 & $\mathrm{H}$ & -3.702 & 1.125 & -0.643 \\
\hline $\mathrm{C}$ & 1.648 & -1.350 & 0.013 & $\mathrm{C}$ & 0.069 & -1.320 & 0.626 \\
\hline $\mathrm{O}$ & 1.832 & 2.358 & 1.761 & $\mathrm{C}$ & 1.456 & 1.275 & 0.505 \\
\hline $\mathrm{O}$ & -0.467 & 2.408 & 0.351 & $\mathrm{O}$ & 2.221 & -2.133 & 1.171 \\
\hline $\mathrm{O}$ & 1.195 & 1.178 & 1.306 & $\mathrm{O}$ & -0.410 & -2.473 & 0.558 \\
\hline $\mathrm{O}$ & 2.371 & -2.154 & 0.621 & $\mathrm{~N}$ & -2.741 & -1.241 & -0.024 \\
\hline $\mathrm{N}$ & 0.012 & -3.695 & 0.208 & $\mathrm{H}$ & -3.341 & -1.313 & -0.839 \\
\hline $\mathrm{H}$ & 0.993 & -3.561 & 0.465 & $\mathrm{H}$ & -2.106 & -2.043 & 0.075 \\
\hline $\mathrm{H}$ & -0.015 & -4.211 & -0.670 & $\mathrm{O}$ & 1.335 & -1.046 & 0.933 \\
\hline $\mathrm{H}$ & -2.370 & 1.025 & -0.093 & $\mathrm{O}$ & 2.530 & 1.696 & 0.841 \\
\hline
\end{tabular}




\begin{tabular}{|c|c|c|c|c|c|c|c|}
\hline \multicolumn{8}{|c|}{$\mathbf{P I}^{-}\left(\mathrm{M} 062 \mathrm{X} / 6-31+\mathrm{G}^{*}\right)$} \\
\hline \multicolumn{4}{|c|}{$1-\mathrm{C}$} & \multicolumn{4}{|c|}{$4-\mathrm{C}$} \\
\hline $\mathrm{C}$ & 13.552 & 14.944 & 13.223 & $\mathrm{C}$ & 13.823 & 14.950 & 13.216 \\
\hline $\mathrm{C}$ & 14.699 & 14.127 & 12.771 & $\mathrm{C}$ & 14.814 & 14.183 & 12.497 \\
\hline $\mathrm{C}$ & 15.346 & 14.537 & 11.497 & $\mathrm{C}$ & 15.244 & 14.688 & 11.161 \\
\hline $\mathrm{C}$ & 14.925 & 15.755 & 10.886 & $\mathrm{C}$ & 14.515 & 15.839 & 10.592 \\
\hline $\mathrm{C}$ & 13.911 & 16.519 & 11.378 & $\mathrm{C}$ & 13.560 & 16.461 & 11.318 \\
\hline $\mathrm{C}$ & 13.195 & 16.065 & 12.556 & $\mathrm{C}$ & 13.213 & 16.049 & 12.654 \\
\hline $\mathrm{H}$ & 15.461 & 16.048 & 9.986 & $\mathrm{H}$ & 14.766 & 16.167 & 9.588 \\
\hline $\mathrm{H}$ & 13.611 & 17.440 & 10.889 & $\mathrm{H}$ & 13.034 & 17.307 & 10.879 \\
\hline $\mathrm{H}$ & 12.337 & 16.632 & 12.917 & $\mathrm{H}$ & 12.488 & 16.616 & 13.232 \\
\hline $\mathrm{C}$ & 15.052 & 13.123 & 13.570 & $\mathrm{C}$ & 15.321 & 12.995 & 13.014 \\
\hline $\mathrm{C}$ & 16.371 & 13.801 & 10.900 & $\mathrm{C}$ & 16.222 & 14.210 & 10.402 \\
\hline $\mathrm{O}$ & 16.696 & 12.654 & 11.636 & $\mathrm{O}$ & 16.737 & 11.192 & 12.715 \\
\hline $\mathrm{O}$ & 15.241 & 12.226 & 14.317 & $\mathrm{O}$ & 15.072 & 12.371 & 14.081 \\
\hline $\mathrm{N}$ & 12.917 & 14.516 & 14.416 & $\mathrm{~N}$ & 13.520 & 14.579 & 14.524 \\
\hline $\mathrm{H}$ & 12.046 & 15.014 & 14.569 & $\mathrm{H}$ & 12.588 & 14.838 & 14.816 \\
\hline $\mathrm{H}$ & 12.731 & 13.515 & 14.413 & $\mathrm{H}$ & 13.789 & 13.628 & 14.762 \\
\hline $\mathrm{O}$ & 17.735 & 11.939 & 10.982 & $\mathrm{O}$ & 16.278 & 12.420 & 12.171 \\
\hline $\mathrm{O}$ & 17.021 & 14.011 & 9.854 & $\mathrm{O}$ & 17.033 & 13.881 & 9.607 \\
\hline $\mathrm{H}$ & 17.869 & 12.551 & 10.208 & $\mathrm{H}$ & 16.210 & 11.195 & 13.559 \\
\hline
\end{tabular}




\begin{tabular}{|c|c|c|c|c|c|c|c|}
\hline \multicolumn{8}{|c|}{$\mathbf{P I}^{2-}\left(\mathrm{M} 062 \mathrm{X} / 6-31+\mathrm{G}^{*}\right)$} \\
\hline \multicolumn{4}{|c|}{$1-\mathrm{C}$} & \multicolumn{4}{|c|}{$4-\mathrm{C}$} \\
\hline $\mathrm{C}$ & 13.612 & 14.999 & 13.243 & $\mathrm{C}$ & 13.750 & 14.933 & 13.191 \\
\hline $\mathrm{C}$ & 14.519 & 14.079 & 12.640 & $\mathrm{C}$ & 14.662 & 14.142 & 12.462 \\
\hline $\mathrm{C}$ & 15.150 & 14.497 & 11.439 & $\mathrm{C}$ & 15.089 & 14.566 & 11.174 \\
\hline $\mathrm{C}$ & 14.812 & 15.713 & 10.841 & $\mathrm{C}$ & 14.634 & 15.816 & 10.694 \\
\hline $\mathrm{C}$ & 13.870 & 16.567 & 11.415 & $\mathrm{C}$ & 13.750 & 16.594 & 11.425 \\
\hline $\mathrm{C}$ & 13.288 & 16.208 & 12.633 & $\mathrm{C}$ & 13.289 & 16.154 & 12.667 \\
\hline $\mathrm{H}$ & 15.312 & 15.969 & 9.910 & $\mathrm{H}$ & 14.983 & 16.139 & 9.713 \\
\hline $\mathrm{H}$ & 13.621 & 17.517 & 10.943 & $\mathrm{H}$ & 13.417 & 17.561 & 11.039 \\
\hline $\mathrm{H}$ & 12.600 & 16.881 & 13.147 & $\mathrm{H}$ & 12.605 & 16.760 & 13.262 \\
\hline $\mathrm{C}$ & 14.383 & 12.621 & 13.086 & $\mathrm{C}$ & 15.141 & 12.844 & 13.070 \\
\hline $\mathrm{C}$ & 16.213 & 13.696 & 10.712 & $\mathrm{C}$ & 15.661 & 13.668 & 10.084 \\
\hline $\mathrm{O}$ & 16.845 & 12.886 & 11.517 & $\mathrm{O}$ & 16.922 & 11.383 & 13.300 \\
\hline $\mathrm{O}$ & 14.634 & 12.322 & 14.263 & $\mathrm{O}$ & 14.432 & 12.185 & 13.843 \\
\hline $\mathrm{N}$ & 12.984 & 14.697 & 14.501 & $\mathrm{~N}$ & 13.331 & 14.572 & 14.493 \\
\hline $\mathrm{H}$ & 12.002 & 14.466 & 14.344 & $\mathrm{H}$ & 12.374 & 14.863 & 14.662 \\
\hline $\mathrm{H}$ & 13.425 & 13.858 & 14.878 & $\mathrm{H}$ & 13.448 & 13.565 & 14.623 \\
\hline $\mathrm{O}$ & 17.930 & 12.156 & 10.941 & $\mathrm{O}$ & 16.358 & 12.556 & 12.713 \\
\hline $\mathrm{O}$ & 16.439 & 13.887 & 9.518 & $\mathrm{O}$ & 16.654 & 14.039 & 9.452 \\
\hline
\end{tabular}




\begin{tabular}{|c|c|c|c|c|c|c|c|}
\hline \multicolumn{8}{|c|}{ PI $^{-}($CAM-B3LYP/6-31+G*) } \\
\hline \multicolumn{4}{|c|}{$1-\mathrm{C}$} & \multicolumn{4}{|c|}{$4-\mathrm{C}$} \\
\hline $\mathrm{C}$ & 13.550 & 14.943 & 13.223 & $\mathrm{C}$ & 13.743 & 14.896 & 13.161 \\
\hline $\mathrm{C}$ & 14.692 & 14.120 & 12.773 & $\mathrm{C}$ & 14.809 & 14.181 & 12.497 \\
\hline $\mathrm{C}$ & 15.350 & 14.533 & 11.503 & $\mathrm{C}$ & 15.331 & 14.748 & 11.219 \\
\hline $\mathrm{C}$ & 14.935 & 15.756 & 10.896 & $\mathrm{C}$ & 14.625 & 15.910 & 10.645 \\
\hline $\mathrm{C}$ & 13.923 & 16.519 & 11.386 & $\mathrm{C}$ & 13.600 & 16.480 & 11.315 \\
\hline $\mathrm{C}$ & 13.202 & 16.067 & 12.560 & $\mathrm{C}$ & 13.154 & 16.002 & 12.595 \\
\hline $\mathrm{H}$ & 15.473 & 16.053 & 10.000 & $\mathrm{H}$ & 14.950 & 16.290 & 9.682 \\
\hline $\mathrm{H}$ & 13.628 & 17.444 & 10.899 & $\mathrm{H}$ & 13.094 & 17.336 & 10.872 \\
\hline $\mathrm{H}$ & 12.347 & 16.640 & 12.917 & $\mathrm{H}$ & 12.369 & 16.529 & 13.132 \\
\hline $\mathrm{C}$ & 15.041 & 13.110 & 13.562 & $\mathrm{C}$ & 15.303 & 12.989 & 13.009 \\
\hline $\mathrm{C}$ & 16.371 & 13.798 & 10.906 & $\mathrm{C}$ & 16.371 & 14.317 & 10.521 \\
\hline $\mathrm{O}$ & 16.695 & 12.640 & 11.628 & $\mathrm{O}$ & 16.774 & 11.215 & 12.786 \\
\hline $\mathrm{O}$ & 15.222 & 12.204 & 14.304 & $\mathrm{O}$ & 14.982 & 12.321 & 14.033 \\
\hline $\mathrm{N}$ & 12.902 & 14.521 & 14.410 & $\mathrm{~N}$ & 13.342 & 14.458 & 14.418 \\
\hline $\mathrm{H}$ & 12.039 & 15.028 & 14.570 & $\mathrm{H}$ & 12.389 & 14.688 & 14.661 \\
\hline $\mathrm{H}$ & 12.714 & 13.521 & 14.426 & $\mathrm{H}$ & 13.636 & 13.513 & 14.651 \\
\hline $\mathrm{O}$ & 17.741 & 11.935 & 10.954 & $\mathrm{O}$ & 16.341 & 12.460 & 12.234 \\
\hline $\mathrm{O}$ & 17.028 & 14.013 & 9.861 & $\mathrm{O}$ & 17.249 & 14.039 & 9.775 \\
\hline $\mathrm{H}$ & 17.861 & 12.572 & 10.191 & $\mathrm{H}$ & 16.166 & 11.197 & 13.580 \\
\hline
\end{tabular}




\begin{tabular}{|c|c|c|c|c|c|c|c|}
\hline \multicolumn{8}{|c|}{ PI $^{2-}\left(\mathrm{CAM}-\mathrm{B} 3 \mathrm{LYP} / 6-31+\mathrm{G}^{*}\right)$} \\
\hline \multicolumn{4}{|c|}{$1-\mathrm{C}$} & \multicolumn{4}{|c|}{$4-\mathrm{C}$} \\
\hline $\mathrm{C}$ & 13.614 & 14.959 & 13.258 & $\mathrm{C}$ & 13.755 & 14.935 & 13.187 \\
\hline $\mathrm{C}$ & 14.476 & 14.017 & 12.634 & $\mathrm{C}$ & 14.661 & 14.139 & 12.455 \\
\hline $\mathrm{C}$ & 15.128 & 14.425 & 11.451 & $\mathrm{C}$ & 15.073 & 14.561 & 11.162 \\
\hline $\mathrm{C}$ & 14.859 & 15.667 & 10.871 & $\mathrm{C}$ & 14.623 & 15.814 & 10.688 \\
\hline $\mathrm{C}$ & 13.935 & 16.535 & 11.450 & $\mathrm{C}$ & 13.749 & 16.594 & 11.425 \\
\hline $\mathrm{C}$ & 13.321 & 16.183 & 12.647 & $\mathrm{C}$ & 13.295 & 16.156 & 12.668 \\
\hline $\mathrm{H}$ & 15.366 & 15.930 & 9.948 & $\mathrm{H}$ & 14.967 & 16.137 & 9.707 \\
\hline $\mathrm{H}$ & 13.713 & 17.494 & 10.983 & $\mathrm{H}$ & 13.419 & 17.562 & 11.040 \\
\hline $\mathrm{H}$ & 12.638 & 16.873 & 13.147 & $\mathrm{H}$ & 12.617 & 16.764 & 13.266 \\
\hline $\mathrm{C}$ & 14.393 & 12.504 & 12.994 & $\mathrm{C}$ & 15.138 & 12.848 & 13.069 \\
\hline $\mathrm{C}$ & 16.158 & 13.598 & 10.702 & $\mathrm{C}$ & 15.651 & 13.674 & 10.080 \\
\hline $\mathrm{O}$ & 17.142 & 13.247 & 11.492 & $\mathrm{O}$ & 16.931 & 11.384 & 13.330 \\
\hline $\mathrm{O}$ & 14.152 & 12.254 & 14.201 & $\mathrm{O}$ & 14.425 & 12.190 & 13.843 \\
\hline $\mathrm{N}$ & 13.048 & 14.682 & 14.526 & $\mathrm{~N}$ & 13.337 & 14.566 & 14.484 \\
\hline $\mathrm{H}$ & 12.045 & 14.849 & 14.543 & $\mathrm{H}$ & 12.386 & 14.862 & 14.672 \\
\hline $\mathrm{H}$ & 13.278 & 13.712 & 14.780 & $\mathrm{H}$ & 13.460 & 13.558 & 14.611 \\
\hline $\mathrm{O}$ & 18.288 & 12.643 & 10.861 & $\mathrm{O}$ & 16.361 & 12.558 & 12.727 \\
\hline $\mathrm{O}$ & 16.083 & 13.446 & 9.485 & $\mathrm{O}$ & 16.653 & 14.038 & 9.453 \\
\hline
\end{tabular}




\begin{tabular}{|c|c|c|c|c|c|c|c|}
\hline \multicolumn{8}{|c|}{ PI $^{-}\left(\mathrm{PBE} 0 \mathrm{DH} / 6-31+\mathrm{G}^{*}\right)$} \\
\hline \multicolumn{4}{|c|}{$1-\mathrm{C}$} & \multicolumn{4}{|c|}{$4-\mathrm{C}$} \\
\hline $\mathrm{C}$ & 13.550 & 14.933 & 13.215 & $\mathrm{C}$ & 13.844 & 14.958 & 13.222 \\
\hline $\mathrm{C}$ & 14.714 & 14.145 & 12.788 & $\mathrm{C}$ & 14.817 & 14.188 & 12.494 \\
\hline $\mathrm{C}$ & 15.346 & 14.535 & 11.506 & $\mathrm{C}$ & 15.251 & 14.701 & 11.171 \\
\hline $\mathrm{C}$ & 14.945 & 15.759 & 10.907 & $\mathrm{C}$ & 14.494 & 15.815 & 10.588 \\
\hline $\mathrm{C}$ & 13.930 & 16.522 & 11.397 & $\mathrm{C}$ & 13.541 & 16.436 & 11.316 \\
\hline $\mathrm{C}$ & 13.196 & 16.056 & 12.549 & $\mathrm{C}$ & 13.226 & 16.050 & 12.659 \\
\hline $\mathrm{H}$ & 15.484 & 16.056 & 10.014 & $\mathrm{H}$ & 14.719 & 16.125 & 9.574 \\
\hline $\mathrm{H}$ & 13.642 & 17.448 & 10.915 & $\mathrm{H}$ & 12.991 & 17.258 & 10.866 \\
\hline $\mathrm{H}$ & 12.321 & 16.607 & 12.887 & $\mathrm{H}$ & 12.513 & 16.624 & 13.241 \\
\hline $\mathrm{C}$ & 15.122 & 13.197 & 13.627 & $\mathrm{C}$ & 15.304 & 12.988 & 12.995 \\
\hline $\mathrm{C}$ & 16.348 & 13.783 & 10.899 & $\mathrm{C}$ & 16.272 & 14.266 & 10.446 \\
\hline $\mathrm{O}$ & 16.669 & 12.639 & 11.625 & $\mathrm{O}$ & 16.678 & 11.172 & 12.696 \\
\hline $\mathrm{O}$ & 15.334 & 12.310 & 14.380 & $\mathrm{O}$ & 15.049 & 12.365 & 14.060 \\
\hline $\mathrm{N}$ & 12.897 & 14.492 & 14.383 & $\mathrm{~N}$ & 13.561 & 14.592 & 14.529 \\
\hline $\mathrm{H}$ & 12.030 & 14.989 & 14.533 & $\mathrm{H}$ & 12.629 & 14.830 & 14.825 \\
\hline $\mathrm{H}$ & 12.709 & 13.496 & 14.365 & $\mathrm{H}$ & 13.843 & 13.645 & 14.755 \\
\hline $\mathrm{O}$ & 17.686 & 11.920 & 10.949 & $\mathrm{O}$ & 16.245 & 12.407 & 12.153 \\
\hline $\mathrm{O}$ & 16.988 & 13.985 & 9.845 & $\mathrm{O}$ & 17.101 & 13.949 & 9.664 \\
\hline $\mathrm{H}$ & 17.803 & 12.545 & 10.183 & $\mathrm{H}$ & 16.146 & 11.197 & 13.535 \\
\hline
\end{tabular}




\begin{tabular}{|c|c|c|c|c|c|c|c|}
\hline \multicolumn{8}{|c|}{$\mathbf{P I}^{2-}\left(\mathrm{PBE} 0 \mathrm{DH} / 6-31+\mathrm{G}^{*}\right)$} \\
\hline \multicolumn{4}{|c|}{$1-\mathrm{C}$} & \multicolumn{4}{|c|}{$4-\mathrm{C}$} \\
\hline $\mathrm{C}$ & 13.631 & 14.968 & 13.250 & $\mathrm{C}$ & 13.719 & 14.952 & 13.197 \\
\hline $\mathrm{C}$ & 14.508 & 14.041 & 12.622 & $\mathrm{C}$ & 14.701 & 14.189 & 12.541 \\
\hline $\mathrm{C}$ & 15.145 & 14.468 & 11.432 & $\mathrm{C}$ & 15.134 & 14.594 & 11.224 \\
\hline $\mathrm{C}$ & 14.823 & 15.690 & 10.847 & $\mathrm{C}$ & 14.656 & 15.844 & 10.715 \\
\hline $\mathrm{C}$ & 13.874 & 16.531 & 11.422 & $\mathrm{C}$ & 13.707 & 16.570 & 11.395 \\
\hline $\mathrm{C}$ & 13.292 & 16.172 & 12.633 & $\mathrm{C}$ & 13.193 & 16.121 & 12.620 \\
\hline $\mathrm{H}$ & 15.323 & 15.956 & 9.923 & $\mathrm{H}$ & 15.032 & 16.184 & 9.754 \\
\hline $\mathrm{H}$ & 13.619 & 17.478 & 10.952 & $\mathrm{H}$ & 13.370 & 17.524 & 10.990 \\
\hline $\mathrm{H}$ & 12.606 & 16.849 & 13.141 & $\mathrm{H}$ & 12.489 & 16.722 & 13.190 \\
\hline $\mathrm{C}$ & 14.351 & 12.560 & 12.971 & $\mathrm{C}$ & 15.208 & 12.935 & 13.142 \\
\hline $\mathrm{C}$ & 16.205 & 13.678 & 10.711 & $\mathrm{C}$ & 15.717 & 13.755 & 10.231 \\
\hline $\mathrm{O}$ & 16.985 & 13.058 & 11.554 & $\mathrm{O}$ & 16.766 & 11.227 & 12.919 \\
\hline $\mathrm{O}$ & 14.278 & 12.248 & 14.176 & $\mathrm{O}$ & 14.714 & 12.406 & 14.153 \\
\hline $\mathrm{N}$ & 13.095 & 14.689 & 14.530 & $\mathrm{~N}$ & 13.292 & 14.616 & 14.491 \\
\hline $\mathrm{H}$ & 12.083 & 14.748 & 14.524 & $\mathrm{H}$ & 12.302 & 14.780 & 14.605 \\
\hline $\mathrm{H}$ & 13.385 & 13.745 & 14.797 & $\mathrm{H}$ & 13.564 & 13.652 & 14.695 \\
\hline $\mathrm{O}$ & 18.114 & 12.399 & 10.997 & $\mathrm{O}$ & 16.211 & 12.452 & 12.456 \\
\hline $\mathrm{O}$ & 16.323 & 13.740 & 9.490 & $\mathrm{O}$ & 16.725 & 13.818 & 9.549 \\
\hline
\end{tabular}


Absolute free energies of $S_{0}$ state in gas phase and solvation energies of the optimized stationary points at PBE1PBE/6-31+G*, M062X/6-31+G*, CAM-B3LYP/6-31+G* and PBE0DH/6-31+G* levels, respectively (unit: Hartree). LH2: luminol, LH': luminol monoanion, $\mathbf{L}^{2-:}$ luminol dianion

\begin{tabular}{|c|c|c|c|c|c|c|c|}
\hline \multicolumn{8}{|c|}{ PBE1PBE/6-31+G* } \\
\hline & \multirow[b]{2}{*}{$G_{\text {gas }}\left(\mathrm{S}_{0}\right)$} & \multicolumn{2}{|c|}{ UAHF } & \multicolumn{2}{|c|}{ UAKS } & \multicolumn{2}{|c|}{ UFF } \\
\hline & & $\Delta G_{\text {pol.solu.-solv. }}$ & $\Delta G_{\text {solv }}$ & $\Delta G_{\text {pol.solu.-solv. }}$ & $\Delta G_{\text {solv }}$ & $\Delta G_{\text {pol.solu.-solv. }}$ & $\Delta G_{\text {solv }}$ \\
\hline $\mathbf{L H}_{2}$ & -622.99223800 & -0.06599739 & -0.04801593 & -0.07166810 & -0.05246215 & -0.02015328 & -0.01134661 \\
\hline $\mathbf{L}^{2-}$ & -621.76675100 & -0.36129339 & -0.32334661 & -0.37347135 & -0.33150597 & -0.31829737 & -0.29525099 \\
\hline 1-C $\mathbf{L} \mathbf{H}^{-}$ & -622.46570500 & -0.12243293 & -0.10315537 & -0.13343719 & -0.11164940 & -0.09918143 & -0.08627888 \\
\hline 4-C $\mathbf{L H}^{-}$ & -622.46867700 & -0.11830933 & -0.10044621 & -0.12880305 & -0.10858964 & -0.09575709 & -0.08400000 \\
\hline EP & -773.12343100 & -0.05489581 & -0.03987250 & -0.05847703 & -0.04288446 & -0.01631052 & -0.00710756 \\
\hline $\mathbf{E P}^{2-}$ & -771.93822500 & -0.34785598 & -0.31606374 & -0.35208076 & -0.31923505 & -0.30833082 & -0.28776095 \\
\hline $1-\mathrm{C} \mathbf{E P}^{-}$ & -772.59611600 & -0.14440498 & -0.11708366 & -0.14874294 & -0.12041434 & -0.10561058 & -0.08882868 \\
\hline 4-C EP- & -772.60440300 & -0.13415234 & -0.11040637 & -0.13763875 & -0.11321115 & -0.09871789 & -0.08412749 \\
\hline 1-C LHOOH & -773.13073700 & -0.06228740 & -0.04341035 & -0.06699885 & -0.04723505 & -0.02114276 & -0.00898804 \\
\hline 4-C LHOOH & -773.12358100 & -0.06183249 & -0.04239043 & -0.06629366 & -0.04596015 & -0.02096283 & -0.00830278 \\
\hline 1-C L-OOH & -772.61037700 & -0.13082857 & -0.10755378 & -0.13646961 & -0.11188844 & -0.10050985 & -0.08412749 \\
\hline 4-C L-OOH & -772.61236800 & -0.12000507 & -0.09996812 & -0.12448445 & -0.10353784 & -0.09325847 & -0.07883665 \\
\hline 1-C LHOO' & -772.58096300 & -0.14124217 & -0.11351394 & -0.14923951 & -0.11920318 & -0.10580736 & -0.08755378 \\
\hline 4-C LHOO' & -772.57758200 & -0.14009627 & -0.11332270 & -0.14963144 & -0.12063745 & -0.10406992 & -0.08623107 \\
\hline 1-C PI' & -663.22238700 & -0.10619722 & -0.08956175 & -0.11439623 & -0.09598406 & -0.08488934 & -0.07284462 \\
\hline 4-C PI' & -663.22902500 & -0.09676246 & -0.08333067 & -0.10262475 & -0.08820717 & -0.07971050 & -0.06970517 \\
\hline 1-C PI ${ }^{2-}$ & -662.53247200 & -0.34860262 & -0.30962549 & -0.36089808 & -0.31901195 & -0.31294567 & -0.28540239 \\
\hline 4-C PI ${ }^{2-}$ & -662.53529300 & -0.34090573 & -0.30509960 & -0.35256369 & -0.31340239 & -0.30768189 & -0.28293227 \\
\hline
\end{tabular}


PBE1PBE/6-31+G*

\begin{tabular}{|c|c|c|c|c|c|c|}
\hline & \multicolumn{2}{|c|}{ UA0 } & \multicolumn{2}{|c|}{ Bondi } & \multicolumn{2}{|c|}{ Pauling } \\
\hline & $\Delta G_{\text {pol.solu.-solv }}$ & $\Delta G_{\text {solv }}$ & $\Delta G_{\text {pol.solu.-solv }}$ & $\Delta G_{\text {solv. }}$ & $\Delta G_{\text {pol.solu.-solv }}$ & $\Delta G_{\text {solv }}$ \\
\hline $\mathbf{L H}_{2}$ & -0.02601045 & -0.01628685 & -0.03509651 & -0.02312350 & -0.04046363 & -0.02717131 \\
\hline $\mathbf{L}^{2-}$ & -0.31883587 & -0.29456573 & -0.35104386 & -0.31819920 & -0.36025886 & -0.32417529 \\
\hline 1-C $\mathbf{L} \mathbf{H}^{-}$ & -0.10154476 & -0.08792031 & -0.11744999 & -0.09985657 & -0.12355778 & -0.10404780 \\
\hline 4-C $\mathbf{L H}^{-}$ & -0.09803285 & -0.08556175 & -0.11314402 & -0.09701992 & -0.11862524 & -0.10086055 \\
\hline EP & -0.02430274 & -0.01416733 & -0.03209184 & -0.01797609 & -0.03209184 & -0.02082868 \\
\hline $\mathbf{E P}^{2-}$ & -0.30862029 & -0.28718725 & -0.34487963 & -0.30820717 & -0.34487963 & -0.31434262 \\
\hline 1-C EP' & -0.10935302 & -0.09145816 & -0.13023892 & -0.10210358 & -0.13023892 & -0.10584860 \\
\hline 4-C EP- & -0.10214250 & -0.08651792 & -0.12034448 & -0.09627091 & -0.12034448 & -0.09953784 \\
\hline 1-C LHOOH & -0.02839164 & -0.01521912 & -0.03573573 & -0.02094023 & -0.04124479 & -0.02572111 \\
\hline 4-C LHOOH & -0.02831469 & -0.01456573 & -0.03524248 & -0.02004780 & -0.04087333 & -0.02490836 \\
\hline 1-C L-OOH & -0.10134541 & -0.08452589 & -0.11847444 & -0.09735458 & -0.12541694 & -0.10258167 \\
\hline 4-C L-OOH & -0.09373177 & -0.07898007 & -0.10886295 & -0.09067729 & -0.11518826 & -0.09563346 \\
\hline 1-C LHOO' & -0.10935042 & -0.09013545 & -0.12723827 & -0.10264541 & -0.13517634 & -0.10804780 \\
\hline 4-C LHOO' & -0.10740623 & -0.08871713 & -0.12590351 & -0.10188047 & -0.13503277 & -0.10815936 \\
\hline 1-C PI' & -0.08517056 & -0.07271713 & -0.09704942 & -0.08226294 & -0.10018987 & -0.08457370 \\
\hline 4-C PI' & -0.07919027 & -0.06892430 & -0.08937304 & -0.07741832 & -0.09181265 & -0.07933067 \\
\hline $1-\mathrm{C} \mathbf{P I}^{2-}$ & -0.31388951 & -0.28495617 & -0.34229535 & -0.30608764 & -0.35562801 & -0.31370517 \\
\hline 4-C PI ${ }^{2-}$ & -0.30817464 & -0.28191235 & -0.33486696 & -0.30213545 & -0.34566928 & -0.30847808 \\
\hline
\end{tabular}




\begin{tabular}{|c|c|c|c|c|c|c|c|}
\hline \multicolumn{8}{|c|}{ M062X/6-31+G* } \\
\hline & \multicolumn{3}{|c|}{ UAHF } & \multicolumn{2}{|c|}{ UAKS } & \multicolumn{2}{|c|}{ UFF } \\
\hline & $G_{\text {gas }}\left(\mathrm{S}_{0}\right)$ & $\Delta G_{\text {pol.solu.-solv. }}$ & $\Delta G_{\text {solv }}$ & $\Delta G_{\text {pol.solu.-solv. }}$ & $\Delta G_{\text {solv }}$ & $\Delta G_{\text {pol.solu.-solv. }}$ & $\Delta G_{\text {solv }}$ \\
\hline $\mathbf{L H}_{2}$ & -623.42994100 & -0.06514681 & -0.04753784 & -0.07098222 & -0.05211155 & -0.01948569 & -0.01080478 \\
\hline $\mathbf{L}^{2-}$ & -622.20904000 & -0.36337652 & -0.32615139 & -0.37580239 & -0.33472509 & -0.31997547 & -0.29727490 \\
\hline 1-C $\mathbf{L} \mathbf{H}^{-}$ & -622.90565700 & -0.12363651 & -0.10438247 & -0.13497693 & -0.11314741 & -0.09999610 & -0.08709163 \\
\hline 4-C $\mathbf{L H}^{-}$ & -622.90842200 & -0.11978814 & -0.10176892 & -0.13065587 & -0.11021513 & -0.09676691 & -0.08489243 \\
\hline EP & -773.66620700 & -0.05617683 & -0.04111553 & -0.05980940 & -0.04415936 & -0.05617683 & -0.04111553 \\
\hline $\mathbf{E P}^{2-}$ & -772.48085100 & -0.34995156 & -0.31880478 & -0.35445805 & -0.32218326 & -0.34995156 & -0.31880478 \\
\hline 1-C EP- & -773.13853700 & -0.14624901 & -0.11912350 & -0.15077503 & -0.12261354 & -0.14624901 & -0.11912350 \\
\hline 4-C EP- & -773.14662700 & -0.13638322 & -0.11250996 & -0.14001286 & -0.11545816 & -0.13638322 & -0.11250996 \\
\hline 1-C LHOOH & -773.67336600 & -0.06207280 & -0.04368127 & -0.06689430 & -0.04768127 & -0.02040716 & -0.00868525 \\
\hline 4-C LHOOH & -773.66759400 & -0.06187466 & -0.04275697 & -0.06630515 & -0.04635856 & -0.02049991 & -0.00812749 \\
\hline 1-C L-OOH & -773.15006800 & -0.13122498 & -0.10825498 & -0.13701505 & -0.11276494 & -0.10029239 & -0.08401593 \\
\hline 4-C L-OOH & -773.15462700 & -0.12142006 & -0.10094023 & -0.12584621 & -0.10449402 & -0.09331625 & -0.07853386 \\
\hline 1-C LHOO- & -773.11970800 & -0.15240981 & -0.12529083 & -0.16250288 & -0.13305179 & -0.11321668 & -0.09391235 \\
\hline 4-C LHOO- & -773.13004900 & -0.13356570 & -0.11152191 & -0.14018043 & -0.11643027 & -0.09927511 & -0.08371314 \\
\hline 1-C $\mathbf{P I}^{-}$ & -663.68667600 & -0.10745963 & -0.09072509 & -0.11583432 & -0.09727490 & -0.08518047 & -0.07314741 \\
\hline 4-C PI' & -663.69298000 & -0.09827819 & -0.08460557 & -0.10453635 & -0.08983266 & -0.08029212 & -0.07018326 \\
\hline 1-C $\mathbf{P I}^{2-}$ & -662.99782500 & -0.34968228 & -0.31294023 & -0.36239379 & -0.32298007 & -0.31428841 & -0.28784063 \\
\hline 4-C PI $\mathbf{P I}^{2-}$ & -662.99804100 & -0.35787100 & -0.31639840 & -0.37032833 & -0.32562549 & -0.32048957 & -0.29115537 \\
\hline
\end{tabular}




\begin{tabular}{|c|c|c|c|c|c|c|}
\hline \multicolumn{7}{|c|}{ M062X/6-31+G* } \\
\hline & \multicolumn{2}{|c|}{ UA0 } & \multicolumn{2}{|c|}{ Bondi } & \multicolumn{2}{|c|}{ Pauling } \\
\hline & $\Delta G_{\text {pol.solu.-solv. }}$ & $\Delta G_{\text {solv }}$ & $\Delta G_{\text {pol.solu.-solv. }}$ & $\Delta G_{\text {solv. }}$ & $\Delta G_{\text {pol.solu.-solv. }}$ & $\Delta G_{\text {solv }}$ \\
\hline $\mathbf{L H}_{2}$ & -0.02531276 & -0.01572908 & -0.03418483 & -0.02248605 & -0.03972723 & -0.02674103 \\
\hline $\mathbf{L}^{2-}$ & -0.32044121 & -0.29660557 & -0.35322937 & -0.32097211 & -0.36280966 & -0.32739442 \\
\hline 1-C $\mathbf{L H}^{-}$ & -0.10235834 & -0.08874900 & -0.11865566 & -0.10108366 & -0.12511767 & -0.10560956 \\
\hline 4-C LH' & -0.09903459 & -0.08648605 & -0.11466977 & -0.09839043 & -0.12050902 & -0.10253386 \\
\hline EP & -0.02489320 & -0.01474103 & -0.02979881 & -0.01870916 & -0.03304082 & -0.02180079 \\
\hline $\mathbf{E P}^{2-}$ & -0.30994091 & -0.28884462 & -0.33771586 & -0.31061354 & -0.34725335 & -0.31724302 \\
\hline $1-\mathrm{C} \mathbf{E P}-$ & -0.11043606 & -0.09263745 & -0.12588097 & -0.10369721 & -0.13201487 & -0.10779282 \\
\hline 4-C EP- & -0.10346526 & -0.08772908 & -0.11734158 & -0.09794422 & -0.12254896 & -0.10156175 \\
\hline 1-C LHOOH & -0.02792017 & -0.01515537 & -0.03469954 & -0.02057370 & -0.04002148 & -0.02530677 \\
\hline 4-C LHOOH & -0.02797225 & -0.01450199 & -0.03473274 & -0.01995219 & -0.04050141 & -0.02498804 \\
\hline 1-C L-OOH & -0.10127500 & -0.08457370 & -0.11791760 & -0.09717928 & -0.12489426 & -0.10256573 \\
\hline 4-C L-OOH & -0.09397351 & -0.07885258 & -0.10886743 & -0.09039043 & -0.11504980 & -0.09536254 \\
\hline 1-C LHOO' & -0.11680314 & -0.09663745 & -0.13789497 & -0.11206374 & -0.14853990 & -0.11982470 \\
\hline 4-C LHOO' & -0.10225533 & -0.08602390 & -0.11870114 & -0.09816733 & -0.12622989 & -0.10369721 \\
\hline $1-\mathrm{C} \mathbf{P I}^{-}$ & -0.08554534 & -0.07311553 & -0.09761916 & -0.08280478 & -0.10090375 & -0.08525896 \\
\hline 4-C PI' & -0.07985465 & -0.06949800 & -0.09032010 & -0.07818326 & -0.09290665 & -0.08022310 \\
\hline $1-\mathrm{C} \mathbf{P I}^{2-}$ & -0.31505910 & -0.28742629 & -0.34367436 & -0.30932270 & -0.35703384 & -0.31762549 \\
\hline 4-C $\mathbf{P I}^{2-}$ & -0.32152751 & -0.29086852 & -0.35125369 & -0.31263745 & -0.36455862 & -0.32055776 \\
\hline
\end{tabular}




\begin{tabular}{|c|c|c|c|c|c|c|c|}
\hline \multicolumn{8}{|c|}{ CAM-B3LYP/6-31+G* } \\
\hline & \multirow[b]{2}{*}{$G_{\text {gas }}\left(\mathrm{S}_{0}\right)$} & \multicolumn{2}{|c|}{ UAHF } & \multicolumn{2}{|c|}{ UAKS } & \multicolumn{2}{|c|}{ UFF } \\
\hline & & $\Delta G_{\text {pol.solu.-solv. }}$ & $\Delta G_{\text {solv }}$ & $\Delta G_{\text {pol.solu.-solv. }}$ & $\Delta G_{\text {solv }}$ & $\Delta G_{\text {pol.solu.-solv. }}$ & $\Delta G_{\text {solv. }}$ \\
\hline $\mathbf{L H}_{2}$ & -623.39513600 & -0.06559349 & -0.04790438 & -0.07121159 & -0.05231872 & -0.02007239 & -0.01131474 \\
\hline $\mathbf{L}^{2-}$ & -622.17220900 & -0.36133324 & -0.32433466 & -0.37327632 & -0.33243027 & -0.31946091 & -0.29657370 \\
\hline 1-C $\mathbf{L H}^{-}$ & -622.86962700 & -0.12254346 & -0.10350597 & -0.13353041 & -0.11196812 & -0.09982559 & -0.08690039 \\
\hline 4-C LH' & -622.87228800 & -0.11848734 & -0.10071713 & -0.12903548 & -0.10887649 & -0.09640977 & -0.08454183 \\
\hline EP & -773.63466900 & -0.05533922 & -0.04043027 & -0.05884063 & -0.04339442 & -0.01622915 & -0.00707569 \\
\hline $\mathbf{E P}^{2-}$ & -772.45524200 & -0.34698915 & -0.31595219 & -0.35125919 & -0.31915537 & -0.30850902 & -0.28807968 \\
\hline 1-C EP- & -773.11008700 & -0.14437132 & -0.11749800 & -0.14866344 & -0.12081274 & -0.10581437 & -0.08908366 \\
\hline 4-C EP- & -773.11795400 & -0.13471271 & -0.11109163 & -0.13821551 & -0.11391235 & -0.09925179 & -0.08452589 \\
\hline 1-C LHOOH & -773.64434900 & -0.06253145 & -0.04395219 & -0.06718537 & -0.04780876 & -0.02083812 & -0.00884462 \\
\hline 4-C LHOOH & -773.63775200 & -0.06221523 & -0.04310756 & -0.06661132 & -0.04667729 & -0.02074954 & -0.00828685 \\
\hline 1-C L-OOH & -773.12602100 & -0.13030488 & -0.10780876 & -0.13586477 & -0.11214342 & -0.10011606 & -0.08398406 \\
\hline 4-C L-OOH & -773.12842200 & -0.11996655 & -0.10007968 & -0.12435906 & -0.10360159 & -0.09316979 & -0.07861354 \\
\hline 1-C LHOO- & -773.09536500 & -0.14957021 & -0.12151394 & -0.15950020 & -0.12929083 & -0.11193294 & -0.09247808 \\
\hline 4-C LHOO' & -773.09537200 & -0.14400020 & -0.11821513 & -0.15362850 & -0.12568924 & -0.10694651 & -0.08882868 \\
\hline 1-C PI' & -663.66245100 & -0.10559805 & -0.08914741 & -0.11387725 & -0.09564940 & -0.08434596 & -0.07233466 \\
\hline 4-C PI' & -663.66966100 & -0.09603254 & -0.08278884 & -0.10221652 & -0.08795219 & -0.07900034 & -0.06906772 \\
\hline $1-\mathrm{C} \mathbf{P I}^{2-}$ & -662.97968300 & -0.35123040 & -0.31292430 & -0.36235458 & -0.32135458 & -0.31712094 & -0.28929083 \\
\hline 4-C PI ${ }^{2-}$ & -662.98046800 & -0.35509016 & -0.31388047 & -0.36715115 & -0.32270916 & -0.31909577 & -0.28968924 \\
\hline
\end{tabular}




\begin{tabular}{|c|c|c|c|c|c|c|}
\hline \multicolumn{7}{|c|}{ CAM-B3LYP/6-31+G* } \\
\hline & \multicolumn{2}{|c|}{ UA0 } & \multicolumn{2}{|c|}{ Bondi } & \multicolumn{2}{|c|}{ Pauling } \\
\hline & $\Delta G_{\text {pol.solu.-solv. }}$ & $\Delta G_{\text {solv }}$ & $\Delta G_{\text {pol.solu.-solv. }}$ & $\Delta G_{\text {solv. }}$ & $\Delta G_{\text {pol.solu.-solv. }}$ & $\Delta G_{\text {solv }}$ \\
\hline $\mathbf{L H}_{2}$ & -0.02581383 & -0.01617529 & -0.03505828 & -0.02320318 & -0.04114867 & -0.02782470 \\
\hline $\mathbf{L}^{2-}$ & -0.31995913 & -0.29601593 & -0.35164654 & -0.31929880 & -0.36088485 & -0.32535458 \\
\hline 1-C $\mathbf{L H}^{-}$ & -0.10216616 & -0.08857370 & -0.11803350 & -0.10050996 & -0.12455599 & -0.10506772 \\
\hline 4-C LH' $\mathbf{L H}^{-}$ & -0.09865184 & -0.08613545 & -0.11383352 & -0.09762549 & -0.11972831 & -0.10180079 \\
\hline EP & -0.02435849 & -0.01426294 & -0.02915763 & -0.01818326 & -0.03270623 & -0.02154581 \\
\hline $\mathbf{E P}^{2-}$ & -0.30878378 & -0.28758565 & -0.33535454 & -0.30817529 & -0.34435011 & -0.31431075 \\
\hline $1-\mathrm{C} \mathbf{E P}-$ & -0.10964675 & -0.09184063 & -0.12440748 & -0.10232669 & -0.13040017 & -0.10634262 \\
\hline 4-C EP- & -0.10274751 & -0.08702788 & -0.11598585 & -0.09671713 & -0.12108564 & -0.10027091 \\
\hline 1-C LHOOH & -0.02817934 & -0.01518725 & -0.03546407 & -0.02101992 & -0.04175174 & -0.02647011 \\
\hline 4-C LHOOH & -0.02813695 & -0.01461354 & -0.03522877 & -0.02035059 & -0.04177284 & -0.02596015 \\
\hline 1-C L-OOH & -0.10087775 & -0.08436653 & -0.11769745 & -0.09719521 & -0.12510465 & -0.10296414 \\
\hline 4-C L-OOH & -0.09363477 & -0.07880478 & -0.10844602 & -0.09029482 & -0.11523679 & -0.09571314 \\
\hline 1-C LHOO' & -0.11544688 & -0.09515537 & -0.13561928 & -0.10956175 & -0.14580130 & -0.11684462 \\
\hline 4-C LHOO' & -0.11025556 & -0.09137848 & -0.12941624 & -0.10535458 & -0.13924246 & -0.11258964 \\
\hline $1-\mathrm{C} \mathbf{P I}^{-}$ & -0.08476070 & -0.07235059 & -0.09625771 & -0.08157768 & -0.09942643 & -0.08395219 \\
\hline 4-C PI' & -0.07863022 & -0.06843027 & -0.08836563 & -0.07654183 & -0.09081945 & -0.07853386 \\
\hline $1-\mathrm{C} \mathbf{P I}^{2-}$ & -0.31791930 & -0.28901992 & -0.34555872 & -0.30972111 & -0.35744871 & -0.31725896 \\
\hline 4-C PI ${ }^{2-}$ & -0.32013584 & -0.28949800 & -0.34874868 & -0.31015139 & -0.36150306 & -0.31759362 \\
\hline
\end{tabular}




\begin{tabular}{|c|c|c|c|c|c|c|c|}
\hline \multicolumn{8}{|c|}{ PBE0DH/6-31+G* } \\
\hline & \multirow[b]{2}{*}{$G_{\text {gas }}\left(\mathrm{S}_{0}\right)$} & \multicolumn{2}{|c|}{ UAHF } & \multicolumn{2}{|c|}{ UAKS } & \multicolumn{2}{|c|}{ UFF } \\
\hline & & $\Delta G_{\text {pol.solu.-solv. }}$ & $\Delta G_{\text {solv }}$ & $\Delta G_{\text {pol.solu.-solv. }}$ & $\Delta G_{\text {solv }}$ & $\Delta G_{\text {pol.solu.-solv. }}$ & $\Delta G_{\text {solv }}$ \\
\hline $\mathbf{L H}_{2}$ & -622.92258600 & -0.06549597 & -0.04768127 & -0.07121451 & -0.05207968 & -0.02021630 & -0.01123505 \\
\hline $\mathbf{L}^{2-}$ & -621.69517200 & -0.36408998 & -0.32427091 & -0.37648091 & -0.33233466 & -0.32050250 & -0.29628685 \\
\hline 1-C $\mathbf{L} \mathbf{H}^{-}$ & -622.39566900 & -0.12364946 & -0.10320318 & -0.13464757 & -0.11164940 & -0.10011149 & -0.08648605 \\
\hline 4-C $\mathbf{L} \mathbf{H}^{-}$ & -622.39840300 & -0.11978336 & -0.10058964 & -0.13033658 & -0.10871713 & -0.09686030 & -0.08428685 \\
\hline EP & -773.03398700 & -0.05523658 & -0.04020717 & -0.05875941 & -0.04318725 & -0.01667409 & -0.00750597 \\
\hline $\mathbf{E P}^{2-}$ & -771.84532600 & -0.35021688 & -0.31730677 & -0.35438745 & -0.32046215 & -0.31001174 & -0.28892430 \\
\hline 1-C EP' & -772.50440200 & -0.14612069 & -0.11805577 & -0.15039309 & -0.12137051 & -0.10687752 & -0.08968924 \\
\hline 4-C EP- & -772.51295500 & -0.13576647 & -0.11118725 & -0.13921636 & -0.11397609 & -0.09993456 & -0.08489243 \\
\hline 1-C LHOOH & -773.03928600 & -0.06167927 & -0.04325099 & -0.06635976 & -0.04710756 & -0.02085160 & -0.00911553 \\
\hline 4-C LHOOH & -773.03260100 & -0.06159088 & -0.04226294 & -0.06595696 & -0.04576892 & -0.02099291 & -0.00847808 \\
\hline 1-C L-OOH & -772.51667100 & -0.13106342 & -0.10809561 & -0.13667953 & -0.11247808 & -0.10060967 & -0.08462151 \\
\hline 4-C L-OOH & -772.51960000 & -0.12072896 & -0.10027091 & -0.12513477 & -0.10374501 & -0.09368405 & -0.07917131 \\
\hline 1-C LHOO- & -772.48612800 & -0.14575886 & -0.11612749 & -0.15450508 & -0.12243824 & -0.10884939 & -0.08941832 \\
\hline 4-C LHOO- & -772.48421500 & -0.14298349 & -0.11550597 & -0.15275542 & -0.12293227 & -0.10629456 & -0.08776095 \\
\hline 1-C $\mathbf{P I}^{-}$ & -663.13972700 & -0.10713584 & -0.09019920 & -0.11518234 & -0.09643027 & -0.08562470 & -0.07345019 \\
\hline 4-C PI' & -663.14657200 & -0.09758437 & -0.08376095 & -0.10336885 & -0.08849402 & -0.08032374 & -0.07015139 \\
\hline 1-C $\mathbf{P I}^{2-}$ & -662.44852200 & -0.35398873 & -0.31306772 & -0.36581213 & -0.32189641 & -0.31791969 & -0.28881274 \\
\hline 4-C PI $\mathbf{P I}^{2-}$ & -662.45079700 & -0.34717681 & -0.30742629 & -0.35906147 & -0.31576095 & -0.31231337 & -0.28487649 \\
\hline
\end{tabular}




\begin{tabular}{|c|c|c|c|c|c|c|}
\hline \multicolumn{7}{|c|}{ PBE0DH/6-31+G* } \\
\hline & \multicolumn{2}{|c|}{ UA0 } & \multicolumn{2}{|c|}{ Bondi } & \multicolumn{2}{|c|}{ Pauling } \\
\hline & $\Delta G_{\text {pol.solu.-solv. }}$ & $\Delta G_{\text {solv }}$ & $\Delta G_{\text {pol.solu.-solv. }}$ & $\Delta G_{\text {solv. }}$ & $\Delta G_{\text {pol.solu.-solv. }}$ & $\Delta G_{\text {solv }}$ \\
\hline $\mathbf{L H}_{2}$ & -0.02592377 & -0.01611155 & -0.03533173 & -0.02294820 & -0.04096187 & -0.02683665 \\
\hline $\mathbf{L}^{2-}$ & -0.32094909 & -0.29558565 & -0.35400130 & -0.31921912 & -0.36360077 & -0.32511553 \\
\hline 1-C $\mathbf{L H}^{-}$ & -0.10239275 & -0.08811155 & -0.11885375 & -0.10003187 & -0.12530123 & -0.10411155 \\
\hline 4-C LH' & -0.09906406 & -0.08583266 & -0.11482752 & -0.09732270 & -0.12066047 & -0.10103585 \\
\hline EP & -0.02459721 & -0.01451792 & -0.02978013 & -0.01851792 & -0.03296744 & -0.02133864 \\
\hline $\mathbf{E P}^{2-}$ & -0.31019886 & -0.28833466 & -0.33814397 & -0.30949800 & -0.34774063 & -0.31560159 \\
\hline $1-\mathrm{C} \mathbf{E P}-$ & -0.11059069 & -0.09235059 & -0.12630643 & -0.10315537 & -0.13248976 & -0.10688446 \\
\hline 4-C EP- & -0.10329961 & -0.08728286 & -0.11729803 & -0.09716334 & -0.12250390 & -0.10041434 \\
\hline 1-C LHOOH & -0.02809432 & -0.01534661 & -0.03536923 & -0.02098804 & -0.04080562 & -0.02548207 \\
\hline 4-C LHOOH & -0.02826165 & -0.01467729 & -0.03541378 & -0.02020717 & -0.04112919 & -0.02486055 \\
\hline 1-C L-OOH & -0.10132959 & -0.08498804 & -0.11870309 & -0.09786454 & -0.12590106 & -0.10299601 \\
\hline 4-C L-OOH & -0.09411909 & -0.07933067 & -0.10946623 & -0.09085258 & -0.11604856 & -0.09568127 \\
\hline 1-C LHOO' & -0.11230977 & -0.09200000 & -0.13192755 & -0.10538645 & -0.14126986 & -0.11133067 \\
\hline 4-C LHOO' & -0.10941017 & -0.09015139 & -0.12928220 & -0.10404780 & -0.13911337 & -0.11061354 \\
\hline $1-\mathrm{C} \mathbf{P I}^{-}$ & -0.08584620 & -0.07329083 & -0.09813117 & -0.08290039 & -0.10152877 & -0.08513147 \\
\hline 4-C PI' & -0.07971935 & -0.06933864 & -0.09036564 & -0.07791235 & -0.09308964 & -0.07976095 \\
\hline $1-\mathrm{C} \mathbf{P I}^{2-}$ & -0.31882147 & -0.28850996 & -0.34816352 & -0.30981673 & -0.36184114 & -0.31759362 \\
\hline 4-C $\mathbf{P I}^{2-}$ & -0.31307774 & -0.28404780 & -0.34113649 & -0.30433466 & -0.35339102 & -0.31075697 \\
\hline
\end{tabular}


The optimized Cartesian coordinates $(\AA)$ of the stationary points and the imaginary frequencies of the transition states for the decomposition of $\mathrm{L}-\mathrm{OOH}$ in gas phase at PBE1PBE/6-31+G* level.

\begin{tabular}{|c|c|c|c|c|c|c|c|}
\hline \multicolumn{8}{|c|}{ TS1-1 } \\
\hline \multicolumn{4}{|c|}{$1-\mathrm{C}(228.2 i)$} & \multicolumn{4}{|c|}{ 4-C (321.0i) } \\
\hline $\mathrm{C}$ & -1.878 & 0.219 & -0.045 & $\mathrm{C}$ & -1.933 & -0.214 & 0.006 \\
\hline $\mathrm{C}$ & -0.483 & 0.175 & 0.078 & $\mathrm{C}$ & -0.515 & -0.183 & 0.153 \\
\hline $\mathrm{C}$ & 0.203 & -1.073 & 0.004 & $\mathrm{C}$ & 0.154 & 1.066 & 0.113 \\
\hline $\mathrm{C}$ & -0.560 & -2.280 & -0.034 & $\mathrm{C}$ & -0.578 & 2.267 & -0.010 \\
\hline $\mathrm{C}$ & -1.953 & -2.206 & -0.105 & $\mathrm{C}$ & -1.953 & 2.221 & -0.104 \\
\hline $\mathrm{C}$ & -2.596 & -0.963 & -0.142 & $\mathrm{C}$ & -2.625 & 0.990 & -0.113 \\
\hline $\mathrm{H}$ & -2.534 & -3.127 & -0.086 & $\mathrm{H}$ & -0.036 & 3.208 & -0.041 \\
\hline $\mathrm{H}$ & -3.683 & -0.932 & -0.194 & $\mathrm{H}$ & -2.529 & 3.143 & -0.160 \\
\hline $\mathrm{C}$ & 0.246 & 1.412 & 0.307 & $\mathrm{H}$ & -3.712 & 0.962 & -0.174 \\
\hline $\mathrm{C}$ & 1.559 & -1.143 & -0.483 & $\mathrm{C}$ & 0.252 & -1.407 & 0.294 \\
\hline $\mathrm{O}$ & 1.950 & 2.460 & 1.430 & $\mathrm{C}$ & 1.555 & 1.142 & -0.236 \\
\hline $\mathrm{O}$ & -0.200 & 2.568 & 0.186 & $\mathrm{O}$ & 2.027 & -2.460 & 1.270 \\
\hline $\mathrm{N}$ & 1.441 & 1.049 & -1.379 & $\mathrm{O}$ & -0.159 & -2.583 & 0.121 \\
\hline $\mathrm{N}$ & 1.988 & -0.010 & -1.472 & $\mathrm{~N}$ & 1.450 & -0.987 & -1.375 \\
\hline $\mathrm{O}$ & 1.298 & 1.207 & 1.216 & $\mathrm{~N}$ & 1.975 & 0.076 & -1.409 \\
\hline $\mathrm{O}$ & 2.381 & -2.030 & -0.334 & $\mathrm{~N}$ & -2.604 & -1.423 & 0.065 \\
\hline $\mathrm{H}$ & 1.250 & 3.050 & 1.037 & $\mathrm{H}$ & -3.457 & -1.455 & -0.471 \\
\hline $\mathrm{N}$ & 0.070 & -3.513 & 0.035 & $\mathrm{H}$ & -1.974 & -2.214 & -0.065 \\
\hline $\mathrm{H}$ & 1.081 & -3.467 & -0.073 & $\mathrm{O}$ & 1.302 & -1.230 & 1.205 \\
\hline $\mathrm{H}$ & -0.367 & -4.248 & -0.503 & $\mathrm{O}$ & 2.428 & 1.898 & 0.095 \\
\hline $\mathrm{H}$ & -2.370 & 1.187 & -0.015 & $\mathrm{H}$ & 1.321 & -3.037 & 0.877 \\
\hline
\end{tabular}




\begin{tabular}{|c|c|c|c|c|c|c|c|}
\hline \multicolumn{8}{|c|}{ TS1-2 } \\
\hline \multicolumn{4}{|c|}{$1-\mathrm{C}(248.9 i)$} & \multicolumn{4}{|c|}{ 4-C (235.3i) } \\
\hline $\mathrm{C}$ & -1.196 & -1.472 & 1.110 & $\mathrm{C}$ & -1.278 & 1.439 & 1.085 \\
\hline $\mathrm{C}$ & -0.417 & -0.531 & 0.351 & $\mathrm{C}$ & -0.433 & 0.501 & 0.361 \\
\hline $\mathrm{C}$ & -0.111 & -0.867 & -1.052 & $\mathrm{C}$ & -0.132 & 0.833 & -1.044 \\
\hline $\mathrm{C}$ & -0.462 & -2.188 & -1.557 & $\mathrm{C}$ & -0.402 & 2.173 & -1.538 \\
\hline $\mathrm{C}$ & -1.147 & -3.054 & -0.753 & $\mathrm{C}$ & -1.078 & 3.050 & -0.753 \\
\hline $\mathrm{C}$ & -1.537 & -2.678 & 0.585 & $\mathrm{C}$ & -1.561 & 2.667 & 0.548 \\
\hline $\mathrm{H}$ & -1.399 & -4.045 & -1.131 & $\mathrm{H}$ & -0.064 & 2.448 & -2.534 \\
\hline $\mathrm{H}$ & -2.119 & -3.384 & 1.174 & $\mathrm{H}$ & -1.276 & 4.057 & -1.115 \\
\hline $\mathrm{C}$ & 0.042 & 0.663 & 0.804 & $\mathrm{H}$ & -2.185 & 3.361 & 1.112 \\
\hline $\mathrm{C}$ & 0.423 & 0.012 & -1.917 & $\mathrm{C}$ & 0.104 & -0.651 & 0.848 \\
\hline $\mathrm{O}$ & -0.220 & 2.272 & -0.732 & $\mathrm{C}$ & 0.353 & -0.051 & -1.934 \\
\hline $\mathrm{O}$ & -0.230 & 1.146 & 2.054 & $\mathrm{O}$ & -0.061 & -2.290 & -0.625 \\
\hline $\mathrm{O}$ & 0.788 & 1.442 & 0.033 & $\mathrm{O}$ & -0.162 & -1.164 & 2.099 \\
\hline $\mathrm{O}$ & 0.923 & 0.517 & -2.850 & $\mathrm{~N}$ & -1.805 & 1.050 & 2.314 \\
\hline $\mathrm{H}$ & -0.172 & 2.109 & 1.936 & $\mathrm{H}$ & -1.978 & 1.812 & 2.954 \\
\hline $\mathrm{N}$ & -0.036 & -2.550 & -2.842 & $\mathrm{H}$ & -1.343 & 0.256 & 2.741 \\
\hline $\mathrm{H}$ & 0.046 & -1.775 & -3.491 & $\mathrm{O}$ & 0.909 & -1.399 & 0.114 \\
\hline $\mathrm{H}$ & -0.598 & -3.288 & -3.245 & $\mathrm{O}$ & 0.834 & -0.553 & -2.876 \\
\hline $\mathrm{H}$ & -1.489 & -1.192 & 2.118 & $\mathrm{H}$ & -0.140 & -2.125 & 1.943 \\
\hline
\end{tabular}




\begin{tabular}{|c|c|c|c|c|c|c|c|}
\hline \multicolumn{8}{|c|}{ TS1-3 } \\
\hline \multicolumn{4}{|c|}{$1-\mathrm{C}(202.3 i)$} & \multicolumn{4}{|c|}{ 4-C (198.5i) } \\
\hline $\mathrm{C}$ & -2.226 & -0.281 & -0.136 & $\mathrm{C}$ & -2.226 & 0.258 & -0.123 \\
\hline $\mathrm{C}$ & -0.826 & -0.221 & -0.098 & $\mathrm{C}$ & -0.813 & 0.184 & -0.052 \\
\hline $\mathrm{C}$ & -0.075 & -1.409 & 0.372 & $\mathrm{C}$ & -0.073 & 1.400 & 0.375 \\
\hline $\mathrm{C}$ & -0.859 & -2.615 & 0.657 & $\mathrm{C}$ & -0.847 & 2.613 & 0.663 \\
\hline $\mathrm{C}$ & -2.220 & -2.605 & 0.564 & $\mathrm{C}$ & -2.204 & 2.609 & 0.533 \\
\hline $\mathrm{C}$ & -2.952 & -1.417 & 0.190 & $\mathrm{C}$ & -2.933 & 1.441 & 0.133 \\
\hline $\mathrm{H}$ & -2.761 & -3.533 & 0.777 & $\mathrm{H}$ & -0.316 & 3.519 & 0.957 \\
\hline $\mathrm{H}$ & -4.040 & -1.438 & 0.130 & $\mathrm{H}$ & -2.754 & 3.530 & 0.746 \\
\hline $\mathrm{C}$ & -0.106 & 0.990 & -0.352 & $\mathrm{H}$ & -4.022 & 1.420 & 0.172 \\
\hline $\mathrm{C}$ & 1.247 & -1.442 & 0.571 & $\mathrm{C}$ & -0.071 & -0.996 & -0.360 \\
\hline $\mathrm{O}$ & 1.747 & 0.245 & -1.478 & $\mathrm{C}$ & 1.251 & 1.481 & 0.498 \\
\hline $\mathrm{O}$ & -0.610 & 2.112 & -0.557 & $\mathrm{O}$ & 1.775 & -0.251 & -1.472 \\
\hline $\mathrm{O}$ & 1.252 & 0.858 & -0.210 & $\mathrm{O}$ & -0.558 & -2.121 & -0.656 \\
\hline $\mathrm{O}$ & 2.377 & -1.779 & 0.770 & $\mathrm{~N}$ & -2.946 & -0.909 & -0.427 \\
\hline $\mathrm{N}$ & -0.144 & -3.777 & 1.075 & $\mathrm{H}$ & -3.701 & -0.725 & -1.078 \\
\hline $\mathrm{H}$ & 0.759 & -3.825 & 0.608 & $\mathrm{H}$ & -2.296 & -1.641 & -0.741 \\
\hline $\mathrm{H}$ & -0.669 & -4.613 & 0.838 & $\mathrm{O}$ & 1.276 & -0.870 & -0.203 \\
\hline $\mathrm{H}$ & -2.733 & 0.632 & -0.445 & $\mathrm{O}$ & 2.367 & 1.836 & 0.719 \\
\hline
\end{tabular}




\begin{tabular}{|c|c|c|c|c|c|c|c|}
\hline \multicolumn{8}{|c|}{ TS1-4 } \\
\hline \multicolumn{4}{|c|}{$1-\mathrm{C}(412.7 i)$} & \multicolumn{4}{|c|}{$4-\mathrm{C}(424.5 i)$} \\
\hline $\mathrm{C}$ & -1.876 & 0.222 & -0.033 & $\mathrm{C}$ & -1.927 & -0.230 & 0.039 \\
\hline $\mathrm{C}$ & -0.485 & 0.155 & 0.192 & $\mathrm{C}$ & -0.510 & -0.176 & 0.249 \\
\hline $\mathrm{C}$ & 0.182 & -1.111 & 0.138 & $\mathrm{C}$ & 0.135 & 1.097 & 0.204 \\
\hline $\mathrm{C}$ & -0.573 & -2.314 & -0.093 & $\mathrm{C}$ & -0.590 & 2.289 & -0.053 \\
\hline $\mathrm{C}$ & -1.945 & -2.201 & -0.270 & $\mathrm{C}$ & -1.951 & 2.201 & -0.233 \\
\hline $\mathrm{C}$ & -2.575 & -0.945 & -0.257 & $\mathrm{C}$ & -2.607 & 0.960 & -0.193 \\
\hline $\mathrm{H}$ & -2.542 & -3.104 & -0.369 & $\mathrm{H}$ & -0.069 & 3.240 & -0.080 \\
\hline $\mathrm{H}$ & -3.652 & -0.901 & -0.399 & $\mathrm{H}$ & -2.537 & 3.105 & -0.379 \\
\hline $\mathrm{C}$ & 0.307 & 1.308 & 0.360 & $\mathrm{H}$ & -3.688 & 0.925 & -0.314 \\
\hline $\mathrm{C}$ & 1.566 & -1.182 & -0.078 & $\mathrm{C}$ & 0.335 & -1.308 & 0.353 \\
\hline $\mathrm{O}$ & 2.368 & 2.132 & 0.920 & $\mathrm{C}$ & 1.535 & 1.181 & 0.065 \\
\hline $\mathrm{O}$ & -0.244 & 2.534 & 0.350 & $\mathrm{O}$ & 2.450 & -1.993 & 0.944 \\
\hline $\mathrm{N}$ & 1.380 & 1.188 & -1.381 & $\mathrm{O}$ & -0.123 & -2.584 & 0.271 \\
\hline $\mathrm{N}$ & 1.912 & 0.181 & -1.508 & $\mathrm{~N}$ & 1.403 & -1.133 & -1.326 \\
\hline $\mathrm{O}$ & 1.378 & 1.104 & 1.188 & $\mathrm{~N}$ & 1.894 & -0.102 & -1.446 \\
\hline $\mathrm{O}$ & 2.540 & -1.815 & 0.124 & $\mathrm{~N}$ & -2.612 & -1.416 & 0.154 \\
\hline $\mathrm{H}$ & 2.532 & 2.427 & 1.831 & $\mathrm{H}$ & -3.535 & -1.442 & -0.254 \\
\hline $\mathrm{H}$ & 0.434 & 3.157 & 0.039 & $\mathrm{H}$ & -2.078 & -2.269 & 0.060 \\
\hline $\mathrm{N}$ & 0.046 & -3.539 & -0.063 & $\mathrm{O}$ & 1.362 & -1.088 & 1.237 \\
\hline $\mathrm{H}$ & 1.056 & -3.577 & -0.073 & $\mathrm{O}$ & 2.517 & 1.748 & 0.360 \\
\hline $\mathrm{H}$ & -0.405 & -4.296 & -0.553 & $\mathrm{H}$ & 2.515 & -2.446 & 1.801 \\
\hline $\mathrm{H}$ & -2.373 & 1.185 & 0.001 & $\mathrm{H}$ & 0.471 & -3.059 & -0.337 \\
\hline
\end{tabular}




\begin{tabular}{|c|c|c|c|c|c|c|c|}
\hline \multicolumn{8}{|c|}{ TS2-1 } \\
\hline \multicolumn{4}{|c|}{$1-\mathrm{C}(116.1 i)$} & \multicolumn{4}{|c|}{$4-\mathrm{C}(122.9 i)$} \\
\hline $\mathrm{C}$ & -1.334 & -1.056 & 0.934 & $\mathrm{C}$ & -1.355 & -1.044 & -0.959 \\
\hline $\mathrm{C}$ & -0.484 & -0.257 & 0.181 & $\mathrm{C}$ & -0.502 & -0.220 & -0.197 \\
\hline $\mathrm{C}$ & -0.016 & -0.662 & -1.076 & $\mathrm{C}$ & -0.054 & -0.647 & 1.052 \\
\hline $\mathrm{C}$ & -0.382 & -1.934 & -1.580 & $\mathrm{C}$ & -0.438 & -1.882 & 1.578 \\
\hline $\mathrm{C}$ & -1.245 & -2.736 & -0.808 & $\mathrm{C}$ & -1.285 & -2.694 & 0.833 \\
\hline $\mathrm{C}$ & -1.716 & -2.297 & 0.420 & $\mathrm{C}$ & -1.737 & -2.280 & -0.417 \\
\hline $\mathrm{H}$ & -1.525 & -3.719 & -1.186 & $\mathrm{H}$ & -1.599 & -3.661 & 1.222 \\
\hline $\mathrm{H}$ & -2.382 & -2.939 & 0.994 & $\mathrm{H}$ & -2.391 & -2.929 & -1.001 \\
\hline $\mathrm{C}$ & 0.065 & 1.034 & 0.709 & $\mathrm{C}$ & 0.060 & 1.083 & -0.689 \\
\hline $\mathrm{C}$ & 0.743 & 0.315 & -1.887 & $\mathrm{C}$ & 0.802 & 0.264 & 1.868 \\
\hline $\mathrm{O}$ & 2.097 & 1.815 & 0.894 & $\mathrm{O}$ & 2.112 & 1.807 & -0.946 \\
\hline $\mathrm{O}$ & -0.931 & 1.686 & 1.448 & $\mathrm{O}$ & -0.973 & 1.820 & -1.321 \\
\hline $\mathrm{N}$ & 0.501 & 2.032 & -0.326 & $\mathrm{~N}$ & 0.564 & 2.026 & 0.367 \\
\hline $\mathrm{N}$ & 0.752 & 1.671 & -1.517 & $\mathrm{~N}$ & 0.847 & 1.633 & 1.536 \\
\hline $\mathrm{O}$ & 1.209 & 0.787 & 1.430 & $\mathrm{O}$ & 1.139 & 0.869 & -1.502 \\
\hline $\mathrm{O}$ & 1.246 & 0.007 & -2.974 & $\mathrm{O}$ & 1.346 & -0.128 & 2.890 \\
\hline $\mathrm{H}$ & -0.544 & 2.556 & 1.641 & $\mathrm{H}$ & -0.585 & 2.705 & -1.433 \\
\hline $\mathrm{N}$ & 0.117 & -2.404 & -2.772 & $\mathrm{H}$ & -0.068 & -2.172 & 2.557 \\
\hline $\mathrm{H}$ & 0.604 & -1.694 & -3.320 & $\mathrm{~N}$ & -1.765 & -0.684 & -2.237 \\
\hline $\mathrm{H}$ & -0.459 & -3.061 & -3.276 & $\mathrm{H}$ & -2.658 & -1.070 & -2.511 \\
\hline $\mathrm{H}$ & -1.685 & -0.708 & 1.900 & $\mathrm{H}$ & -1.700 & 0.315 & -2.411 \\
\hline
\end{tabular}




\begin{tabular}{|c|c|c|c|c|c|c|c|}
\hline \multicolumn{8}{|c|}{ TS2-2 } \\
\hline \multicolumn{4}{|c|}{$1-\mathrm{C}(175.9 i)$} & \multicolumn{4}{|c|}{ 4-C (146.6i) } \\
\hline $\mathrm{C}$ & 13.923 & -14.936 & 13.201 & $\mathrm{C}$ & 13.977 & 14.978 & 13.251 \\
\hline $\mathrm{C}$ & 14.652 & -14.063 & 12.398 & $\mathrm{C}$ & 14.691 & 14.075 & 12.428 \\
\hline $\mathrm{C}$ & 14.932 & -14.382 & 11.060 & $\mathrm{C}$ & 14.965 & 14.421 & 11.105 \\
\hline $\mathrm{C}$ & 14.514 & -15.637 & 10.525 & $\mathrm{C}$ & 14.524 & 15.635 & 10.554 \\
\hline $\mathrm{C}$ & 13.789 & -16.504 & 11.351 & $\mathrm{C}$ & 13.776 & 16.489 & 11.344 \\
\hline $\mathrm{C}$ & 13.503 & -16.148 & 12.669 & $\mathrm{C}$ & 13.516 & 16.167 & 12.680 \\
\hline $\mathrm{H}$ & 13.462 & -17.465 & 10.956 & $\mathrm{H}$ & 14.771 & 15.866 & 9.522 \\
\hline $\mathrm{H}$ & 12.945 & -16.845 & 13.292 & $\mathrm{H}$ & 13.406 & 17.429 & 10.939 \\
\hline $\mathrm{C}$ & 15.001 & -12.697 & 12.922 & $\mathrm{H}$ & 12.976 & 16.869 & 13.314 \\
\hline $\mathrm{C}$ & 15.658 & -13.419 & 10.264 & $\mathrm{C}$ & 14.994 & 12.674 & 12.881 \\
\hline $\mathrm{O}$ & 16.677 & -13.765 & 13.803 & $\mathrm{C}$ & 15.760 & 13.505 & 10.286 \\
\hline $\mathrm{O}$ & 13.824 & -11.931 & 13.045 & $\mathrm{O}$ & 16.624 & 13.645 & 14.026 \\
\hline $\mathrm{N}$ & 15.840 & -11.850 & 12.011 & $\mathrm{O}$ & 13.797 & 11.927 & 12.900 \\
\hline $\mathrm{N}$ & 16.123 & -12.165 & 10.849 & $\mathrm{~N}$ & 15.860 & 11.861 & 11.963 \\
\hline $\mathrm{O}$ & 15.636 & -12.774 & 14.113 & $\mathrm{~N}$ & 16.218 & 12.229 & 10.838 \\
\hline $\mathrm{O}$ & 15.924 & -13.549 & 9.069 & $\mathrm{~N}$ & 13.783 & 14.727 & 14.600 \\
\hline $\mathrm{H}$ & 14.128 & -11.065 & 13.364 & $\mathrm{H}$ & 12.838 & 14.851 & 14.935 \\
\hline $\mathrm{N}$ & 14.843 & -16.001 & 9.243 & $\mathrm{H}$ & 14.227 & 13.865 & 14.908 \\
\hline $\mathrm{H}$ & 15.197 & -15.250 & 8.657 & $\mathrm{O}$ & 15.572 & 12.628 & 14.112 \\
\hline $\mathrm{H}$ & 14.275 & -16.709 & 8.805 & $\mathrm{O}$ & 16.065 & 13.713 & 9.126 \\
\hline $\mathrm{H}$ & 13.730 & -14.663 & 14.233 & $\mathrm{H}$ & 14.075 & 11.026 & 13.134 \\
\hline
\end{tabular}




\begin{tabular}{|c|c|c|c|c|c|c|c|}
\hline \multicolumn{8}{|c|}{ TS2-3 } \\
\hline \multicolumn{4}{|c|}{$1-\mathrm{C}(310.6 i)$} & \multicolumn{4}{|c|}{$4-\mathrm{C}(277.7 i)$} \\
\hline $\mathrm{C}$ & 13.666 & 14.918 & 13.214 & $\mathrm{C}$ & 13.652 & 14.895 & 13.206 \\
\hline $\mathrm{C}$ & 14.715 & 14.152 & 12.648 & $\mathrm{C}$ & 14.674 & 14.131 & 12.608 \\
\hline $\mathrm{C}$ & 15.292 & 14.560 & 11.432 & $\mathrm{C}$ & 15.243 & 14.539 & 11.388 \\
\hline $\mathrm{C}$ & 14.839 & 15.694 & 10.747 & $\mathrm{C}$ & 14.819 & 15.712 & 10.757 \\
\hline $\mathrm{C}$ & 13.831 & 16.452 & 11.326 & $\mathrm{C}$ & 13.836 & 16.480 & 11.364 \\
\hline $\mathrm{C}$ & 13.254 & 16.076 & 12.545 & $\mathrm{C}$ & 13.262 & 16.082 & 12.577 \\
\hline $\mathrm{H}$ & 15.283 & 15.962 & 9.793 & $\mathrm{H}$ & 15.272 & 15.989 & 9.809 \\
\hline $\mathrm{H}$ & 13.482 & 17.359 & 10.833 & $\mathrm{H}$ & 13.509 & 17.411 & 10.905 \\
\hline $\mathrm{H}$ & 12.479 & 16.696 & 12.995 & $\mathrm{H}$ & 12.512 & 16.709 & 13.058 \\
\hline $\mathrm{C}$ & 15.109 & 12.838 & 13.181 & $\mathrm{C}$ & 15.120 & 12.783 & 12.956 \\
\hline $\mathrm{C}$ & 16.185 & 13.549 & 10.901 & $\mathrm{C}$ & 16.199 & 13.627 & 10.725 \\
\hline $\mathrm{O}$ & 17.141 & 13.109 & 11.869 & $\mathrm{O}$ & 17.093 & 12.936 & 11.635 \\
\hline $\mathrm{O}$ & 14.608 & 12.276 & 14.167 & $\mathrm{O}$ & 14.670 & 12.234 & 14.134 \\
\hline $\mathrm{N}$ & 14.677 & 11.812 & 11.492 & $\mathrm{~N}$ & 14.684 & 11.765 & 11.832 \\
\hline $\mathrm{N}$ & 15.349 & 12.219 & 10.591 & $\mathrm{~N}$ & 14.988 & 12.069 & 10.711 \\
\hline $\mathrm{N}$ & 13.113 & 14.552 & 14.431 & $\mathrm{~N}$ & 13.085 & 14.532 & 14.432 \\
\hline $\mathrm{H}$ & 12.137 & 14.790 & 14.541 & $\mathrm{H}$ & 12.105 & 14.768 & 14.511 \\
\hline $\mathrm{H}$ & 13.343 & 13.585 & 14.674 & $\mathrm{H}$ & 13.273 & 13.568 & 14.683 \\
\hline $\mathrm{O}$ & 16.491 & 12.496 & 13.019 & $\mathrm{O}$ & 16.542 & 12.681 & 12.962 \\
\hline $\mathrm{O}$ & 16.865 & 13.845 & 9.755 & $\mathrm{O}$ & 16.714 & 13.812 & 9.624 \\
\hline $\mathrm{H}$ & 16.836 & 13.015 & 9.238 & $\mathrm{H}$ & 14.509 & 11.296 & 13.901 \\
\hline
\end{tabular}




\begin{tabular}{|c|c|c|c|c|c|c|c|}
\hline \multicolumn{4}{|c|}{ TS2-4 (383.5i) } & \multicolumn{4}{|c|}{ TS2-5 (473.8i) } \\
\hline $\mathrm{C}$ & 15.283 & 12.943 & 13.265 & $\mathrm{C}$ & 13.650 & 14.902 & 13.229 \\
\hline $\mathrm{C}$ & 14.523 & 14.127 & 13.398 & $\mathrm{C}$ & 14.738 & 14.155 & 12.664 \\
\hline $\mathrm{C}$ & 15.178 & 15.379 & 13.414 & $\mathrm{C}$ & 15.299 & 14.573 & 11.417 \\
\hline $\mathrm{C}$ & 16.577 & 15.453 & 13.309 & $\mathrm{C}$ & 14.800 & 15.709 & 10.738 \\
\hline $\mathrm{C}$ & 17.312 & 14.288 & 13.141 & $\mathrm{C}$ & 13.790 & 16.429 & 11.328 \\
\hline $\mathrm{C}$ & 16.670 & 13.035 & 13.108 & $\mathrm{C}$ & 13.223 & 16.036 & 12.558 \\
\hline $\mathrm{H}$ & 17.045 & 16.434 & 13.360 & $\mathrm{H}$ & 15.235 & 16.000 & 9.788 \\
\hline $\mathrm{H}$ & 18.396 & 14.337 & 13.014 & $\mathrm{H}$ & 13.419 & 17.330 & 10.848 \\
\hline $\mathrm{H}$ & 17.245 & 12.123 & 12.929 & $\mathrm{H}$ & 12.439 & 16.645 & 13.003 \\
\hline $\mathrm{C}$ & 13.102 & 14.143 & 13.800 & $\mathrm{C}$ & 15.135 & 12.867 & 13.055 \\
\hline $\mathrm{C}$ & 14.365 & 16.551 & 13.764 & $\mathrm{C}$ & 16.151 & 13.635 & 10.814 \\
\hline $\mathrm{O}$ & 13.040 & 16.527 & 13.154 & $\mathrm{O}$ & 17.022 & 12.864 & 11.580 \\
\hline $\mathrm{O}$ & 12.383 & 13.082 & 13.838 & $\mathrm{O}$ & 14.668 & 12.247 & 14.154 \\
\hline $\mathrm{N}$ & 13.196 & 14.901 & 15.367 & $\mathrm{~N}$ & 14.448 & 11.761 & 11.550 \\
\hline $\mathrm{N}$ & 13.774 & 15.945 & 15.403 & $\mathrm{~N}$ & 14.887 & 12.116 & 10.547 \\
\hline $\mathrm{N}$ & 14.627 & 11.698 & 13.232 & $\mathrm{~N}$ & 13.118 & 14.552 & 14.450 \\
\hline $\mathrm{H}$ & 15.078 & 11.020 & 13.836 & $\mathrm{H}$ & 12.213 & 14.940 & 14.670 \\
\hline $\mathrm{H}$ & 13.642 & 11.866 & 13.514 & $\mathrm{H}$ & 13.258 & 13.603 & 14.764 \\
\hline $\mathrm{O}$ & 12.371 & 15.217 & 13.130 & $\mathrm{O}$ & 16.453 & 12.450 & 12.860 \\
\hline \multirow[t]{3}{*}{$\mathrm{O}$} & 14.812 & 17.726 & 13.827 & $\mathrm{O}$ & 16.703 & 13.852 & 9.617 \\
\hline & & & & $\mathrm{H}$ & 14.668 & 11.289 & 13.982 \\
\hline & & & & $\mathrm{H}$ & 16.902 & 12.994 & 9.204 \\
\hline
\end{tabular}




\begin{tabular}{|c|c|c|c|c|c|c|c|}
\hline \multicolumn{4}{|c|}{$\mathbf{C P}$} & \multicolumn{4}{|c|}{$\mathrm{TS}_{\mathrm{O}-\mathrm{O}}^{\prime}(303.5 i)$} \\
\hline $\mathrm{C}$ & -1.884 & 0.202 & 0.137 & $\mathrm{C}$ & -1.850 & 0.139 & 0.170 \\
\hline $\mathrm{C}$ & -0.508 & -0.222 & -0.089 & $\mathrm{C}$ & -0.442 & -0.237 & 0.105 \\
\hline $\mathrm{C}$ & 0.535 & 0.815 & -0.193 & $\mathrm{C}$ & 0.578 & 0.830 & 0.049 \\
\hline $\mathrm{C}$ & 0.138 & 2.189 & -0.395 & $\mathrm{C}$ & 0.146 & 2.173 & -0.227 \\
\hline $\mathrm{C}$ & -1.176 & 2.512 & -0.280 & $\mathrm{C}$ & -1.184 & 2.456 & -0.235 \\
\hline $\mathrm{C}$ & -2.182 & 1.533 & 0.025 & $\mathrm{C}$ & -2.183 & 1.456 & -0.007 \\
\hline $\mathrm{H}$ & 0.881 & 2.945 & -0.636 & $\mathrm{H}$ & 0.876 & 2.972 & -0.339 \\
\hline $\mathrm{H}$ & -1.490 & 3.544 & -0.418 & $\mathrm{H}$ & -1.512 & 3.481 & -0.394 \\
\hline $\mathrm{H}$ & -3.212 & 1.858 & 0.149 & $\mathrm{H}$ & -3.230 & 1.750 & 0.026 \\
\hline $\mathrm{C}$ & -0.072 & -1.489 & -0.241 & $\mathrm{C}$ & 0.009 & -1.493 & -0.133 \\
\hline $\mathrm{C}$ & 1.800 & 0.400 & 0.039 & $\mathrm{C}$ & 1.861 & 0.411 & 0.183 \\
\hline $\mathrm{O}$ & 2.063 & -0.924 & 0.318 & $\mathrm{O}$ & 2.071 & -0.864 & 0.614 \\
\hline $\mathrm{O}$ & -0.805 & -2.619 & -0.322 & $\mathrm{O}$ & -0.736 & -2.543 & -0.517 \\
\hline $\mathrm{N}$ & -2.825 & -0.751 & 0.472 & $\mathrm{~N}$ & -2.792 & -0.828 & 0.454 \\
\hline $\mathrm{H}$ & -3.789 & -0.477 & 0.348 & $\mathrm{H}$ & -3.750 & -0.564 & 0.268 \\
\hline $\mathrm{H}$ & -2.632 & -1.702 & 0.189 & $\mathrm{H}$ & -2.576 & -1.771 & 0.164 \\
\hline $\mathrm{O}$ & 1.253 & -1.713 & -0.561 & $\mathrm{O}$ & 1.339 & -1.790 & -0.268 \\
\hline $\mathrm{O}$ & 2.920 & 1.114 & 0.214 & $\mathrm{O}$ & 3.022 & 1.067 & -0.029 \\
\hline $\mathrm{H}$ & -0.264 & -3.367 & -0.021 & $\mathrm{H}$ & -0.263 & -3.362 & -0.297 \\
\hline $\mathrm{H}$ & 2.679 & 2.005 & 0.508 & $\mathrm{H}$ & 2.830 & 1.923 & -0.438 \\
\hline
\end{tabular}




\begin{tabular}{|c|c|c|c|c|c|c|c|}
\hline \multicolumn{4}{|c|}{$\mathbf{C P}^{2-}$} & \multicolumn{4}{|c|}{$\mathrm{TS}_{\mathrm{O}-\mathrm{O}}(1736.7 i)$} \\
\hline $\mathrm{C}$ & -1.852 & 0.187 & 0.135 & $\mathrm{C}$ & -1.781 & 0.143 & 0.160 \\
\hline $\mathrm{C}$ & -0.478 & -0.232 & 0.042 & $\mathrm{C}$ & -0.405 & -0.265 & 0.120 \\
\hline $\mathrm{C}$ & 0.567 & 0.824 & -0.063 & $\mathrm{C}$ & 0.626 & 0.800 & 0.024 \\
\hline $\mathrm{C}$ & 0.141 & 2.179 & -0.241 & $\mathrm{C}$ & 0.197 & 2.140 & -0.214 \\
\hline $\mathrm{C}$ & -1.184 & 2.526 & -0.215 & $\mathrm{C}$ & -1.133 & 2.481 & -0.207 \\
\hline $\mathrm{C}$ & -2.206 & 1.514 & 0.004 & $\mathrm{C}$ & -2.144 & 1.473 & 0.026 \\
\hline $\mathrm{H}$ & 0.913 & 2.940 & -0.361 & $\mathrm{H}$ & 0.970 & 2.895 & -0.360 \\
\hline $\mathrm{H}$ & -1.484 & 3.569 & -0.335 & $\mathrm{H}$ & -1.437 & 3.519 & -0.360 \\
\hline $\mathrm{H}$ & -3.252 & 1.804 & 0.131 & $\mathrm{H}$ & -3.193 & 1.755 & 0.140 \\
\hline $\mathrm{C}$ & -0.122 & -1.562 & -0.136 & $\mathrm{C}$ & -0.049 & -1.602 & -0.135 \\
\hline $\mathrm{C}$ & 1.901 & 0.491 & 0.115 & $\mathrm{C}$ & 1.976 & 0.460 & 0.128 \\
\hline $\mathrm{O}$ & 2.110 & -0.903 & 0.399 & $\mathrm{O}$ & 2.168 & -0.875 & 0.502 \\
\hline $\mathrm{O}$ & -0.812 & -2.622 & -0.251 & $\mathrm{O}$ & -0.789 & -2.604 & -0.374 \\
\hline $\mathrm{N}$ & -2.825 & -0.808 & 0.374 & $\mathrm{~N}$ & -2.770 & -0.846 & 0.359 \\
\hline $\mathrm{H}$ & -3.675 & -0.616 & -0.144 & $\mathrm{H}$ & -3.607 & -0.622 & -0.170 \\
\hline $\mathrm{H}$ & -2.433 & -1.722 & 0.120 & $\mathrm{H}$ & -2.387 & -1.751 & 0.068 \\
\hline $\mathrm{O}$ & 1.258 & -1.770 & -0.390 & $\mathrm{O}$ & 1.297 & -1.889 & -0.297 \\
\hline $\mathrm{O}$ & 2.954 & 1.173 & 0.194 & $\mathrm{O}$ & 3.028 & 1.153 & -0.008 \\
\hline
\end{tabular}


Absolute free energies of $S_{0}$ state in gas phase and solvation energies of the optimized stationary points at PBE1PBE/6-31+G* levels. (unit: Hartree)

\begin{tabular}{|c|c|c|c|c|c|c|}
\hline & \multicolumn{2}{|c|}{$G_{\text {gas }}\left(\mathrm{S}_{0}\right)$} & \multicolumn{2}{|c|}{$\Delta G_{\text {pol.solu.-solv }}$} & \multicolumn{2}{|c|}{$\Delta G_{\text {solv. }}$} \\
\hline & $1-\mathrm{C}$ & $4-\mathrm{C}$ & $1-\mathrm{C}$ & $4-\mathrm{C}$ & $1-\mathrm{C}$ & $4-\mathrm{C}$ \\
\hline TS1-1 & -772.59476500 & -772.59619500 & -0.10771401 & -0.10274687 & -0.08642231 & -0.08296414 \\
\hline TS1-2 & -663.17113500 & -663.17252100 & -0.12358976 & -0.11759398 & -0.10145020 & -0.09735458 \\
\hline TS1-3 & -662.50512500 & -662.51546700 & -0.35089968 & -0.33851099 & -0.31537849 & -0.30755378 \\
\hline TS1-4 & -773.09068200 & -773.08783000 & -0.02930319 & -0.02869233 & -0.01464542 & -0.01376892 \\
\hline TS2-1 & -772.57418900 & -772.57006300 & -0.12504114 & -0.12340889 & -0.10129084 & -0.10047809 \\
\hline TS2-2 & -772.57511800 & -772.57321100 & -0.13617555 & -0.12862050 & -0.10919522 & -0.10406375 \\
\hline TS2-3 & -772.59701800 & -772.59037000 & -0.10734619 & -0.11506512 & -0.08894024 & -0.09399203 \\
\hline TS2-4 & \multicolumn{2}{|c|}{-771.93413600} & \multicolumn{2}{|c|}{-0.32872476} & \multicolumn{2}{|c|}{-0.30068526} \\
\hline TS2-5 & \multicolumn{2}{|c|}{-773.09155200} & \multicolumn{2}{|c|}{-0.02567150} & \multicolumn{2}{|c|}{-0.01403984} \\
\hline $\mathbf{C P}$ & \multicolumn{2}{|c|}{-663.73322700} & \multicolumn{2}{|c|}{-0.02848128} & \multicolumn{2}{|c|}{-0.01783267} \\
\hline $\mathrm{TS}_{\mathrm{O}-\mathrm{O}}^{\prime}$ & \multicolumn{2}{|c|}{-663.73165200} & \multicolumn{2}{|c|}{-0.03093069} & \multicolumn{2}{|c|}{-0.01974502} \\
\hline $\mathbf{C} \mathbf{P}^{2-}$ & \multicolumn{2}{|c|}{-662.58316800} & \multicolumn{2}{|c|}{-0.31467463} & \multicolumn{2}{|c|}{-0.29303586} \\
\hline $\mathrm{TS}_{\mathrm{O}-\mathrm{O}}$ & \multicolumn{2}{|c|}{-662.58106800} & \multicolumn{2}{|c|}{-0.32253074} & \multicolumn{2}{|c|}{-0.29534661} \\
\hline
\end{tabular}




\section{References}

(1) Isacsson, U.; Kowalewska, J.; Wettermark, G. J. Inorg. Nucl. Chem. 1978, 40, 1653.

(2) Khan, P.; Idrees, D.; Moxley, M. A.; Corbett, J. A.; Ahmad, F.; von Figura, G.; Sly, W. S.; Waheed, A.; Hassan, M. I. Appl. Biochem. Biotechnol. 2014, 173, 333. 